\title{
NORMALLY FLAT DEFORMATIONS
}

\author{
BY
}

B. M. BENNETT(1)

\begin{abstract}
We study flat families $Z / T$, together with a section $\sigma: T \rightarrow Z$ such that the normal cone to the image of $\sigma$ in $Z$ is flat over $T$. Such a family is called a "normally flat deformation (along $\sigma$ )"; it corresponds intuitively to a deformation of a singularity which preserves the Hilbert-Samuel function. We construct the versal normally flat deformation of an isolated singularity $(X, x)$ in terms of the flat strata of the relative jets of the "usual" versal deformation of $X$. We give explicit criteria, in terms of equations, for a fiat family to be normally flat along a given section. These criteria are applied to demonstrate the smoothness of normally flat deformation theoryand of the canonical map from it to the cone deformation theory of the tangent cone-in the case of strict complete intersections. Finally we study the tangent space to the normally flat deformation theory, expressing it as the sum of two spaces: The first is a piece of a certain filtration of the tangent space to the usual deformation theory of $X$; the second is the tangent space to the special fibre of the canonical map $N \rightarrow S$, where $N$ (resp. $S$ ) is the parameter space for the versal normally flat deformation of $(X, x)$ (resp. for the versal deformation of $X$ ). We discuss the relation of this second space to infinitesimal properties of sections.
\end{abstract}

Introduction. A basic numerical invariant of a singularity is its HilbertSamuel function (see (1.2.1) for a definition): equivalent to the multiplicity in the case of a hypersurface (but not otherwise), it provides one important index for the progress of desingularization procedures. Moreover it gives a good stratification of singular loci (on excellent schemes [6]); these strata should be strictly coarser than equisingular strata. Now if one thinks of a Hilbert-Samuel stratum $T$ in the singular locus of some variety $Z$ as the parameter space for a family of suitable transversal sections to $T$ in $Z$, one comes to the idea of a normally flat deformation. Thus, intuitively, a normally flat deformation of a singularity is one which carries along the singular point in such a way that the Hilbert-Samuel function is preserved; if one weakens this to require only that the first $j$ values are preserved, one obtains the concept of a normally flat deformation of order $j$.

In attempting to understand this as an infinitesimal deformation theory-

Received by the editors October 31, 1974.

AMS (MOS) subject classifications (1970). Primary 14B05, 14B10, $14 \mathrm{D} 15$.

(1) Sponsored by NSF GP-33045. 
following the approach of Schlessinger [4]-one is led to a formulation in which the basic object is: a (flat) family $Z \rightarrow T$ (i.e. an "ordinary" deformation) together with a section $\sigma: T \rightarrow Z$ such that the normal cone to the image of $\sigma$ in $Z$ is flat over $T$. From this point of view it is seen immediately that the normally flat deformation theory of a singularity $(X, x)$ is the right one to map functorially into the deformation theory of (i) the tangent cone to $X$ at $x$ and (ii) the blowing up of $X$ at $x$. The normally flat deformations of order $j$ correspond to families with sections whose normal cone is flat through degree $j$; equivalently this can be viewed as a deformation of $X$ which induces a normally flat deformation of the $j$ th infinitesimal neighborhood of $x$ in $X$.

Thus, given $(X, x)$ we define the functor $\pi$ (resp. $\varkappa_{j}$ ) of normally flat deformations (resp. normally flat deformations of order $j$ ) of $X$ at $x$; it is not hard to see that $\Re$ and $\Re_{j}$ coincide for $j$ sufficiently large. These assign to a scheme $T$ a set of pairs $(Z, \sigma)$ as above, modulo $T$-isomorphism. If we restrict ourselves to artin schemes $T$, these functors have "prorepresentable hulls" $N_{j}$ in the sense of [4]: If $\mathfrak{X} / S$ is the versal deformation of $X$ (so that $S$ is the space of "formal moduli"), $N_{j}$ embeds canonically as a subscheme of $\mathfrak{X}$ (not of $S$ ); in fact the $N_{j}$ are the intersections of the flat strata (or more precisely, their components through the point $x$ in the special fibre of $\mathfrak{X}$ ) of the sheaves of "principal parts of order $n$ " $P_{\mathfrak{X} / S}^{n}$ for $n \leqslant j$. The versal normally flat deformation of order $j$ is then

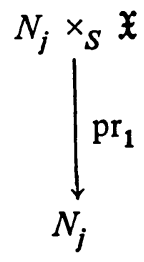

with versal section $\left(1_{N_{j}}, i\right)$, where $i: N_{j} \hookrightarrow \mathfrak{X}$ is the embedding. This is exposed in $\S 1$. We note that Lejeune and Teissier [7], [9] have observed that relative Hilbert-Samuel strata of a morphism may be defined, and may be used in the case of $\mathfrak{X} / \mathrm{S}$ to give a versal equimultiple family in a suitable sense.

Now in order to progress beyong the "abstract" existence theorems of $\S 1$, the first basic problem is to give explicit conditions for a deformation of a fixed singularity to be normally flat of order $j$ along a given section. In general, if $T \hookrightarrow Z$ is a subscheme, Hironaka [8] gives necessary and sufficient conditions for the normal cone to $T$ in $Z$ to be flat over $T$ at a point-provided $T$ is nonsingular there; in fact the terminology "normal flatness of $Z$ along $T$ at $t$ " was coined by him to describe that situation. The condition is on the ideal defining $Z$ locally at $t$ in some nonsingular ambient space. If we drop the requirement that $T$ be nonsingular, but instead assume that $T$ is the image of a section $\sigma$ of a morphism $Z \rightarrow T$ (as in our situation), then essentially the same idea works for the normal flatness of $\sigma$ (see (2.4)): the condition is now 
on the ideal of $Z$ viewed as embedded locally at $t$ in a neighborhood of the 0 section of $\mathbf{A}_{T}^{r}$ for suitable $r$ (we may assume $\sigma$ is the 0 -section). Unfortunately, (2.4) does not explicitly involve the ideal of the original singularity $x \in X$ $\hookrightarrow \mathbf{A}_{k}^{r}$ (i.e. the special fibre of $Z$ over $T$ ) so it is not by itself suited to our purpose. Without going into great detail here, suffice it to say that the missing ingredients come out of the flatness of $Z$ over $T$ (not used for (2.4)), albeit in a surprisingly nontrivial way. The basic fact is that the same conditions which insure that a "standard base" (2.2) of the original ideal of $X$ in $\mathbf{A}_{k}^{r}$ at $x$ is stable under the deformation, also insure that (2.4) is satisfied. Thus everything works out in the end: we finally get the conditions (2.13)-(2.14) for the normal flatness of a deformation which seem quite natural, and which provide the technical foundation for everything else. We note that one reason for the difficulty of this analysis is undoubtedly the fact that a standard base is highly unstable in general, and must be carefully nurtured through every infinitesimal stage if it is to survive (see (2.10), (2.12) and the subsequent examples).

Roughly speaking, we can distinguish two general directions along which the theory can now develop. The first is essentially a study of the smoothness properties of the functors $\Re_{j}$, or equivalently of the loci $N_{j}$. The "absolute" form of this question is that of obstructions in the $\Re_{j}$ themselves. The question, however, has a relative form suggested by Hironaka, which may admit a more comprehensive answer: Is the canonical morphism (denoted $\mathbf{u}$ ) from the normally flat deformations (i.e. the functor $\Re$ ) to the cone deformations which was mentioned above a smooth morphism? We first note that the answer to this question is affirmative, and moreover the functors $\boldsymbol{\pi}_{j}$ are unobstructed in the case when $X$ is a strict complete intersection at $x$, i.e. when the tangent cone to $X$ at $x$ is a complete intersection ((2.16)-(2.18)). These facts follow easily from the criteria (2.13) and (2.14). In the case of hypersurfaces, using the differential characterization of the $N_{j}$ (see especially (1.5.3)), the smoothness can be proved directly without substantial difficulty. For more general strict complete intersections, however, this approach appears to be much too complicated; indeed, going in the other direction, the smoothness of the $N_{j}$ should be expected to give a simplifying insight into the structure of certain differential loci-but we will not pursue this here.

In $\$ 3$ we work out one example $\left({ }^{2}\right)$ of a nonstrict complete intersection where everything turns out to be smooth: the normally flat deformations, the cone deformations, and the morphism u. However I see no reason to expect that the $\tau_{j}$ are smooth even for general complete intersections which are not strict, although I have no counterexample in hand. Moreover, the question of the smoothness of $\mathbf{u}$ is completely open (except for the strict complete intersections as noted above), but here there seems to be a chance of an affirmative

$(2) \S 4$ does not depend on $\$ 3$, so that $\$ 3$ may be omitted in a first reading. 
answer in general. In any case there is good evidence that a suitably formulated obstruction theory for $\mathbf{u}$, as well as for the functors $\boldsymbol{\vartheta}_{j}$ themselves, can shed light on a variety of geometric problems, and moreover will be seen to have considerable intrinsic interest. At the moment, for the purposes of this paper, we will content ourselves with the few indications already mentioned.

Now the second general direction, in contrast to the first, shows all its major features already in the case of hypersurfaces, i.e. it is not concerned with problems stemming from the ideal theoretic complexity of the singularity. On the contrary, the essential ideas are most visible in the strict complete intersection case, because of the smoothness of the $N_{j}$ in that case. We deal here with those aspects of the deformation theory which can be understood in terms of sections. It turns out that this point of view, if followed consistently to its natural conclusions, gives an approach to the general problem of stratification of moduli. The program which is suggested has yet to be carried out in full; the first stages are found in $\$ 4$ of this paper, and we now describe the main ideas:

We consider the versal deformation $\pi: \mathfrak{X} \rightarrow S$ of the singularity $X, \mathrm{x}$. The first consequence of the inclusion of sections in the functors $\varkappa_{j}$ was that the parameter spaces $N_{j}$ lie in $\mathfrak{X}$-the "total space" of the versal family-rather than in $S$, the moduli space of $X$ in the usual sense. The mapping $N_{j} \rightarrow S$ induced by $\pi$ represents the "forgetful" morphism of functors from $\boldsymbol{\vartheta}_{j}$ te the deformation functor of $X$; the morphism forgets the section. We will refer to such a section in $\Re_{j}$ as a " $j$-section". Let $S_{j}$ denote the image of $N_{j}$ in $S$. Then we have the following facts:

(i) A $j$-section (or indeed any section at all) of $\mathfrak{X}$ over $S_{j}$ may not exist; here is a situation where taking prorepresentable hulls of functors fails to commute with taking the image of a morphism of functors.

(ii) $N_{j}$ "acquires" singularities by $\pi$, i.e. $N_{j}$ is "less singular" than $S_{j}$. We can see this precisely in the strict complete intersection case in characteristic $0:\left({ }^{3}\right)$ then $N_{j}$ is smooth, and is the normalization of $S_{j}$; this follows from the smoothness together with the "completeness" of the modular family $\mathfrak{X} / \mathrm{S}$ at every "point" of $S$ [14].

Now there is a precise relationship between (i) and (ii): Look at the tangent spaces $t_{\Upsilon_{j}}$ of the functors $\Upsilon_{j}$, or, what amounts to the same thing, at the Zariski tangent spaces $T_{N_{j, x}}$ of $N_{j}$ at $x$, where $x$ is viewed as a point in the special fibre $X$ of $\mathfrak{X}$ over $S$. We prove in $\$ 4$ that we have a vector space decomposition $T_{N_{j}}=K_{j} \oplus T_{j}^{1}$ where $K_{j}$ is contained in the tangent space to the fibre of $\mathfrak{X} / S$ at $x$, i.e. in $T_{X, x}$, and $T_{j}^{1}$ is contained in $T_{S, s}$ where $s$ is the special point. Moreover, $K_{j}$ can be interpreted as the space of $j$-sections of the trivial 1st order deformation of $X$, and $T_{j}^{1}$ is the common part of the tangent

(3)In char $p N_{j}$ is a purely inseparable cover of the normalization [14]. 
spaces of $N_{j}$ and $S_{j}$. Thus (say, when $N_{j}$ is smooth), $S_{j}$ is singular "precisely" to the extent to which $X$ admits nontrivial $j$-sections of the trivial 1 st order family! $W$ must resolve these singularities before we can actually realize the sections. The reader will note the analogy here with the situation that arises in connection with the "level surface" deformation $f=t$ of, say, a hypersurface $f=0$. Then, as has been remarked for example in the context of monodromy questions, we must pass to a ramified cover of $k[[t]]$ in order to get a section. Actually, our remarks above apply only to $j>0$, for $N_{0}$ is all of $\mathfrak{X}$.

We note that $T_{j}^{1}$ may be defined as the $j$ th piece of $T_{X}^{1}$ (the tangent space to $S$ at $s$, or equivalently to the deformation theory of $X$ ) with respect to a certain filtration induced by the $\mathrm{m}$-adic one $(\mathrm{m}=$ the maximal ideal of $x$ in $X)$ with regard to a standard base for the ideal $J$ embedding $X$ locally at $x$ in a nonsingular ambient space (4.4); this all makes sense, because $T_{X}^{1}$ is a quoteint of $\operatorname{Hom}_{\theta}\left(J / J^{2}, \theta\right)$, where $\theta=\theta_{X, x}$. The filtration in question is the dual of the one that figures in our analysis in $\S 2$. As for $K_{j}$, in the sense of a subspace of $T_{X, x}$ it may also be characterized as the tangent space to the subscheme of $T_{X, x}$ which leaves the $j$ th infinitesimal neighborhood of the vertex in $C_{X, x}$ invariant by translation; recall that $C_{X, x}$ is the tangent cone to $X$ at $x$, with $C_{X, x} \hookrightarrow T_{X, x}$. In particular, for $j$ sufficiently large so that $\boldsymbol{\vartheta}_{j}=\Re, K_{j}$ is the "strict tangent space" $T_{X, x}$ : the largest linear subspace which splits off $C_{X, x}$. In conclusion, I want to give one indication of how this set-up can be expected to give some insight into stratification. We restrict ourselves now to the hypersurface case for simplicity; in practice, any results here should go over at least to the general strict complete intersections without substantial difficulty. We want to think of $S_{j}$ as a "higher discriminant locus" of $\pi: \mathfrak{X} \rightarrow S$; it consists of those points of $S$ whose fibre in $\mathfrak{X}$ contains at least one point of multiplicity $\geqslant j$. We have observed that the singularities of $S_{j}$, very nonisolated in general, are resolved by normalization $N_{j}$. The singularity at the special point is measured up to first order by $K_{j}$, since that is the tangent space to the special fibre of $S_{j}$ in $N_{j}$. Moreover, we can get our hands on the common tangent directions $T_{j}^{1}$ to both $S_{j}$ and $N_{j}$. Now we can define a subvariety of $S_{j}$, denoted $B_{j}$, which we call the " $j$ th transversal discriminantal singularity". Intuitively, $B_{j}$ contributes the singularity to $S_{j}$, for its fibre in $N_{j}$ is the same as that of $S_{j}$. The idea is to view $S_{j}$ itself as a family parametrized by another nonsingular subspace of itself which is tangent to $T_{j}^{1}$, and which we will also denote $T_{j}^{1}$ for simplicity. The special fibre of this family is $B_{j}$ :

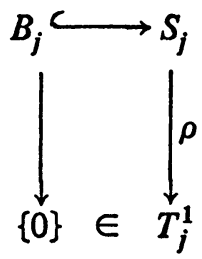


Now we want to consider the equisingular stratification of $B_{j}$ : Since $B_{j}$, like $S_{j}$, should be resolved by normalization, there is no trouble making sense of such a stratification; in our situation we call the strata "transversal strata". We stress that we are talking here about an intrinsic stratification of $B_{j}$, which a priori is unrelated to any stratification according to the fibres in $\mathfrak{X}$. However, let us denote by $\mathfrak{X}_{j}$ a neighborhood of $N_{j}$ in $\pi^{-1}\left(S_{j}\right) \subset \mathfrak{X}$, which does not contain any point of a fibre which is a singularity of multiplicity less than $j$ (i.e. for $j^{\prime} \leqslant j, \mathfrak{X}_{j} \cap N_{j^{\prime}}=N_{j}$ ). In our algebraic setting, we could take $\mathfrak{X}_{j}$ to be a formal neighborhood. Then, conjecturally, $\mathfrak{x}_{j}$ is locally trivial over each transversal stratum. Note that one meaning of this conjecture is that the "lattice of equisingular strata" of $B_{j}$ is the same as the lattice of jumpphenomena for singularities of multiplicity at least $j$ near $X$, and that $\mathfrak{X}_{j} \times_{S} B_{j} \stackrel{\mathrm{pr}_{2}}{\longrightarrow} B_{j}$ is the versal jump deformation for multiplicity at least $j$.

We should remark here that recent work of Giraud [15], [16] shows that the normally flat locus $N \hookrightarrow \mathfrak{X}$ has maximal contact with $X$ (viewed as the special fibre of $\mathfrak{X}$ of $S$ ) in that $N$ contains the infinitely near Hilbert-Samuel stratum of $X$ in a suitable sense (loc. cit.). In this regard one may ask for a deformationtheoretic interpretation of the question of obtaining a sufficiently "effective" maximal contact space, i.e. effective for general desingularization problems.

It is my great pleasure to acknowledge the support I received during various stages of the preparation of this work, above all in the form of interest, encouragement and concrete suggestions, on the part of H. Hironaka and $M$. Schlessinger; I thank them heartily. My deep thanks also go to Isolde Field, who typed the manuscript, and provided invaluable assistance in its preparation.

1. The versal normally flat families.

(1.0) Preliminaries on sections which are n-flat. Suppose we are given a cartesian diagram of schemes

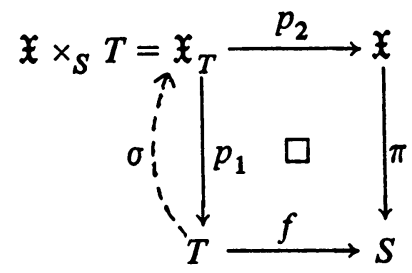

where $\sigma: T \rightarrow \mathfrak{X}_{T}$ is a section of $p_{1}$, i.e. $p_{1} \sigma=1_{T}$. Let $I_{\sigma}$ (or just $I$ where there is no danger of confusion) denote the ideal sheaf of $\hat{\Theta}_{\mathfrak{X}_{T}}$ defining $\sigma$. We denote by $T_{\sigma}^{n}$ (or just $T^{n}$ ) the $n$th infinitesimal neighborhood of $\sigma$ in $\mathfrak{X}_{T}$, i.e. $\theta_{\mathfrak{X}_{T^{n}}}=\theta_{\mathfrak{X}_{T}} / I^{n+1}$. We then have embeddings $i_{n}: T^{n} \hookrightarrow \mathfrak{X}_{T}, \sigma_{n}: T \hookrightarrow T_{n}$, with $\sigma$ $=i_{n} \sigma_{n}$ : 


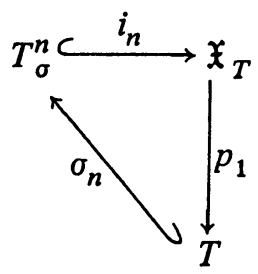

Now given $\mathfrak{X} / \mathrm{S}$ and an arbitrary morphism $f: T \rightarrow S$, our immediate goal is: For each integer $n \geqslant 0$, to find conditions on $f$ such that there exists a section $\sigma$ as above for which $\theta_{T^{n}}$ is flat over $\theta_{T}$. To do this, we use the "sheaf of principal parts of order $n$ of $\mathfrak{X}$ over $S$ ", denoted $P_{\mathfrak{X} / S}^{n}$. With the notations of the preceding paragraph, this is just $T^{n}$ in the special case where $T=\mathfrak{X}, f=\pi$, and $\sigma$ is the diagonal $\left({ }^{4}\right)$. We assume that the morphism $\pi$ is of finite type. Then $P_{\mathfrak{X} / S}^{n}$ is a coherent sheaf of $\theta_{\mathfrak{X}}$-algebras. Now we take the flattening stratification of $\mathfrak{X}$ corresponding to $P_{\mathfrak{X} / S}^{n}$. This is by definition a family $\left\{\mathfrak{X}_{n e}\right\}_{e \in Z_{0}}$ of locally closed subschemes of $\mathfrak{X}$ whose underlying point sets are pairwise disjoint and cover $\mathfrak{X}$, and which has the property: Given any morphism $g: T \rightarrow \mathfrak{X}, g^{*}\left(P_{\mathfrak{X} / S}^{n}\right)$ is a nat $\theta_{T}$-module if and only if $g$ factors through the natural map $\amalg_{e} \mathfrak{X}_{n e} \rightarrow \mathfrak{X}$ induced by the inclusions $j_{e}: \mathfrak{X}_{n e} \hookrightarrow \mathfrak{X}$. In fact the underlying point set of $\mathfrak{X}_{n e}$ is

$$
\left\{x \in \mathfrak{X} / \operatorname{dim}_{k(x)}\left(P_{\mathfrak{X} / S}^{n} \otimes_{\mathcal{O}_{\mathfrak{X}}} k(x)\right)=e\right\},
$$

and $g$ factors through $j_{e}$ if and only if $g^{*}\left(P_{\mathfrak{X} / S}^{n}\right)$ is locally free of rank $e$. The existence of flattening stratifications is proved in [2, Lecture 8].

Now suppose we have a diagram (1.0.1) with section $\sigma$ as above. Let $g=p_{2} \sigma$. Then with notations as above, we have

(1.0.2) LeMma. $g^{*}\left(P_{X / S}^{n}\right)=\hat{\theta}_{T^{n}}$.

Proof. $g^{*}\left(P_{\mathfrak{X} / S}^{n}\right)=\sigma^{*}\left(p_{2}^{*} P_{\mathfrak{X} / S}^{n}\right)=\sigma^{*}\left(P_{\mathfrak{X}_{T / T}}^{n}\right)$ since the formation of the sheaf of principal parts commutes with base extension $[1,16.4 .5]$. Now we use the following fact $[1,16.4 .11]$ : If $W \rightarrow U$ is a morphism with section $\sigma$, then $\boldsymbol{\sigma}^{*}\left(P_{W / U}^{n}\right)$ is isomorphic to $\theta_{U^{n}}$ viewed as $\theta_{U}$ algebra, where as usual $U^{n}$ denotes the subscheme of $W$ defined by the $n+1$ st power of the ideal of $\sigma$, with the structure of $U$-scheme on $U^{n}$ given by composing the inclusion $U^{n} \hookrightarrow W$ with the structure map $W \rightarrow U$. Thus in our case $\sigma^{*}\left(P_{\mathfrak{X}_{T / T}}^{n}\right)$ is $\theta_{T^{n}}$. Q.E.D.

Hence $\theta_{T^{n}}$ is locally free over $\theta_{T}$ of rank $e$ if and only if $g$ factors through $j_{e}: \mathfrak{X}_{n e} \hookrightarrow \mathfrak{X}$. Note that $\sigma$ is uniquely determined by $g$, in fact $\sigma=\left(1_{T}, g\right): T$ $\rightarrow T \times_{S} \mathfrak{X}=\mathfrak{X}_{T}$.

Conversely, suppose $f$ factors through $j_{e}$, i.e. $f=\pi j_{e} f^{\prime}$ for some $S$-morphism $f^{\prime}: T \rightarrow \mathfrak{X}_{n e}$.

( ${ }^{4}$ )The reader is referred to $[1,16.3 \mathrm{ff}]$, for details on these $P_{\mathfrak{z} / S}^{n}$. 


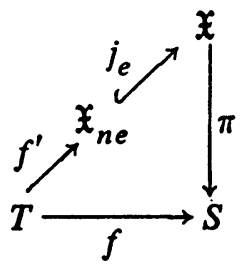

Let $g=j_{e} f^{\prime}$. Then $\left(1_{T}, g\right)$ defines an $S$-morphism $\sigma: T \rightarrow \mathfrak{X}_{T}$, and since $p_{1} \sigma=1_{T}, \sigma$ is a section. Then as above, since $g$ factors through $j_{e}, g^{*}\left(P_{X / S}^{n}\right)$ $=\theta_{T_{0}^{n}}$ is free of rank $e$.

The functoriality of the situation is seen as follows: Let $f: T \rightarrow S$ and suppose $\sigma$ is a section of $p_{1}: \mathfrak{X}_{T} \rightarrow T$ with $\hat{\theta}_{T_{a^{n}}}$ flat of rank $e$. Let $h: T^{\prime} \rightarrow T$ be a morphism. $\sigma$ induces a section $\sigma^{\prime}$ of $p_{1}^{\prime}: \mathfrak{X}_{T^{\prime}} \rightarrow T^{\prime}$, namely $\sigma^{\prime}=\left(1_{T^{\prime}}, p_{2} \sigma h\right)$.

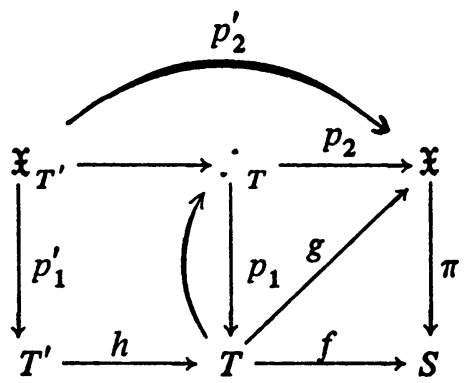

It is then easy to see that the flatness of $\theta_{T_{0}^{n}}$ implies that of $\theta_{T_{0}^{\prime \prime}}$ of the same rank. In fact, if we let $g=p_{2} \sigma$ and $g^{\prime}=p_{2}^{\prime} \sigma^{\prime}$, then as we have seen above the flatness of $\theta_{T_{o}^{n}}$ (resp. $\theta_{T_{o}^{\prime \prime}}$ ) is equivalent to the factorization of $g$ (resp. $g^{\prime}$ ) through $j_{e}$. But since $g^{\prime}=g h$ if $g$ factors through $j_{e}$ so does $g^{\prime}$.

We summarize:

(1.0.3) Proposition. Let $\pi: \mathfrak{X} \rightarrow S$ be a morphism of finite type, and let $n$ and $e$ be nonnegative integers. Let $F_{\pi n e}$ denote the contravariant functor from $S$ schemes to sets defined by

$$
F_{\pi n e}(T)=\left\{\sigma \mid \sigma \text { is a section of } p_{1}: \mathfrak{X}_{T} \rightarrow T \text { with } \theta_{T_{0}^{n}} \text { flat over } \theta_{T} \text { of rank } e\right\} .
$$

Then $F_{n n e}$ is represented by $\mathfrak{X}_{n e}$ (the rank e stratum in the flattening stratification of $P_{\mathfrak{X} / S}^{n}$ with inclusion $\left.j_{e}: \mathfrak{X}_{n e} \rightarrow \mathfrak{X}\right)$ viewed as $S$-scheme via the composition $\pi j_{e}$; the universal section is $\left(\mathfrak{X}_{\mathfrak{X}_{n e}}, j_{e}\right): \mathfrak{X}_{n e} \rightarrow \mathfrak{X}_{n e} \times_{S} \mathfrak{X}$.

(1.0.4) REMARK. Since any morphism $T \stackrel{\&}{\rightarrow} \mathfrak{X}_{n e}$ gives $T$ an $S$-scheme structure (after composition with $\pi$ ), we may view $F_{\pi n e}$ as defined on the category of all schemes, with

$$
F_{\pi n e}(T)=\left\{(f, \sigma) \mid f: T \rightarrow S \text { and } \sigma \text { a section of } X_{T}\right. \text { as above }
$$

where now $X_{T}$ denotes pullback over this $\left.f\right\}$. 
In this case as well, $F_{\pi n e}$ is represented by $\mathfrak{X}_{n, e}$, as is easily checked. The situation is of course the same if we are viewing the whole structure over some base $k: \mathfrak{X}_{n, e}$ represents the functor of sections on the category of $k$-schemes. We will use the terminology: If $Z \rightarrow T$ is a morphism with section $\sigma$, we will say " $\sigma$ is $n$-flat" if $T_{\sigma}^{n}$ is flat over $T$.

(1.0.5) REMARK. Given $Z \rightarrow T$ with section $\sigma$ defined by an ideal $I \subset \theta_{Z}, \sigma$ is $n$-flat for all $n \leqslant N$ if and only if $\mathrm{Gr}_{I}^{n}\left(\theta_{Z}\right)$ is flat over $\theta_{T}=\theta_{Z} / I$ for $n \leqslant N$. This is easy to check by induction on $n$, using the exact sequences $0 \rightarrow I^{n} / I^{n+1}$ $\rightarrow O_{Z} / I^{n+1} \rightarrow O_{Z} / I^{n} \rightarrow 0$.

(1.1) Normally flat deformations of order $j$. Let $X$ be an algebraic $k$-scheme $(k$ a field). Assume that either $X$ is proper over $k$ or that $X$ is affine with only isolated singularities. We recall the definition of the versal formal deformation of $X$, whose existence is proved in [4]: There is a complete local $k$-algebra $R$ and a flat morphism $\pi: \mathfrak{X} \rightarrow S=\operatorname{Spec}(R)(5)$ with the properties:

(i) If $s_{0}$ denotes the closed point of $S$ the fibre $\mathfrak{X}_{s_{0}}$ is $X$.

(ii) If $T$ is a local artin $k$-scheme and $p: Z \rightarrow T$ is a flat $k$-morphism with closed fibre $X$, then there is a $k$-morphism $f: T \rightarrow S$ for which $T \times_{S} \mathfrak{X}=\mathfrak{X}_{T}$ $\approx Z$ as $T$-scheme, i.e. there is a commutative diagram

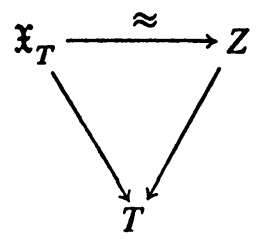

This may be expressed functorially as follows: Let $D_{X}$ (or $D$ when there is no danger of confusion) be the contravariant functor from local artin $k$-schemes to sets defined by

(a) $D(T)=$ the set of $T$-isomorphism classes of flat $k$-morphisms $p: Z \rightarrow T$ with closed fibre $X$.

(b) $T^{\prime} \rightarrow T$ induces $D(T) \rightarrow D\left(T^{\prime}\right)$ by $Z \mapsto Z_{T^{\prime}}=T^{\prime} \times_{T} Z$. Then with the terminology of [4], $S$ is a "prorepresentable hull" of $D$. ( $S$ fails to prorepresent $D$ in the strict sense because the morphism $f$ of (ii) above is in general not unique.)

(1.1.1) Now let $x$ be a $k$-rational point of $X$. For each integer $j \geqslant 0$, we define a contravariant functor $\Re_{j, X, x}$ (or just $\varkappa_{j}$ when there is no danger of confusion) from artin local $k$-scheme to sets by:

(a) $\vartheta_{j}(T)$ is the set of all pairs $(Z, \sigma)$ where $Z \in D(T)$ and $\sigma$ is a section of $Z \rightarrow T$ which meets the closed fibre in $x$ and which is $n$-flat for all $n \leqslant j$.

$(5)$ Strictly speaking, this formulation is valid only in the affine case. In the proper case, all we can assume is that $X$ is a formal scheme and $S=\operatorname{Spf}(R)$. 
(b) $T^{\prime} \stackrel{h}{\rightarrow} T$ induces $\Re_{j}(T) \rightarrow \Re_{j}\left(T^{\prime}\right)$ by assigning to $\sigma$ the section $\sigma^{\prime}$ $=\left(1_{T^{\prime}}, \sigma \circ h\right)$ of $p_{1}: Z_{T^{\prime}}=T^{\prime} \times_{T} Z \rightarrow T^{\prime}$.

Note that in virtue of the remark (1.0.4) and (a) above, a pair $(Z, \sigma)$ is in $\varkappa_{j}(T)$ if and only if the normal cone to $\sigma$ in $Z$ is flat over $T$ through degree $j$.

(1.1.2) Terminology. A pair $(Z, \sigma)$ as in (a) above is called a "normally flat deformation of order $\mathrm{j}$ of $X$ at $x$ "; we say that $\sigma$ is a "normally flat section of order $j$ " or a " $j$-section", or even that " $Z$ is normally flat along $\sigma$ of order $j$ ". (This recalls the terminology of Hironaka who defined the normal flatness of $Z$ along $Y$ to be the flatness (over $Y$ ) of the normal tone to $Y$ in $Z$.) If $T$ is $j$-flat for all $j$, it is simply called a "normally flat section", and the pair $(Z, \sigma)$ is then a "normally flat deformation". The functor of normally flat deformations is denoted $\varkappa_{X, x}$ or just $\varkappa_{\text {. We will see below that }} \pi=\varkappa_{j}$ for $j$ sufficiently large.

(1.1.3) REMARK. Each functor $\Re_{j}$ admits a morphism $a_{j}$ to the basic deformation functor $D$ of $X$; this morphism sends the pair $(Z, \sigma)$ to just $Z$, i.e. it forgets the section. We will see shortly in (1.2) that the functors $\varkappa_{j, X, x}$ admit prorepresentable hulls $N_{j}$ which embed naturally in the total space $\mathfrak{X}$ of the versal deformation $\mathfrak{X} / S$ of $X$ :

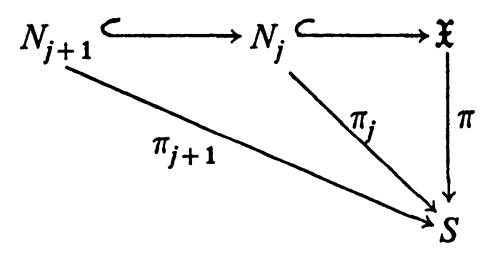

It is then not hard to see that the maps $\pi_{j}: N_{j} \rightarrow S$ induced by $\pi$ represent the morphisms of functors $a_{j}$. One of our goals will be to make a deeper study of $\pi_{j}$, and to interpret the singularities of its image. Considerable progress can be made in this direction when $X$ is a "strict complete intersection" (cf. (2.15)), for here the locus $N_{j}$ is smooth (2.18), and moreover-in characteristic 0 -is the normalization of its image in $S$ by $\pi_{j}$ [14].

(1.1.4) EXAMPLE. The family of plane curve singularities

$$
y^{3}+x^{4}-t x^{3}=0
$$

over $k[[t]]$ is normally flat along the 0 -section $x=y=0$; in fact the normal cone to this section is given by the ideal generated by $y^{3}+t x^{3}$ in $k[[t]][x, y]$, and is therefore flat over $k[[t]]$. By contrast, the family

$$
y^{3}+x^{4}-t x^{2}=0
$$

is only normally flat along $x=y=0$ of order 1 , since the cone is now given by $t x^{2}$, and is therefore not flat over $k[[t]]$ in degree 2 . We note however that the family 


$$
y^{3}+x^{4}-t^{2} x^{2}=0
$$

viewed now over $k[[t]] / t^{3}$, is normally flat along the section $y=0, x$ $=(1 / \sqrt{6}) t$. In fact, if we let $z=x-(1 / \sqrt{6}) t$, then in terms of the coordinates $y, z$ the family is $y^{3}+z^{4}+(4 / \sqrt{6}) t z^{3}$, and the section is $y=z=0$. Thus the cone is given by $y^{3}+4 / \sqrt{6} t z^{3}$, which is of course flat over $k[[t]] / t^{3}$. We remark that the phenomenon of this last example (3), where we have a family which is trivial up to some finite order, but which admits a nontrivial $j$-section, is essentially the same as the phenomenon of singularities in the image of the $\pi_{j}$ (see the remark (1.1.2) above); these ideas will be exposed later, beginning in $\$ 4$.

(1.2) The versal family. We now construct the versal normally flat family of order $j$ for $X$ at $x$. The basic components of the construction are the result (1.0.3) on the representation of the functor of $n$-flat sections of a given morphism, and the "ordinary" versal deformation $\mathfrak{X} / S$ of $X$. The upshot is that the functor $\vartheta_{j}$ has a prorepresentable hull $N_{j}$ which is naturally embedded in $\mathfrak{X}$, and which may be thought of intuitively as follows: $N_{j}$ is the "locus of points" in $\mathfrak{X}$ which, when viewed in their fibre over $S$, have a Hilbert-Samuel function (see below) which agrees with that of $X$ at $x$ at the first $j$ integers. Of course, this kind of formulation is not useful in the framework of infinitesimal geometry which is convenient to deformation theory. This is why we rely on the flatness of the normal cone to a section-rather than the constancy of the Hilbert-Samuel function along the section-for our definitions. However in a classical geometric context the two concepts are equivalent. We now make things precise:

(1.2.1) Definition. The Hilbert-Samuel function $H_{X, x}^{(1)}$ of $X$ at $x$ is defined as follows: If $\theta=\mathcal{O}_{X, x}$ and $\mathbf{m}=\mathbf{m}_{X, x}$, then

$$
H_{X, x}^{(1)}(n)=_{\text {def }} \text { length }_{\mathcal{O}}\left(\mathcal{O} / \mathbf{m}^{n+1}\right) .
$$

For simplicity we will denote $H_{X, x}^{(1)}=H$. Then for every $n$ we have the subschemes $\mathfrak{X}_{n, H(n)}$ as in (1.0). Now since $P_{\mathfrak{X} / S}^{n}(x)=\mathcal{O} / \mathrm{m}^{n+1}$ (this is the special case $T=\{t\}$ of Lemma (1.0.2)), we see that $x$ is in $\mathfrak{X}_{n, H(n)}$ for all $n$. It follows from (1.0.4) that $\mathfrak{X}_{n, H(n)}$ represents the functor of sections of $\mathfrak{X} / S$ which are $n$ flat and pass through $x$ in the closed fibre. Now since $\mathfrak{X}_{n, H(n)}$ is locally closed in $\mathfrak{X}$ for all $n$, the subscheme

$$
N_{j}={ }_{\text {def }} \bigcap_{n=0}^{j} \mathfrak{X}_{n, H(n)} \cap \operatorname{Spec}\left(\Theta_{\mathfrak{X}, x}\right)
$$

is a nonempty closed subscheme of $\operatorname{Spec}\left(\theta_{\mathfrak{X}, x}\right)$ for all $j$, and we get:

(1.2.3) $N_{j}$ represents the functor of sections of $\mathfrak{X} / S$ which are $n$-flat for all $n \leqslant j$-i.e. normally flat of order $j$-and which pass through $x$ in the closed 
fibre, if we restrict this functor to the category of local $k$-schemes. The universal section is $\left(1_{N_{j}}, i_{j}\right): N_{j} \rightarrow N_{j} \times_{S} \mathfrak{X}$, where $i_{j}$ is the inclusion. Note that $N_{j} \subset N_{j+1}$, so that the $N_{j}$ stabilize for some $j$ by the noetherian property. Thus

$$
N=\text { def } \bigcap_{j} N_{j}=\bigcap_{n} \mathfrak{X}_{n, H(n)} \cap \operatorname{Spec}\left(\Theta_{\mathfrak{X}, x}\right)
$$

is actually a finite intersection, and thus it is easy to see that: Over the category of local $k$-schemes $N$ represents the functor of normally flat sections of $\mathfrak{X} / S$ which pass through $x$; the universal section is $\left(1_{N}, i\right): N \times_{S} \mathfrak{X}$.

(1.2.4) THEOREM. $N_{j}$ is a hull of $\Re_{j}\left({ }^{6}\right)$; the versal normally flat deformation of order $j$ is

$$
N_{j} \times{ }_{S} \mathfrak{X} \stackrel{\mathrm{pr}_{1}}{\longrightarrow} N_{j}
$$

and the versal $j$-section is $\left(1_{N_{j}}, i_{j}\right): N_{j} \rightarrow N_{j} \times_{S} \mathfrak{X}$, where $i_{j}$ is the inclusion. $N=\bigcap_{j} N_{j}$ is a hull of the functor $\Re$ in the same sense; the intersection is actually finite.

Proof. We just put the components together: We know that $S$ is a prorepresentable hull of the ordinary deformation functor $D$ of $X$, and $X / S$ is the versal formal deformation. This means that if $h_{S}$ denotes the restriction to the category $\mathrm{C}=(($ artin local $k$-schemes $))$ of the functor of points of $S$, then the morphism $h_{S} \stackrel{f}{\rightarrow} D$ (given by pulling back $\mathfrak{X}$ ) is smooth, and is an isomorphism on tangent spaces. It follows immediately from the defintion of $\vartheta_{j}((1.1 .1)(a))$ that if $F$ denotes the restriction to $\mathcal{C}$ of the functor of $j$-sections of $\mathfrak{X} / S$ through $x$, then $f$ induces a morphism $F \rightarrow \Re_{j}$ which is also smooth and identifies tangent spaces. Finally, if $h_{N_{j}}$ is viewed as defined on $C_{\text {, (1.2.3) }}$ implies that $h_{N_{j}}$ and $F$ are isomorphic; and it is clear that the resulting smooth morphism $h_{N_{j}}=F \rightarrow \Re_{j}$ identifies the universal $j$-section of $\mathfrak{X} / S$ with a versal normally flat deformation of order $j$ of $\mathfrak{X}$.

(1.2.5) $N_{j}$ (and hence $N$ ) is finite over $S$. In fact, since we are starting with an isolated singularity, $N_{j}$ is quasi-finite over $S$, hence finite since $S$ is the spectrum of a complete local ring.

We remark that in any characteristic, we can start with $\hat{X}=\operatorname{Spec}\left(\hat{\theta}_{x, x}\right)$ (rather than $\mathfrak{X}$ ) over $S$; this amounts to considering the corresponding versalfamily of formal or "algebroid" singularities. Then if we define $\hat{N}_{j}$ for $\hat{x}$ as we $\operatorname{did} N_{j}$ for $\mathfrak{X}$, the $\hat{N}_{j}$ are finite over $S$. In fact, $\hat{N}_{j}$ is simply the spectrum of the completion of the local ring of $N_{j}$ at $x$.

(1.3) Our basic idea up to now has been that the flat strata of the $P_{X / S}^{n}$ both

$\left.{ }^{6}\right)$ Here we mean that if $h_{N_{j}}$ denotes the restriction of the functor of points of $N_{j}$ to the category of artin local $k$-schemes, there is a smooth morphism $h_{N} \rightarrow x_{j}$ which is an isomorphism on tangent spaces. 
represent the functor of $n$-flat sections, and give the constant loci for the "relative Hilbert-Samuel function", i.e. the Hilbert-Samuel function of the fibres. Thus, intuitively it is equivalent to say that a section is normally flat of order $j$, and that the first $j$ values of the relative Hilbert-Samuel function remain constant along the section (this last condition, of course, has nontrivial content only when $\operatorname{dim}(S)>0$, whereas the first makes sense infinitesimally). Now this "equivalence" has been justified by the fact that at rational points of the fibres, i.e. at points $y \in \mathfrak{X}$ for which $\kappa(y)=\kappa(s)$ where $s=\pi(y)$ and $\kappa$ denotes residue field, we have a canonical identification:

$$
P_{\mathfrak{X} / S}^{n}(y)=\theta_{\mathfrak{X}_{s, y}} / \mathbf{m}_{\mathfrak{X}_{s, y}}^{n+1}
$$

It is natural to ask, however, what is the relationship between the $P^{n}$ and the Hilbert-Samuel function at arbitrary (nonrational) points of the fibres. In this regard, it is easy to see that (*) still holds when $\kappa(y)$ is separable (algebraic) over $\kappa(s)$ : The problem is just on the fibre, so we may assume $S=\operatorname{Spec}(\kappa(s))$. Then we reduce to the rational case after a base extension to get $\mathfrak{X}^{\prime}$ $=\mathfrak{X} \times_{\kappa(s)} \kappa(y)$; the point is that the base extension commutes with the $P^{n}$ 's, and is étale because of the separability, so the truncated local rings at $y$ in $\mathfrak{X}$ and at any $\kappa(y)$-rational point $y^{\prime}$ in $\mathfrak{X}$ lying over $y$ are identified. Note that the coherence of the $P_{\mathfrak{X} / S}^{n}$ (when $\mathfrak{X} / S$ is locally of finite type) together with (*), give a proof of the upper semicontinuity of the relative Hilbert-Samuel function when $S$ is of residue characteristic 0 at all points.

Now in the inseparable case of $\kappa(y) / \kappa(s),(*)$ is false-in fact any inseparable field extension $L / K$ gives a counterexample: $P_{L / K}^{n}$ is generated as $L$-algebra by the differentials of elements in a $p$-base for $L$ over $K$. Similarly, upper semicontinuity of the relative $\mathrm{H}-\mathrm{S}$ function fails quite decisively in the presence of inseparablity $\left({ }^{7}\right)$. As a first example, let $K$ be a field of characteristic $p$ with $a \in K-K^{p}$. Let

$$
S=\mathbf{A}_{K}^{1}=\operatorname{Spec} K[X], \quad \mathfrak{X}=\operatorname{Spec}\left(K[X, Y] /\left(Y^{p}-a\right)\right),
$$

and $\pi: \mathfrak{X} \rightarrow S$ the projection on the $X$-coordinate. Let $s \in S$. If $\kappa(s)$ contains $\sqrt[P]{a}$ the fibre $\mathfrak{X}_{s}$ is isomorphic to $\operatorname{Spec}\left(\kappa(s)[Z] / Z^{p}\right)$, where $Z=Y-\sqrt[P]{a}$; here the fibre is singular. However, if $\sqrt[P]{a} \notin \kappa(s)$, then $\mathfrak{X}_{s}$ is the spectrum of the field $\kappa(s)(\sqrt[P]{a})$. Since there are infinitely many $s \in S$ with $\sqrt[P]{a} \in \kappa(s)$, the locus of points in $\mathfrak{X}$ where the relative $\mathrm{H}-\mathrm{S}$ function is larger than the constant function 1 is not a closed set.

Note that in this example, the uppersemicontinuity fails "globally", but "local" uppersemicontinuity, i.e. nondecreasing under specialization, is still valid

(7) This is in contrast with the behavior of the absolute H-S function on scheme $X$, which is always upper-semicontinuous whenever $X$ is excellent. cf. [6]. 
(the generic fibre of $\mathfrak{X} / S$ is a field). We now give an example, however, to show that local uppersemicontinuity can also fail: Again, let $a \in K-K^{p}$, and consider the irreducible plane curve

$$
C=\operatorname{Spec} K[X, Y] /\left(Y^{p}-a X^{p}\right) .
$$

Let $P_{1}$ be the origin, and let $P_{2}=(1, \sqrt[P]{a})$, i.e. the point defined by the ideal $\left(x-1, y^{p}-a\right) . P_{1}$ is a $K$-rational point at which $C$ is singular. The residue field at $P_{2}$ is $K(\sqrt[P]{a})$, and $C$ is nonsingular at $P_{2}$ (although $C$ fails to be "smooth" anywhere). Let $\theta_{i}$ denote the local ring of $C$ at $P_{i}, i=1,2$. We get our example by letting $\mathfrak{X}=\operatorname{Spec}\left(\theta_{1} \otimes_{K} \theta_{2}\right), S=\operatorname{Spec}\left(\theta_{1}\right)$, and $\pi=\mathrm{pr}_{1}$. In fact, since the closed point of $S$ is $K$-rational, the closed fibre looks like $\operatorname{Spec}\left(\theta_{2}\right)$ which is a regular scheme of dimension 1; its Hilbert-Samuel function is therefore $H(n)=n+1$ for all $n$. On the other hand, the generic point of $S$ is just the function field $F$ of $C$, which contains $\sqrt[p]{a}$. Thus the generic fibre of $\mathfrak{X}$ over $S$ is the local ring at $(1, \sqrt[p]{a})$ of the $p$-fold line $(Y-\sqrt[p]{a})^{p}=0$ in $\mathbf{A}_{F}^{2}$. Since this is not reduced, it is singular, and its H-S function is therefore larger than that of the closed fibre.

There are many intriguing aspects of this situation in characteristic $p$ which would take us too far afield to treat here in detail. We mention, however, one striking fact: It can be shown that the uppersemicontinuity of the relative $\mathrm{H}$ $S$ function for a morphism $\mathfrak{X} \rightarrow S$ when $S$ is local can fail only if $S$ is singular! This can be regarded as evidence of the "universality" of the above example in a suitable sense. A discussion of these matters will be published elsewhere. We finally note that Lejeune-Jalabert and Teissier [7] prove uppersemicontinuity and in fact the existence of stratifications for a certain modified relative H-S function which takes the inseparability into account (i.e. it behaves like the rank of the $P^{n}$ 's), and which agrees with the usual one in characteristic 0 .

(1.4) Suppose we have a family $Z / T$ with a section $\sigma$. Let $I$ denote the ideal of $\sigma$ in $\theta_{Z}$. We have remarked earlier (1.0.5) that $\sigma$ is a $j$-section if and only if $\operatorname{Gr}_{I}^{n}\left(\theta_{Z}\right)$ is flat over $\theta_{T}=\theta_{Z} / I$ for $n \leqslant j$. If moreover $Z$ is flat over $T$, it is easy to see that the flatness of these $\mathrm{Gr}^{n}$ 's is in turn equivalent to $I^{n}$ being flat over $\theta_{T}$ for $n \leqslant j+1$. Thus, in case $Z$ is flat over $T$, associated to a $j$-section $\sigma$ are the two flat graded $\theta_{T}$-algebras $\oplus_{n \leqslant j} I^{n} / I^{n+1}$ and $\bigoplus_{n<j+1} I^{n}$, as well as the sequence of flat $\theta_{T}$-algebras $\left\{\theta_{Z} / I^{n}\right\}_{n \leqslant j+1}$. Conversely, the flatness of any one of these three implies that of the other two, i.e., that $\sigma$ is a $j$-section. Of course, if $\sigma$ is normally flat, i.e. if it is a $j$-section for all $j$, then the full algebras $\operatorname{Gr}_{I}\left(\theta_{Z}\right)=\oplus_{n} I^{n} / I^{n+1}$ and $\operatorname{Pow}_{I}\left(\theta_{Z}\right)=\oplus_{n} I^{n}$ are flat over $\theta_{T}$.

(1.4.1) LEMMA. Let $B$ be a flat A-algebra, $I$ an ideal of $B$. Suppose $\mathrm{Gr}_{I}(B)$ is flat over $A$ through degree $j$ (equivalently, $\operatorname{Pow}_{I}(B)$ is flat through degree $j+1$ ). Let $A^{\prime}$ be an arbitrary $A$-algebra, $B^{\prime}=B \otimes_{A} A^{\prime}, I^{\prime}=I B^{\prime}$. Then

(i) $\mathrm{Gr}_{I^{\prime}}\left(B^{\prime}\right)=\mathrm{Gr}_{I}(B) \otimes_{A} A^{\prime}$ through degree $j$, 
(ii) $\operatorname{Pow}_{I^{\prime}}\left(B^{\prime}\right)=\operatorname{Pow}_{I}(B) \otimes_{A} A^{\prime}$ through degree $j+1$ (and consequently these algebras are flat over $A^{\prime}$ in the degrees indicated).

Proof. First note that we always have $B / I^{n} \otimes_{A} A^{\prime}=B^{\prime} / I^{\prime n}$ (with no hypothesis of flatness). Now as we have observed above, the flatness of $\mathrm{Gr}_{I}(B)$ through degree $j$ is equivalent to the flatness of the $B / I^{n}, n \leqslant j+1$. Thus

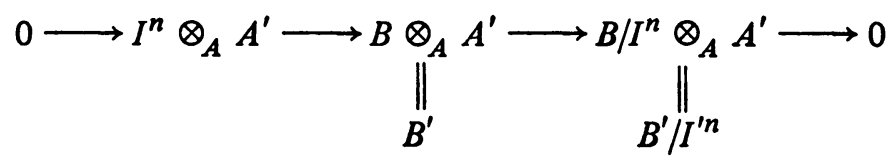

is exact for $n \leqslant j+1$, since $\operatorname{Tor}_{A}^{1}\left(B / I^{n}, A^{\prime}\right)$ maps onto the kernel. Hence $I^{\prime n}=I^{n} \otimes_{A} A^{\prime}$ for $n \leqslant j+1$, which is (ii). For (i), observe that the sequence

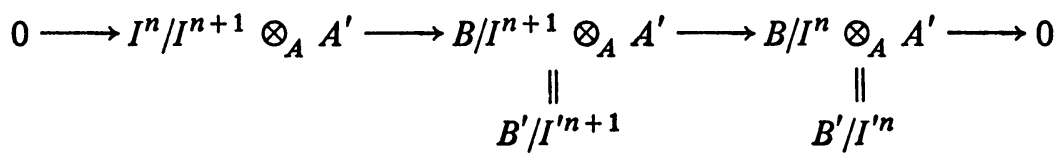

is exact for the same reason as above.

(1.4.2) Proposition. Suppose $(Z / T, \sigma)$ is a normally flat deformation of $X$ at $x$. Then

(i) $C_{\sigma / Z}$ (the normal cone to $\sigma$ in $Z$ ) is a flat cone deformation of $C_{X, x}$ (the tangent cone to $X$ at $x$ ) over $T$.

(ii) Let $X^{\prime}$ denote the blowing up of $x$ in $X$, and let $Z^{\prime}$ denote the blowing up of $\sigma$ in $Z$. Then $Z^{\prime}$ is a flat deformation of $X^{\prime}$ over $T$.

Proof. By definition, if $I$ is the ideal of $\sigma$ in $Z, C_{\sigma / Z}$ $=\operatorname{Spec}\left(\operatorname{Gr}_{I}\left(\theta_{Z}\right)\right)$ and $Z^{\prime}=\operatorname{Proj}\left(\operatorname{Pow}_{I}\left(\theta_{Z}\right)\right)$. Because $\sigma$ is a normally flat section, these are both flat over $T$, so the assertions (i) and (ii) correspond to (i) and (ii) of Lemma (1.4.1) (letting $A=\theta_{T}, A^{\prime}=A / N, N=$ the maximal ideal of the special point of $T$ ).

(1.4.3) Thanks to this proposition, we get morphisms of functors:

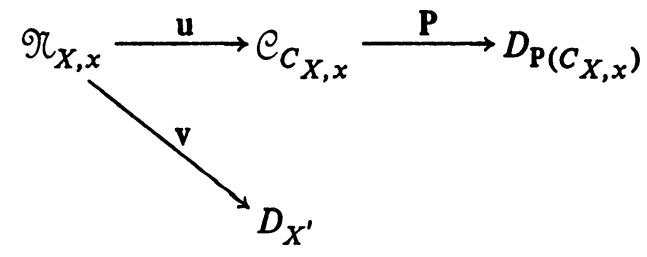

where $e_{C_{X, x}}$ denotes the functor of cone deformations of the tangent cone $C_{X, x}, D_{X^{\prime}}$ and $D_{\mathrm{P}\left(C_{X, x}\right)}$ denote the ordinary deformation functors of $X^{\prime}$ (the blowing up of $x$ in $X)$ and $\mathbf{P}\left(C_{X, x}\right)$ (the projectivized tangent cone); $\mathbf{u}$ (resp. v) associates to $(Z / T, \sigma)$ the normal cone to $\sigma$ in $Z$ (resp. the blowing up of $\sigma$ in 
$Z$ ), viewed again over $T$. Finally, $\mathbf{P}$ just replaces a family of cones by the corresponding family of projective varieties. Note that the image of $\mathbf{v}$ actually lands in the subfunctor of $D_{X^{\prime}}$ containing those deformations which carry along the exceptional fibre $F$ of $X^{\prime}$. Of course, $F=\mathbf{P}\left(C_{X, x}\right)$, and the induced deformation is given by the composition $\mathbf{P} \circ \mathbf{u}$.

(1.4.4) Some remarks on obstructions. One very basic question is whether the morphism $\mathbf{u}$ of (1.4.3) is smooth in general, i.e. for arbitrary singularities. This amounts to the following: Suppose we are given a normally flat deformation $(Z / T, \sigma)$, together with its associated cone deformation $u(Z, \sigma)=G$ over $T$. Suppose moreover that we have an embedding $T \hookrightarrow T^{\prime}$ and a cone deformation $G^{\prime} / T^{\prime}$ inducing $G / T$ (we suppose $T, T^{\prime}$ are artin local schemes). The smoothness means that for any such situation there exists a normally flat family $\left(Z^{\prime}, \sigma^{\prime}\right)$ over $T^{\prime}$, with $\mathbf{u}\left(Z^{\prime}, \sigma^{\prime}\right)=G^{\prime}$, and which induces $(Z, \sigma)$ over $T$ by restriction:

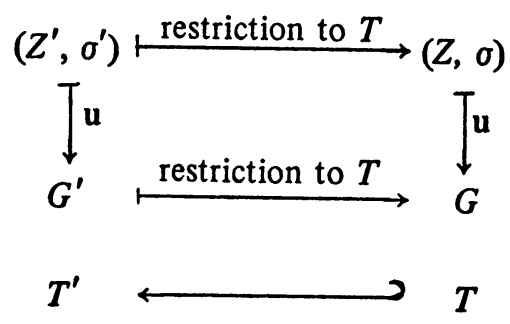

In $\S 2,(2.16)$ we show that this question has an affirmative answer when $X$ is a "strict complete intersection" at $x$. However at this moment the answer is unknown for any other class of singularities. We note that for the strict complete intersections, all the functors $\vartheta_{j}$ are smooth (2.17), which is a priori a separate question from the smoothness of $\mathbf{u}$ for general singularities.

In this paper we will not attempt to develop any systematic treatment of obstructions, although in $\$ 3$ we work out an example-not a strict complete intersection-where all obstructions vanish, including the "relative" obstruction to the smoothness of $\mathbf{u}$. We would also like to indicate here how-in contrast to the unsettled situation regarding $\mathbf{u}$-one can get examples of ordinary obstructions, i.e. cases where the functor $\vartheta_{X, x}$ (or equivalently the space $N_{X, x}$ ) is not smooth. We will require

a projective variety $V / k$, say nonsingular, with an obstructed deformation as an abstract variety; in other words a flat family $\checkmark / T$ ( $T$ an artin scheme) with closed fibre $V$, which does not extend to a flat family over some $T^{\prime} \hookleftarrow T$. Moreover, we suppose that this obstructed family $\mathscr{V} / T$ is projective, so that $\mathscr{\Upsilon} \hookrightarrow \mathbf{P}_{T}^{N}$, some $N$. 
For example, we can let $V$ be as in Mumford's paper [3]: He finds a curve $C \hookrightarrow \mathrm{P}^{3}$ with an obstructed embedded deformation theory (i.e. a singular Hilbert scheme) and blows it up in $\mathrm{P}^{3}$ to get $V$ (observing that the deformation theory of $V$ as abstract variety is the same as the embedded deformation theory of $C$ in $\mathbf{P}^{3}$ ).

In any case, given $\checkmark / T$ as in $(*)$, we may assume for the embedding $\checkmark \hookrightarrow P_{T}^{N}$ that for $\nu \geqslant 1$ all the higher cohomology of the sheaves $\theta_{\sqrt{ }}(\nu)$ vanishes (by composing $\checkmark \hookrightarrow \mathbf{P}^{N}$ if necessary with a suitably high " $n$-tuple" embedding $\left.\mathbf{P}^{N} \rightarrow \mathbf{P}^{N^{\prime}}\right)$. Then the family of cones

$$
G=\operatorname{Spec}\left(\bigoplus_{\nu=0}^{\infty} H^{0}\left(\theta_{\gamma}(\nu)\right)\right)
$$

is flat over $T$. The closed fibre of $G$ over $T$ is of course the cone over the embedding $V \hookrightarrow \mathrm{P}_{k}^{N}$. Let $X$ be this cone, $x$ the vertex (since $V$ is nonsingular, $x$ is an isolated singularity). Now $G$ is certainly a normally flat deformation of $X, x$-for any cone deformation is its own normal cone to the 0 -section. Moreover, if $G$ could be extended to a normally flat deformation $\left(Z^{\prime}, \sigma^{\prime}\right)$ over $T^{\prime}$, the normal cone to $\sigma^{\prime}$ gives a cone deformation $G^{\prime}$ which extends $G$. Hence we get an extension of ' $\checkmark$ to $T^{\prime}$ by taking the family of projective varieties over $T^{\prime}$ corresponding to $G^{\prime}$. This contradiction shows that $G / T$ must be obstructed as a normally flat deformation.

(1.5) Suppose $X$ is the hypersurface $f=0$ in $\mathbf{A}_{k}^{r}=\operatorname{Spec}\left(k\left[z_{1}, \ldots, z_{r}\right]\right)$, with only isolated singularities, one of which is at the origin $x$. In this case, as far as the constructions of this section are concerned, it is easy to see things very explicitly. As is well known, the versal deformation $\mathfrak{X} / S$ of $X$ may be described as follows: Let $g_{i}, i=1, \ldots, m$ be a set of representatives in $k\left[z_{1}, \ldots, z_{r}\right]$ of a $k$-basis of $k\left[z_{1}, \ldots, z_{r}\right] /\left(f, \partial f / \partial z_{1}, \ldots, \partial f / \partial z_{r}\right)$. Let $R$ be a formal powerseries ring over $k$ in variables $t_{1}, \ldots, t_{m}$. Then $S=\operatorname{Spec}(R)$, and $\mathfrak{X}$ is defined in $\mathbf{A}_{s}^{r}$ by the equation

$$
F(z, t)=f+\sum_{i=1}^{m} t_{i} g_{i}=0 .
$$

Now we want to compute $P_{\mathfrak{X} / S}^{n}$. For this, we first note

$$
P_{A_{S / S}^{\prime}}^{n}=\theta_{A_{S}^{\prime}}\left[d z_{1}, \ldots, d z_{r}\right] /\left(d z_{1}, \ldots, d z_{r}\right)^{n+1},
$$

a truncated polynomial algebra over $\theta_{A_{s}^{\prime}}$ in the symbols $d z_{j}$. In this algebra we denote by $d F$ the formal Taylor expansion through degree $n$ of $F$ with respect to the variables $z_{j}$ :

$$
d F==_{\operatorname{def}} \sum_{|\mu|=0}^{n} F_{\mu} / \mu ! d z^{\mu}
$$


(Here $\quad \mu=\left(\mu_{1}, \ldots, \mu_{r}\right),|\mu|=\mu_{1}+\cdots+\mu_{r}, \mu !=\mu_{1} ! \cdots \mu_{r} !, d z^{\mu}=d z_{1}^{\mu_{1}}$ $\cdots d z_{r}^{\mu_{r}}$, and $F_{\mu}=\partial^{|\mu|} F / \partial z_{1}^{\mu_{1}} \cdots \partial z_{r}^{\mu_{r}}$.) Then we may describe:

$$
P_{\mathfrak{X} / S}^{n}=P_{A_{S}^{\prime} / S}^{n} /(d F, F)=\mathcal{O}_{\mathfrak{X}}\left[d z_{1}, \ldots, d z_{r}\right] /(\overline{d F})+(d z)^{n+1}
$$

where $\overline{d F}$ is the image of $d F$ in $\mathcal{O}_{\mathfrak{X}}\left[d z_{1}, \ldots, d z_{r}\right]$; the reader will find these basic facts about the $P^{n}$ proved in $[1, \S 16]$.

Suppose we are given $y \in \mathrm{A}_{S}^{r}$ with $\pi(y)=s \in S$. If $\kappa(y)=\kappa(s)$, we see from the definition (1.5.3) that $d F$, evaluated at $y$, is just the Taylor expansion through degree $n$ around the point $y$ of the function induced by $F$ on the fibre $\mathbf{A}_{x(s)}^{r}$; in this expression the $d z$ 's play the role of the parameters. Combining this with (1.5.4), the isomorphism $P_{\mathfrak{X} / S}^{n}(y)=\mathcal{O}_{\mathfrak{X}_{s, y}} / \Re_{\mathfrak{X}_{s, y}}^{n+1}$ is evident. Now the Hilbert-Samuel function at a point of a hypersurface $f=0$ is completely determined by the multiplicity at that point, i.e. by the order of $f$ there. Thus in view of what we have observed above, the rank of $P_{\mathfrak{x} / s}^{n}$ at a point $y$ dependsin our case at hand-only on the smallest integer $\nu$ such that $F_{\mu}(y) \neq 0$ for some $\mu$ with $|\mu|=\nu$. Thus, as we expect:

(1.5.5) In the hypersurface case, with notation as above, $N_{j}$ is the component through $x$ of the subscheme of $\mathfrak{X}$ defined by the ideal generated by the $F_{\mu},|\mu| \leqslant j$.

(1.5.6) REMARK. For a general singularity $X, x$, the locus $N_{1}$ consists of those points of $\mathfrak{X}$ at which the Hilbert-Samuel function of the fibre, evaluated at 1 , is the same as that of $X$ at $x$. This means simply that the embedding dimension, i.e. the rank of $\pi / \pi^{2}$, remains constant. However, in the case of a hypersurface $X: f=0$ in $\mathbf{A}_{k}^{r}$, the embedding dimension at a point is $r$ if and only if $X$ is singular at the point (otherwise the dimension is $r-1$ ). Thus here $N_{1}$ may be described as the "critical locus" of the mapping $\mathfrak{X} \rightarrow S$, i.e. those points at which the fibre is singular. In fact, it is easy to see by induction that

(1.5.7) When $X$ is a hypersurface, and $j$ is $\leqslant$ the multiplicity of $X$ at $x, N_{j}$ is the "critical Jacobian locus" of the mapping $N_{j-1} \rightarrow S$; this is by definition the locus where the dimension of the kernel of the associated map on tangent spaces is greatest or, equivalently, where the dimension of the Zariski tangent space to the fibre is greatest $(8)$.

Whether (1.5.7) holds for arbitrary singularities seems to be an open question.

2. The equations defining normally flat deformations. In this section we use the following notation:

$\left.{ }^{8}\right)$ In the terminology of the Thom-Boardman theory of singularities of differentiable maps, (1.5.6) says that (in the hypersurface case): if $k_{1}, \ldots, k_{s}$ is the "symbol" of $\pi: X \rightarrow S$ at $x$, then for $j<\nu$ (the multiplicity of $X$ at $x$ ), $N_{j}$ is the Boardman variety $\Sigma^{k_{1}, \ldots, k_{j}}$ (cf. [11). 


$$
\left\{\begin{array}{l}
A \text { is a local ring, } \quad N=\max (A), \quad k=A / N, \\
B=A\left[\left[x_{1}, \ldots, x_{n}\right]\right], \quad M=\max (B), \\
J \subset(x) B \text { is an ideal of } B, \\
\mathcal{O}=B / J, \quad \mathbf{m}=\max (\mathcal{O}) .
\end{array}\right.
$$

Geometrically, this corresponds to a family of singularities over $\operatorname{Spec}(A)$, with section $x=0$. We want to find necessary and sufficient conditions for this section to be normally flat, i.e. so that $\mathrm{Gr}_{(x)}(\theta)$ is flat over $A$. This analysis has two stages. In the first-with no assumption of flatness on $\theta$ itself-we find that the flatness of the $\mathrm{Gr}$ is equivalent to the existence of an $M$-standard base $\left\{f_{i}\right\}_{i}$ for $J$ such that $\nu_{M}\left(f_{i}\right)=\nu_{(x)}\left(f_{i}\right)$ for each $i$ (Theorem (2.4); see below for definitions). The techniques here are analogous to those used by Hironaka [8] for a slightly different situation. From the perspective of deformation theory, however, the point is not to recognize a standard base of an ideal given abstractly over $A$. Thus, in the second stage, we assume that $\theta$ is a flat deformation over $A$ of $k\left[\left[x_{1}, \ldots, x_{n}\right]\right] / \bar{J}$, where we are given an $(x)$-standard base $\left\{\bar{f}_{i}\right\}_{i}$ of $J$. We show that if $J=\left\{f_{i}\right\}_{i} B$ with $f_{i}(\bmod N)=\bar{f}_{i}$ and $\nu_{(x)}\left(f_{i}\right)$ $=\nu_{(x)}\left(\bar{f}_{i}\right)$, then the $f_{i}$ are necessarily an $M$-standard base of $J((2.10))$; we can then invoke (2.4) to get the normal flatness. We then obtain the criterion (2.13), which is most germane to the study of the normally flat deformations of a given singularity.

For example, it follows easily from this that the normally flat deformations of a strict complete intersection (i.e. a singularity whose tangent cone is a complete intersection) are unobstructed, and that the morphism $u$ of (1.4.3) is smooth in this case ((2.16), (2.17)).

To begin our study, we first note that the inclusions $N^{\mu}(x)^{\nu} \subset M^{\mu+\nu}$ induce a map

$$
\beta: \mathrm{Gr}_{N}\left(\mathrm{Gr}_{(x)}(\theta)\right) \rightarrow \mathrm{Gr}_{\mathrm{m}}(\theta) .
$$

(2.1) $\beta$ is an isomorphism.

Proof. The surjectivity of $\beta$ follows from the commutativity of the diagram

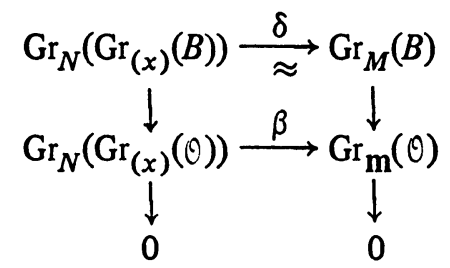

and the fact that $\delta$ is an isomorphism (both $\mathrm{Gr}_{N}\left(\mathrm{Gr}_{(x)}(B)\right)$ and $\mathrm{Gr}_{M}(B)$ are polynomial rings over $\mathrm{Gr}_{N}(A)$ in $\left.x_{1}, \ldots, x_{n}\right)$. Now suppose that $A$ is an artin 
local ring, and that $J$ contains some power of the ideal $(x)$. Then $\theta$ is an $A$ module of finite length, which is the same as the length of both $\operatorname{Gr}_{m}(\theta)$ and $\mathrm{Gr}_{N}\left(\mathrm{Gr}_{(x)}(\theta)\right)$. Thus $\beta$, being surjective, is also injective, so that $\beta$ is an isomorphism in this case.

Now, still assuming that $A$ is artin, let $\theta$ be arbitrary, and let $\theta_{p}$ denote $\theta /(x)^{p}$. Then we have a commutative diagram

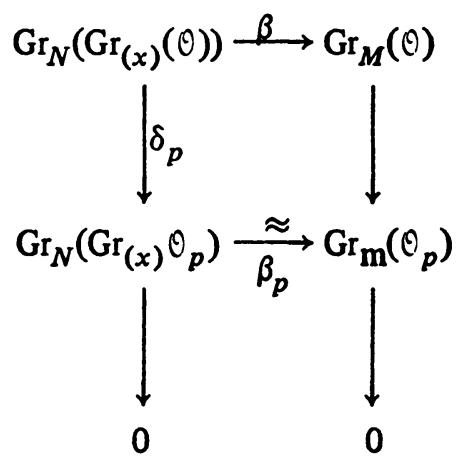

where $\beta_{p}$ is an isomorphism by the remarks above. Since $\mathrm{Gr}_{(x)}\left(\theta_{p}\right)$ is simply the truncation of the graded algebra $\mathrm{Gr}_{(x)}(\theta)$ at level $p$, given any element $\xi \in \mathrm{Gr}_{(x)}(\theta)$ there is a $p$ such that $\delta_{p}(\xi) \neq 0$. The injectivity of $\beta$ follows therefore from that of $\beta_{p}$.

Finally, we must eliminate the artin hypothesis on $A$. For this, let $A$ be arbitrary and now use the notation $\theta_{p}$ to denote $\theta / N^{p} \theta$. With this new interpretation the diagram (2.1.1) is still valid, and the same argument as above works to give the result once we show that given $\xi \neq 0$ $\in \mathrm{Gr}_{N}\left(\mathrm{Gr}_{(x)}(\theta)\right)$, there is a $p$ for which $\delta_{p}(\xi)$ is not zero. Suppose that $\xi$ is homogeneous, of bidegree $\mu, \nu$, so that $\xi$ is the initial form of an element $f$ in $N^{\mu}(x)^{\nu} \theta$. Then $\delta_{p}(\xi)=0$ if and only if $f \in N^{\mu+1}(x)^{\nu} \theta+N^{\mu}(x)^{\nu+1} \theta+N^{p} \theta$. Now since $\theta$ is noetherian,

$$
\bigcap_{p}\left(N^{\mu+1}(x)^{\nu} \theta+N^{\mu}(x)^{\nu+1} \theta+N^{p} \theta\right)=N^{\mu+1}(x)^{\nu} \theta+N^{\mu}(x)^{\nu+1} \theta .
$$

Thus if $\delta_{p}(\xi)=0$ for all $p, \xi=0$, a contadiction. This concludes the proof of (2.1).

(2.2) Definition. Let $R$ be a ring, $I$ and $J$ ideals. An I-standard base of $J$ is a sequence of elements $\left\{f_{i}\right\}_{i}$ of $J$ whose I-adic initial forms $\operatorname{In}_{I}\left(f_{i}\right)$ generate the initial ideal $\mathrm{Gr}_{I}(J, \Re)$ of $J$ in $\mathrm{Gr}_{I}(\Re)$.

We remark that a generating set for $J$ is not necessarily a standard base. For example, if $\Re=k[[x, y, z, w]], I=\max (\Re)$, the elements

$$
f_{1}=x y^{2}+z^{4}, f_{2}=x^{2} y+w^{4}
$$


are not an $I$-standard base for the ideal $J$ which they generate. In fact, if $h=x f_{1}-y f_{2} \in J, \operatorname{In}_{I}(h)=x z^{4}-y w^{4}$, but

$$
\operatorname{In}_{I}(h) \notin\left(\operatorname{In}_{I}\left(f_{1}\right), \operatorname{In}_{I}\left(f_{2}\right)\right) \mathrm{Gr}_{I}(\Re)=\left(x y^{2}, x^{2} y\right) k[x, y, z, w] .
$$

Note that in this example, $J$ is a complete intersection, but $\mathrm{Gr}_{I}(J, \Re)$ is not; $\left(f_{1}, f_{2}, h\right)$ is a minimal standard base.

Conversely, however, it is not hard to show:

(2.3) If $R$ is a local ring, then any I-standard base of $J$ generates $J$ (cf. [8, Chapter II, Lemma 6]).

Recall that if $\Re$ is a ring, $I$ an ideal, $f \in \Re, \nu_{I}(f)$ denotes the highest power of $I$ containing $f$.

(2.4) THEOREM. With the notation of $(2.0), \mathrm{Gr}_{(x)}(\theta)$ is flat over $A$ if and only if there is an $M$-standard base $\left\{f_{i}\right\}$ of $J$ with $\nu_{(x)}\left(f_{i}\right)=\nu_{M}\left(f_{i}\right)$ for each $i$.

Proof. We consider the commutative diagram

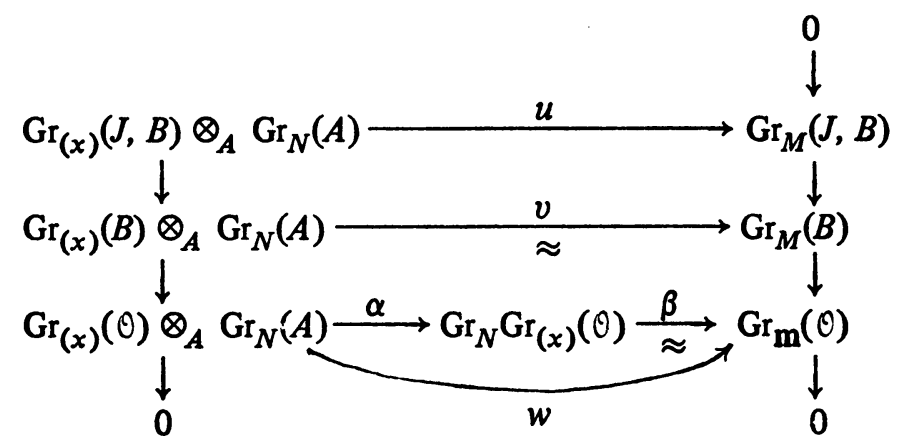

obtained as follows: the vertical sequence on the left arises from applying $\otimes_{A} \mathrm{Gr}_{N}(A)$ to the exact sequence $0 \rightarrow \mathrm{Gr}_{(x)}(J, B) \rightarrow \mathrm{Gr}_{(x)}(B) \rightarrow \mathrm{Gr}_{(x)}(\theta)$ $\rightarrow 0$; the horizontal maps $u, v, w$ are induced by the canonical homomorphisms $\mathrm{Gr}_{(x)}(B) \rightarrow \mathrm{Gr}_{M}(B)$ and $\mathrm{Gr}_{N}(A) \rightarrow \mathrm{Gr}_{M}(B) ; \alpha$ is canonical (and is always surjective), and $\beta$ is the isomorphism of (2.1). We note that $v$ is an isomorphism, since $B$ is just a formal power series ring in $(x)$ over $A$.

By Grothendieck's local criterion of flatness, the flatness of $\mathrm{Gr}_{(x)}(\theta)$ is equivalent to the injectivity of $\alpha$, hence to the injectivity of $w$. By the snake lemma this is equivalent to the surjectivity of $u$. Now the image of $u$ is generated as an ideal by the image of $\operatorname{Gr}_{(x)}(J, B) \otimes_{A} k$. If $\xi$ is an element of $\mathrm{Gr}_{(x)}(J, B) \otimes_{A} k$, say homogeneous of degree $\nu$, then $\xi$ is the class $\bmod N$ of $\operatorname{In}_{(x)}(f)$ for some $f \in J \cap(x)^{\nu}-(x)^{\nu+1}$ and $u(\xi)$ is the image of $f$ in $M^{\nu} / M^{\nu+1}$. Thus it is clear that if $u(\xi) \neq 0, \nu=\nu_{(x)}(f)=\nu_{M}(f)$, so that $u(\xi)$ is the $M$-adic initial form of an element $f \in J$ with this property. Conversely, if $f \in J$ with $\nu_{M}(f)=\nu_{(x)}(f)$, then $\operatorname{In}_{(x)}(f) \notin N \mathrm{Gr}_{(x)}(J, B)$. Hence $\operatorname{In}_{(x)}(f)$ gives a nonzero class $\xi$ in $\operatorname{Gr}_{(x)}(J, B) \otimes_{A} k$ and moreover $u(\xi) \neq 0$ 
(otherwise $\nu_{M}(f)>\nu_{(x)}(f)$ ). We have shown: The image of $u$ is generated by $\left\{\operatorname{In}_{M}(f) \mid f \in J, \nu_{M}(f)=\nu_{(x)}(f)\right\}$. Thus the surjectivity of $u$ is equivalent to the existence of an $M$-standard base of $J$ consisting of such $f$. Q.E.D.

(2.4.2) REMARK. We saw in the proof of (2.4) above that the flatness of $\mathrm{Gr}_{(x)}(\theta)$ is equivalent to the surjectivity of $u$ in the diagram (2.4.1). On the other hand, if $\operatorname{Gr}_{(x)}(\theta)$ is flat then the map $\operatorname{Gr}_{(x)}(J, B) \otimes_{A} \operatorname{Gr}_{N}(A)$ $\rightarrow \mathrm{Gr}_{(x)}(B) \otimes_{A} \mathrm{Gr}_{N}(A)$ is injective, so since $v$ is an isomorphism, so is $u$. Hence, with notation as above, the section $(x)=0$ is normally flat if and only if the natural map $\mathrm{Gr}_{(x)}(J, B) \otimes_{A} \mathrm{Gr}_{N}(A) \stackrel{u}{\longrightarrow} \mathrm{Gr}_{M}(J, B)$ is an isomorphism. It follows that in the normally flat situation, if $\left\{f_{i}\right\}$ is any $(x)$-standard base of $J$ with $\nu_{M}\left(f_{i}\right)=\nu_{(x)}\left(f_{i}\right)$ (or equivalently that $\left.\operatorname{In}_{(x)}\left(f_{i}\right) \notin N \mathrm{Gr}_{(x)}(B)\right)$, then $\left\{f_{i}\right\}$ is also an $M$-standard base.

(2.5) ReMarK. The proof of (2.4) yields the more refined result: $\operatorname{Gr}_{(x)}(\theta)$ is flat over $A$ in degree $\leqslant m$ if and only if there exists an $M$-standard base for $J$ in which those elements $f_{i}$ of $M$-order $\leqslant m$ satisfy $\nu_{(x)}\left(f_{i}\right)=\nu_{M}\left(f_{i}\right)$. Thus, with the terminology of (1.1.2), we have a criterion for the section $x=0$ to be "normally flat of order $n$ ".

(2.6) Suppose we are deforming a hypersurface $X_{0}$ :

$$
\bar{f}=0, \quad \bar{f} \in k\left[\left[x_{1}, \ldots, x_{n}\right]\right] .
$$

Then, with notations as above, $J$ is a principal ideal $(f)$, where $f(\bmod N)=\bar{f}$; $f$ itself is a standard base of $J$. Then we may apply the criterion above (2.5) in the following form:

(2.6.1) The section $(x)=0$ is normally flat of order $m$ if and only if either $\nu_{(x)}(f)=\nu_{M}(f) \leqslant m$, or $\nu_{(x)}(f)>m$.

In fact, if $\nu_{(x)}(f)>m$, then $\nu_{M}(f)>m$, so that there is no standard base in degree $\leqslant m$, and the condition is trivially satisfied.

For example, if $A$ is a $k$-algebra, and $f=f+g$ with $g \in N B$ and $\nu_{(x)}(\bar{f})=\nu$, then if $m \geqslant \nu$ the section $(x)=0$ is normally flat of order $m \Leftrightarrow \nu_{(x)}(g) \geqslant \nu$, i.e. if it is simply normally flat; and if $m<\nu$ the section is normally flat of order $m \Leftrightarrow \nu_{(x)}(g)>m$.

It follows that if we begin with a singular hypersurface $\bar{f}=0$, a section is normally flat of order $1 \Leftrightarrow$ the family "remains singular along the section". Thus in the hypersurface case, in view of the results of $\$ 1$, the functor of normally flat deformations of order 1 has as its prorepresentable hull the "critical locus" of the versal family $\mathfrak{X} \rightarrow S$.

(2.7) EXAMPLE. We consider the space curve $X$ defined by $f_{1}=x y+z^{3}, f_{2}$ $=x^{2}+y^{3} \cdot X$ is a complete intersection, and has an isolated singularity at the origin. However the tangent cone $C$ to $X$ at the origin is not a complete intersection. In fact, if we let $f_{3}=y f_{2}-x f_{1}$, the ideal of $C$ is generated by the initial forms of $f_{1}, f_{2}, f_{3}$, i.e. 


$$
C=\operatorname{Spec} k[x, y, z] /\left(x y, x^{2}, y^{4}-x z^{3}\right) \text {. }
$$

Now a deformation $X^{\prime}$ of $X$ over $k[t] /\left(t^{2}\right)$ is given by an ideal $\left(f_{1}^{\prime}, f_{2}^{\prime}\right)$ in $k[t, x, y, z]$ where $f_{1}^{\prime}=f_{1}+t_{1}, f_{2}^{\prime}=f_{2}+t_{2}$ for any choice of $g_{1}, g_{2}$ in $k[x, y, z]$. Let, for example

(i) $g_{1}=0, g_{2}=z^{2}$. The corresponding deformation is nontrivial, as is seen when we identify these first order deformations with the module

$$
R \oplus R /\left\{\partial f / \partial x, \partial f / \partial y, \partial f / \partial z, f=\left(f_{1}, f_{2}\right)\right\}
$$

via $X^{\prime} \mapsto$ class of $\left(g_{1}, g_{2}\right)$. (Here $\left.R=\Gamma(X)=k[x, y, z] /\left(f_{1}, f_{2}\right)\right)$; we refer to the Jacobian matrix

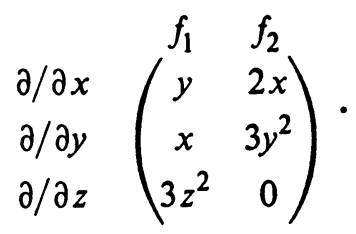

Let $P, M$ denote the ideals $(x, y, z)$ and $(t, x, y, z)$ of $k[t, x, y, z]$ respectively. Note that we have $\nu_{p}\left(f_{i}^{\prime}\right)=\nu_{M}\left(f_{i}^{\prime}\right)=\nu_{P}\left(f_{i}\right)$ for $i=1,2$. However, the deformation is not normally flat along $P=0$, since if we let

$$
f_{3}^{\prime}=y f_{2}^{\prime}-x f_{1}^{\prime}=f_{3}+t\left(y g_{2}-x g_{1}\right)=f_{3}+t y z^{2},
$$

$\operatorname{In}_{P}\left(f_{3}^{\prime}\right)=t y z^{2}$ so that $y z^{2}$ is $t$-torsion in $\operatorname{Gr}_{P}(\mathcal{O})\left(\theta=k[t, x, y, z] /\left(f_{1}^{\prime}, f_{2}^{\prime \prime}\right)\right)$; the point is that here there is no choice $\tilde{g}_{1}, \tilde{g}_{2}$ which give an equivalent deformation and which satisfy $\nu_{P}\left(y \tilde{g}_{2}-x \tilde{g_{1}}\right) \geqslant 4$ (i.e. so that the $P$-adic initial form of the corresponding $f_{3}^{\prime}$ will not be divisible by $t$ ).

By contrast, we consider the deformation given by

(ii) $g_{1}=y^{2}, g_{2}=0$. Here we appear to have the same problem, for $f_{3}^{\prime}=f_{3}-t x y^{2}$, and $\nu_{P}\left(f_{3}^{\prime}\right)=3<\nu_{M}\left(f_{3}^{\prime}\right)$. However in this case the difficulty is only apparent: If we let $x^{\prime}=x+y t$, we get:

$$
f_{1}^{\prime}=f_{1}\left(x^{\prime}, y, z\right), f_{2}^{\prime}=f_{2}\left(x^{\prime}, y, z\right)-2 t x^{\prime} y=f_{2}\left(x^{\prime}, y, z\right)+t\left(2 z^{3}-2 f_{1}^{\prime}\right) .
$$

Thus in terms of the new coordinates $x^{\prime}, y, z$, the ideal defining the deformation is generated by

$$
\tilde{f}_{1}=f_{1}\left(x^{\prime}, y, z\right), \quad \tilde{f}_{2}=f_{2}\left(x^{\prime}, y, z\right)+2 t z^{3} .
$$

Letting $\tilde{f}_{3}=y \tilde{f}_{2}-x \tilde{f}_{1}=f_{3}+2 t y z^{3}$, we find that $\nu_{M}\left(\tilde{f}_{i}\right)=\nu_{\left(x^{\prime}, y, z\right)}\left(\tilde{f}_{i}\right)$ for $i=1,2,3$. Since these $f_{i}$ give a standard base $\bmod (t)$, it then follows from (2.10) below that they are an $M$-standard base. Hence by the criterion of (2.4) above the deformation is normally flat along $x^{\prime}=y=z=0$, which is of course the same as $x=y=z=0$. 
We now consider the question of recognizing a normally flat standard base in the case of a flat family, i.e. (in the terminology (2.0)) when we assume $\theta$ is flat over $A$. The main technical result here is (2.10), which in combination with (2.4) above gives the workable form of the criterion for normally flat deformations. We begin with a few generalities.

Let $R$ be a ring, $I$ and $J$ ideals. Let $P^{\cdot} \rightarrow R / J \rightarrow 0$ be a free resolution; we can apply $\mathrm{Gr}_{I}$ to get a complex which need not be exact for several reasons. However if we use standard bases to construct $P^{\cdot}$ at each stage, and if we suitably "weight" the filtrations of the $P^{\circ}$ then the exactness is preserved. To be precise, suppose that $P^{\cdot}$ has been constructed up to degree $n$ as a complex with "weighted $I$-adic" filtration $\mathfrak{F}^{*}$, so that

$$
P^{n} \stackrel{d_{n}}{\rightarrow} P^{n-1} \rightarrow \cdots \rightarrow P^{0} \rightarrow R / J \rightarrow 0
$$

remains exact upon applying $\mathrm{Gr}_{\mathfrak{F}}$. By such a filtration we mean that for each $i, \mathcal{F}^{i}$ is a filtration of $P^{i}$ such that:

(1) $P^{i}=U_{\nu \in \mathbf{Z}} \mathfrak{F}_{\nu}^{i}$,

(2) $I \mathfrak{F}_{\nu}^{i} \subseteq \mathfrak{F}_{\nu+1}^{i}$, with equality for $\nu$ sufficiently large.

Moreover we require for the filtered complex that for all $i, \nu, d_{i}\left(\mathfrak{F}_{v}^{i}\right) \subset \mathfrak{F}_{v}^{i-1}$; $\mathrm{Gr}_{\mathcal{F}^{\circ}}\left(d_{i}\right)$ then consists of the induced maps $\mathfrak{F}_{\nu}^{i} / \mathfrak{F}_{\nu+1}^{i} \rightarrow \mathfrak{F}_{\nu}^{i-1} / \mathfrak{F}_{\nu+1}^{i-1}$.

This being said, let $K_{n}=\operatorname{ker}\left(d_{n}\right)$, and let $\left\{b_{\alpha}\right\}$ be an $\mathfrak{F}^{n}$-standard base for $K_{n}$ in $P^{n}$, i.e. the $\operatorname{In}_{\mathfrak{F}^{n}}\left(b_{\alpha}\right)$ generate $\mathrm{Gr}_{\mathcal{F}^{n}}\left(K_{n}, P^{n}\right)$ as $\operatorname{Gr}_{I}(R)$-module. Let $\nu_{\alpha}=\nu_{\mathcal{F}^{n}}\left(b_{\alpha}\right)$. Let $P^{n+1}=\oplus_{\alpha} R$, and define $\mathfrak{F}^{n+1}$ by $\mathfrak{F}_{v}^{n+1}=\oplus_{\alpha} I^{\nu-\nu_{\alpha}}\left(I^{p}\right.$ $=R$ if $p \leqslant 0)$. Note that by this definition, if $e_{\alpha}$ is the $\alpha$ th canonical basis element of $P^{n+1}$, then $\nu_{\mathcal{F}^{n+1}}\left(e_{\alpha}\right)=\nu_{\alpha}$. Hence, if $d_{n+1}$ is defined by $e_{\alpha} \mapsto b_{\alpha}$, $\mathrm{Gr}_{\mathcal{F}^{\circ}}\left(d_{n+1}\right)$ sends $\operatorname{In}_{\mathfrak{F}^{n+1}}\left(e_{\alpha}\right)$ to $\operatorname{In}_{\mathfrak{F}^{n}}\left(b_{\alpha}\right)$. Thus $\mathrm{Gr}_{\mathfrak{F}^{\circ}}\left(d_{n+1}\right)$ is surjective onto $\mathrm{Gr}_{\mathfrak{F}^{n}}\left(K_{n}, P^{n}\right)$; and since this latter is the kernel of $\mathrm{Gr}_{\mathfrak{F}}\left(d_{n}\right)$, we get the good construction up to $n+1$.

In particular, we get the following result:

(2.8) If $\left\{f_{1}, \ldots, f_{s}\right\}$ are an I-standard base for $J$, let $\varphi_{1}, \ldots, \varphi_{s}$ be their I-adic initial forms. Let $K \subset R^{s}$ and $\Phi \subset \mathrm{Gr}_{I}(R)^{s}$ be the respective modules of relations. Let $\nu_{i}=\nu_{I}\left(f_{i}\right)$, and let $\mathfrak{F}$ be the filtration of $R^{s}$ defined by $\mathfrak{F}_{\nu}$ $=I^{\nu-v_{1}} \oplus \cdots \oplus I^{\nu-v_{s}}$. View $\mathrm{Gr}_{I}(R)^{s}$ as a graded $\mathrm{Gr}_{I}(R)$ module whose homogeneous part of degree $\nu$ is $\mathrm{Gr}_{I}^{\nu-\nu_{1}}(R) \oplus \cdots \oplus \mathrm{Gr}_{I}^{\nu-\nu_{s}}(R)$. Then $\mathrm{Gr}_{I}(R)^{s}$ and $\mathrm{Gr}_{\mathfrak{F}}\left(R^{s}\right)$ are naturally identified as graded modules, and in this sense $\mathrm{Gr}_{\mathfrak{Y}}\left(K, R^{s}\right)=\Phi$.

Stated more succinctly but less precisely, this says that in the case of a standard base, "module of relations commutes with Gr". It follows that

(2.8.1) If $f_{1}, \ldots, f_{s}$ is an I-standard base of $J$, in a ring $R$, with I-adic initial forms $\varphi_{1}, \ldots, \varphi_{s}$, then any relation among the $\varphi_{i}$ is the $\mathfrak{F}$-adic initial form of $a$ relation among the $f_{i}$, where $\mathfrak{F}$ is the filtration of $R^{s}$ in (2.8) above.

(2.9) Lemma. $R$ a ring, $I, J$ ideals, $f=\left(f_{1}, \ldots, f_{s}\right)$ an I-standard base of $J$. Let 
$r=\left(r_{1}, \ldots, r_{s}\right) \in R^{s}$ with

$$
r \cdot f\left(=\sum_{i=1}^{s} r_{i} f_{i}\right) \in I^{N}
$$

for some integer $N$. Then there is a relation $r^{\prime}=\left(r_{1}^{\prime}, \ldots, r_{s}^{\prime}\right)$ among the $f_{i}(i . e$. $\left.r^{\prime} \cdot f=0\right)$ with

$$
r_{i} \equiv r_{i}^{\prime}\left(\bmod I^{N-v_{i}}\right)
$$

where $\nu_{i}=\nu_{I}\left(f_{i}\right)$.

Proof. Given $r$, let $d(r)=\inf \nu_{I}\left(r_{i} f_{i}\right)$ (note that this is the same as $\nu_{\mathcal{F}}(r)$ where $\mathfrak{F}$ is the filtration of $R^{s}$ defined in (2.8) above). We fix $N$, and prove the lemma by descending induction on $d(r)$.

First observe that if $d(r) \geqslant N$ the condition (*) is trivially verified; and since $r_{i} \in I^{d(r)-v_{i}} \subset I^{N-v_{i}}$ in this case, we get the conclusion by letting $r^{\prime}=0$. Now assume the lemma is true whenever $d(r)\rangle d$, where $d$ is some integer $\langle N$, and suppose we have an $r$ satisfying $(*)$, with $d(r)=d$. Let

$$
r_{(0)}=\left(r_{1(0)}, \ldots, r_{s(0)}\right) \in \mathrm{Gr}_{\mathfrak{F}}\left(R^{s}\right)
$$

denote the $\mathfrak{F}$-adic initial form of $r$. Then, if $\Phi=\left(\varphi_{1}, \ldots, \varphi_{s}\right)$, with $\varphi_{i}$ $=\operatorname{In}_{I}\left(f_{i}\right),(*)$ implies $r_{(0)} \cdot \Phi=0($ since $d<N)$. Hence, by (2.8.1) there is a relation $\bar{r}$ among the $f_{i}$ such that $r_{(0)}=\operatorname{In}_{\mathfrak{F}}(\bar{r})$. In particular $\nu_{\mathcal{F}}(r-\bar{r})$ $=d(r-\bar{r})>d$, and of course we still have $(*)$ for $r-\bar{r}$, i.e.

$$
(r-F) \cdot f \in I^{N} \text {. }
$$

Hence, by induction, there is a relation $\overline{\bar{r}}$ with

$$
\left(r_{i}-\bar{r}_{i}\right) \equiv \bar{r}_{i}\left(\bmod I^{N-v_{i}}\right)
$$

for each $i$. Letting $r^{\prime}=\bar{r}+\bar{r}$, we get the result.

With these preliminaries we can prove the following result on the infinitesimal stability of a standard base in the flat case:

(2.10) Proposition. Let $0 \rightarrow(t) \rightarrow A^{\prime} \rightarrow A \rightarrow 0$ be a "small extension" of local rings, i.e. $\max \left(A^{\prime}\right) t=(0)$. Let $B^{\prime}=A^{\prime}[[x]], B=A[[x]], \bar{B}=k[[x]]$ where $x=\left(x_{1}, \ldots, x_{n}\right)$ and $k$ is the residue field of $A^{\prime}$ (and $\left.A\right)$. Let $M^{\prime}, M, \vec{M}$ denote the maximal ideals of $B^{\prime}, B, \bar{B}$. Let $f_{i}^{\prime}, i=1, \ldots, s$, be elements of $B^{\prime}$; denote their images in $B$ and $\bar{B}$ by $f_{i}$ and $\bar{f}_{i}$. Write $J^{\prime}=\left\{f_{i}^{\prime}\right\} B^{\prime}, J=\left\{f_{i}\right\} B, J$ $=\left\{\bar{f}_{i}\right\} \bar{B}$. 


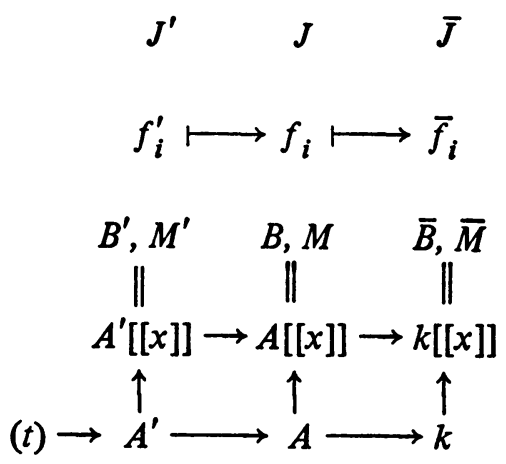

Suppose that

(i) $B^{\prime} / J^{\prime}$ is flat over $A^{\prime}$,

(ii) the $f_{i}$ are an $M$-standard base for $J$.

(iii) the $\bar{f}_{i}$ are an $\bar{M}$-standard base for $\bar{J}$,

(iv) $\nu_{M^{\prime}}\left(f_{i}^{\prime}\right)=\nu_{M}\left(f_{i}\right)$.

Then the $f_{i}^{\prime}$ are an $M^{\prime}$-standard base for $J^{\prime}$.

Proof. Let $u^{(1)}=\left(u_{1}^{(1)}, \ldots, u_{s}^{(1)}\right) \in B^{\prime s}$. We want to show that if $\xi=u^{(1)}$ $\cdot f^{\prime}=\Sigma_{i} u_{i}^{(1)} f_{i}^{\prime}$, then

$$
\operatorname{In}_{M^{\prime}}(\xi) \in\left\{\operatorname{In}_{M^{\prime}}\left(f_{i}^{\prime}\right)\right\} \operatorname{Gr}_{M^{\prime}}\left(B^{\prime}\right)
$$

We first note that

$$
\text { This conclusion holds in case } u^{(1)} \in t B^{\prime s} \text {. }
$$

Namely, $t B^{\prime} \stackrel{\approx}{\approx}$ via $t \mapsto 1$ (small extension), so we conclude by (iii).

Let $\nu_{i}=\nu_{M^{\prime}}\left(f_{i}^{\prime}\right), d_{i}=\nu_{M^{\prime}}\left(u_{i}^{(1)}\right), n_{1}=\inf \left(\nu_{i}+d_{i}\right)$. Thus $\nu_{M^{\prime}}(\xi) \geqslant n_{1}$. Hence if $u^{(1)} \cdot f^{\prime} \notin M^{\prime n_{1}+1}$, we are done, since then

$$
\operatorname{In}_{M^{\prime}}(\xi)=\sum_{i ; \nu_{i}+d_{i}=n_{1}} \operatorname{In}_{M^{\prime}}\left(u_{i}^{(1)}\right) \operatorname{In}_{M^{\prime}}\left(f_{i}^{\prime}\right)
$$

Suppose, then, that $u^{(1)} \cdot f^{\prime} \in M^{\prime n_{1}+1}$. Then by (2.9), since also $\nu_{M}\left(f_{i}\right)=\nu_{i}$ by (iv), we get (letting $\tilde{u}^{(1)}$ denote the image of $u^{(1)}$ in $B^{\mathfrak{s}}$ ):

$$
\tilde{u}^{(1)}=r+\tilde{u}^{(2)}
$$

where $r \cdot f=0$, and $\tilde{u}_{i}^{(2)} \in M^{n_{1}+1-v_{i}}$. Now since $J^{\prime}$ is a flat deformation of $J$ over $A^{\prime}$, we can lift $r$ to a relation $r^{\prime}$ among the $f_{i}^{\prime}$ [5], and we can certainly lift the deviation $\tilde{u}^{(2)}$ to an element $u^{(2)}$ of $B^{\prime s}$ with $u_{i}^{(2)} \in M^{\prime n_{1}+1-v_{i}}$. We then have

$$
u^{(1)}-\left(r^{\prime}+u^{(2)}\right)=v^{(1)} \in t B^{\prime s},
$$

and since $r^{\prime}$ is a relation, 


$$
u^{(1)} \cdot f^{\prime}=v^{(1)} \cdot f^{\prime}+u^{(2)} \cdot f^{\prime} .
$$

Note that the number $n_{2}$ defined for $u^{(2)}$ as $n_{1}$ was for $u^{(1)}$ is strictly larger than $n_{1}$.

Now suppose that after iterating this procedure finitely many times (expressing successive $u^{(j)}$ 's as $v^{(j)}+u^{(j+1)}+$ a relation), we arrive at a $j$ for which

$$
\tilde{u}^{(j)} \cdot f \notin M^{n_{j}+1}
$$

(where, as above, $\tilde{u}^{(j)}$ denotes the image of $u^{(j)}$ in $B^{\mathcal{J}}$ ), so that

$$
u^{(j)} \cdot f^{\prime} \notin M^{\prime n_{j}+1}+(t) \cap M^{\prime n_{j}} .
$$

Then by $(* *), \operatorname{In}_{M^{\prime}}\left(u^{(j)} \cdot f^{\prime}\right)$ is in the ideal generated by the $\operatorname{In}_{M^{\prime}}\left(f_{i}^{\prime}\right)$, and by the above (since it has degree $n_{j}$ ) it is not in $\mathrm{Gr}_{M^{\prime}}\left((t) B^{\prime}, B^{\prime}\right)$.

We now write

$$
\xi=u^{(1)} \cdot f^{\prime}=\left(v^{(1)}+\cdots+v^{(j-1)}\right) \cdot f^{\prime}+u^{(j)} \cdot f^{\prime} .
$$

Let

$$
g=\left(v^{(1)}+\cdots+v^{(j-1)}\right) \cdot f^{\prime} .
$$

Since each $v$ is in $(t) B^{\prime s}, \operatorname{In}_{M^{\prime}}(g)$ is in the ideal generated by the $\operatorname{In}_{M^{\prime}}\left(f_{i}^{\prime}\right)$ by (*), and is of course also in $\mathrm{Gr}_{M^{\prime}}\left((t) B^{\prime}, B^{\prime}\right)$. Hence if $\operatorname{In}_{M^{\prime}}\left(g^{\prime}\right)$ and $\operatorname{In}_{M^{\prime}}\left(u^{(j)} \cdot f^{\prime}\right)$ have the same degree, they cannot cancel each other; thus, in any case, $\operatorname{In}_{M^{\prime}}(\xi)$ is in the ideal generated by the $\operatorname{In}_{M^{\prime}}\left(f_{i}^{\prime}\right)$ as desired.

We must finally consider the case where the iteration procedure does not terminate, i.e. when there is no $j$ for which $(* * *)$ holds. Then

$$
\xi=u^{(1)} \cdot f=\lim _{j \rightarrow \infty} \sum_{m=1}^{j}\left(v^{(m)} \cdot f^{\prime}\right) .
$$

In fact, the limit makes sense because

$$
u^{(1)} \cdot f^{\prime}-\left(v^{(1)}+\cdots+v^{(j)}\right) \cdot f^{\prime} \in M^{\prime n_{j}},
$$

and $n_{j} \rightarrow \infty$ as $j \rightarrow \infty$. Thus in this case, we may assume $u^{(1)} \in t B^{\prime s}$, so we conclude by (*). Q.E.D.

(2.11) Remark. In Proposition (2.10) it would suffice to assume merely that the $f_{i}^{\prime}$ are in $J^{\prime}$; that they then generate $J^{\prime}$ follows from the flatness, and the assumption that they generate $\bmod (t)$. Namely, in view of the flatness, $t B^{\prime} \cap J^{\prime}=t J^{\prime}$. Hence, since the $f_{i}^{\prime}$ generate $\bmod (t)$,

$$
J=\left\{f_{i}^{\prime}\right\} B+t J=\left\{f_{i}^{\prime}\right\} B+t\left\{f_{i}^{\prime}\right\} B+t^{2} J=\left\{f_{i}^{\prime}\right\} B
$$

since $t^{2}=0$. 
(2.12) REMARK. We give an example which shows that condition (iii) in the hypothesis of Proposition (2.10) above is necessary, i.e. that (i), (ii), (iv) do not by themselves imply that the $f_{i}$ are an $M^{\prime}$-standard base:

Let $A=k[[\tau]], A^{\prime}=k[[\varepsilon, \tau]] /\left(\varepsilon^{2}, \varepsilon \tau\right)$ (so that in the terminology of $(2.10)$, $t=\varepsilon)$. Let

$$
f_{1}=x^{2} y+\tau x^{4}+w^{6}, f_{2}=x y^{2}
$$

in $A[[x, y, w]]=B$. We first note that $f_{1}, f_{2}$ are an $M$-standard base of the ideal they generate. To see this, observe that the only relations among the initial forms of $f_{1}, f_{2}$ (other than the "trivial" relations) are the multiples of $(y,-x)$. Now $y f_{1}-x f_{2}=f_{3}=\tau y x^{4}+y w^{6}$, so $\operatorname{In}_{M}\left(f_{3}\right)=\tau y x^{4}=\tau x^{2} \operatorname{In}_{M}\left(f_{1}\right)$. Moreover, $\tau y x^{4}$ is not a zero-divisor in $B$, so any multiple of $(y,-x)$ still yields an element whose initial form is a multiple of $\operatorname{In}_{M}\left(f_{1}\right)$. (However $y \bar{f}_{1}-x \bar{f}_{2}$ $=y w^{6}$, so we fail to have (iii) in this situation.)

Now let $f_{1}^{\prime}$ and $f_{2}^{\prime}$ be the elements of $B^{\prime}=A^{\prime}[[x, y, w]]$ which are written the same way as $f_{1}, f_{2}$. Since $\varepsilon$ kills $\tau, \varepsilon y f_{1}-\varepsilon x f_{2}=\varepsilon y w^{6}$, which is not in the ideal generated by $\operatorname{In}_{M^{\prime}}\left(f_{1}^{\prime}\right), \operatorname{In}_{M^{\prime}}\left(f_{2}^{\prime}\right)$, i.e., $f_{1}^{\prime}, f_{2}^{\prime}$ are not an $M^{\prime}$-standard base although (i), (ii), (iv) are satisfied.

For the above example it was crucial that $\tau$ not be a zero divisor in $A$; in particular, the example would not have worked if we let $A=k[[\tau]] / \tau^{2}$ instead of $k[[\tau]]$. We can, however, get an example in this case as follows: Let

$$
A=k[[\tau]] / \tau^{2}, \quad A^{\prime}=k[[\varepsilon, \tau]] /\left(\varepsilon^{2}, \varepsilon \tau, \tau^{2}\right) .
$$

Let $B^{\prime}$ and $B$ be $A^{\prime}[[x, y, z, w]], A[[x, y, z, w]]$ respectively. Let

$$
f_{1}=x^{2} y+\tau x^{4}+w^{6}, f_{2}=x y^{2}, \quad f_{3}=\tau y w^{6}+z^{10}
$$

in $B$, and let the $f_{i}^{\prime}$ be the elements of $B^{\prime}$ which are written the same way. The point here is that we have chosen $f_{3}$ so that $\tau y f_{1}-\tau x f_{2}$ has the same initial form as $f_{3}$ (whereas $y f_{1}-x f_{2}$ has initial form $\tau x^{2} \operatorname{In}_{M}\left(f_{1}\right)$ as in the previous example). Thus $f_{1}, f_{2}, f_{3}$ are a standard base. However, $\operatorname{In}_{M^{\prime}}\left(\varepsilon y f_{1}^{\prime}-\varepsilon x f_{2}^{\prime}\right)$ $=\varepsilon y w^{6}$, which cannot be expressed in terms of the $\operatorname{In}_{M^{\prime}}\left(f_{i}^{\prime}\right)$. Note that the $\bar{f}_{i}$ fail to be an $\bar{M}$-standard base (again $y \bar{f}_{1}-x \bar{f}_{2}$ is bad) but they are a complete intersection, so that everything is flat; again (i), (ii), (iv) hold but the conclusion of (2.10) is false. This example is of interest for the additional reason that the extension $B^{\prime} / J^{\prime} \rightarrow B / J$ is split (where $J^{\prime}, J$ are the ideals generated by the $f_{i}^{\prime}$ and the $f_{i}$ ) over the obvious splitting of $A^{\prime} \rightarrow A$; in fact we have a commutative diagram:

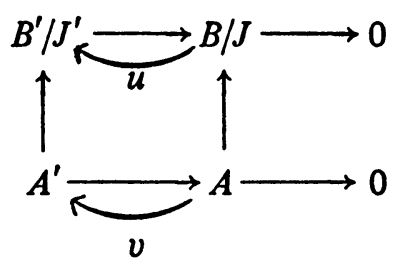


where $u, v$ are $k$-algebra homomorphisms. We see that the standard base property is unstable even under this type of "trivial" deformation.

This strange phenomenon is irrelevant to our application, where (iii) is given as part of the basic setup:

(2.13) THEOREM. Let $A$ be a local ring, and let $\bar{f}_{1}, \ldots, \bar{f}_{s}$ be an $(x)$-standard base of an ideal $J$ in $k[[x]]$, where $x=\left(x_{1}, \ldots, x_{n}\right)$ and $k$ is the residue field of $A$. Suppose $J \subset A[[x]]$ is a flat deformation. Then the deformation is normally flat along $(x)=0$ if and only if there exist elements $f_{1}, \ldots, f_{s}$ of $J$ with $f_{i} \mapsto \bar{f}_{i}$ and $\nu_{(x)}\left(f_{i}\right)=\nu_{(x)}\left(\bar{f}_{i}\right)(i=1, \ldots, s)$; these $f_{i}$ are then both an $M$-standard base and an $(x)$-standard base of $J(M=\max A[[x]])$.

Proof. We prove the "if" part first:

Let $B=A[[x]]$. Since for any $f \in B$ we have inequalities $\nu_{(x)}(f) \leqslant \nu_{M}(f)$ $\leqslant \nu_{(x)}(\bar{f})$ (where - denotes reduction $\bmod \max (A)$ ), the hypotheses imply that $\nu_{(x)}\left(f_{i}\right)=\nu_{M}\left(f_{i}\right), i=1, \ldots, s$. Thus if we can show that the $f_{i}$ are an $M$ standard base for $J$, the conclusion will follow from Theorem (2.4). Let $N=\max (A)$, and for every integer $l$, let $B_{l}, M_{l}, J_{l}$ denote the reductions $\bmod N^{l}$. We first claim that if the images of the $f_{i}$ in $B_{l}$ are an $M_{r}$ standard base of $J_{l}$ for each $l$, then the $f_{i}$ are an $M$-standard base for $J$ (this reduces the theorem to the case when $A$ is an artin ring).

To see this, let $f \in J$. Let $\nu=\nu_{M}(f)$, and let $l>\nu$. Then if $\sim$ denotes reduction $\bmod N^{l}, \operatorname{In}_{\tilde{M}}(\tilde{f})$ is the image of $\operatorname{In}_{M}(f)$ in $\operatorname{Gr}_{M_{l}}\left(B_{l}\right)$ $\left(=\mathrm{Gr}_{M}(B) / \mathrm{Gr}_{M}\left(N^{l} B, B\right)\right)$. Hence, assuming the $f_{i}$ are an $M_{\Gamma}$ standard base, we get

$$
\operatorname{In}_{M}(f) \in\left\{\operatorname{In}_{M}\left(f_{i}\right)\right\} \operatorname{Gr}_{M}(B)\left(\bmod \mathrm{Gr}_{M}\left(N^{l} B, B\right)\right) .
$$

But since $\mathrm{Gr}_{M}\left(N^{l} B, B\right) \cap \mathrm{Gr}_{M}^{\nu}(B)=(0)($ since $l>\nu)$, we find that $\operatorname{In}_{M}(f)$ $\in\left\{\operatorname{In}_{M}\left(f_{i}\right)\right\} \operatorname{Gr}_{M}(B)$.

We now prove the "if" part of the theorem when $A$ is artin. Write

$$
A=A_{n} \rightarrow A_{n-1} \rightarrow \cdots \rightarrow A_{1} \rightarrow A_{0}=k
$$

where each step is a small extension. Let $M_{j}=\max \left(B_{j}\right), B_{j}=A_{j}[[x]]$, and let $f_{i}^{(j)}$ denote the image of $f_{i}$ in $B_{j}$ (so $f_{i}^{(n)}=f_{i}, f_{i}^{(0)}=\bar{f}_{i}$ ). Now for each $j$, we have inequalities:

$$
\begin{aligned}
\nu_{(x)}\left(f_{i}^{(j)}\right) & \leqslant \nu_{M_{j}}\left(f_{i}^{(j)}\right) \leqslant \nu_{M_{0}}\left(f_{i}^{(0)}\right)=\nu_{(x)}\left(\bar{f}_{i}\right) . \\
\mathrm{V} / & v_{(x)}\left(f_{i}\right) \leqslant \nu_{M}\left(f_{i}\right) .
\end{aligned}
$$

Thus, the hypothesis $\nu_{(x)}\left(f_{i}\right)=\nu_{(x)}\left(\bar{f}_{i}\right)$ implies

(1) For all $j, \nu_{(x)}\left(f_{i}^{(j)}\right)=\nu_{M_{j}}\left(f_{i}^{(j)}\right)$,

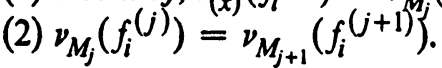


By (2), applying Proposition (2.10) to each step (noting Remark (2.11)), we get by induction: the $\left\{f_{i}\right\}$ are an $M$-standard base of $J$ as desired. We conclude by (2.4) as noted above.

Now for the "only if" part: Choose $g_{1}, \ldots, g_{s} \in J$ with $g_{i} \mapsto \bar{f}_{i}$. If $\nu_{(x)}\left(g_{i}\right)=\nu_{(x)}\left(\bar{f}_{i}\right)$, then we are done, letting $g_{i}=f_{i}$. If $\nu_{(x)}\left(g_{i}\right)<\nu_{(x)}\left(\bar{f}_{i}\right)$, then we must have $\operatorname{In}_{(x)}\left(g_{i}\right) \in N \mathrm{Gr}_{(x)}(B)$. Now in view of the normal flatness (which asserts that $\mathrm{Gr}_{(x)}(B) / \mathrm{Gr}_{(x)}(J, B)$ is flat over $A$ ), we have

$$
N \mathrm{Gr}_{(x)}(B) \cap \mathrm{Gr}_{(x)}(J, B)=N \mathrm{Gr}_{(x)}(J, B) .
$$

Hence $\operatorname{In}_{(x)}\left(g_{i}\right)=\sum_{j} t_{j} \operatorname{In}_{(x)}\left(h_{j}\right)$, with $h_{j} \in J, t_{j} \in N$. Let $h=\sum_{j} t_{j} h_{j}$ $\in N B$, and let $g_{i}^{(1)}=g_{i}-h$. Then we still have $g_{i}^{(1)} \mapsto \bar{f}_{i}$, and $\nu_{(x)}\left(g_{i}^{(1)}\right)$ $>\nu_{(x)}\left(g_{j}\right)$. After finitely many repetitions of this alternation process, we arrive at a $g_{i}^{(d)}$, for some $d$, with $\nu_{(x)}\left(g_{i}^{(d)}\right)=\nu_{(x)}\left(\bar{f}_{i}\right)$, and of course $g_{i}^{(d)} \mapsto \bar{f}_{i}$. Letting $f_{i}=g_{i}^{(d)}$, we are done.

Finally, to see that these $f_{i}$ are both an $(x)$ and an $M$-standard base of $J$, we first note that by the normal flatness

$$
\mathrm{Gr}_{(x)}(J, B) \otimes_{A} A / N \stackrel{\approx}{\approx} \mathrm{Gr}_{(x)}(\bar{J}, \bar{B})
$$

Since this isomorphism makes the class of $\operatorname{In}_{(x)}\left(f_{i}\right)$ correspond to that of $\operatorname{In}_{(x)}\left(\bar{f}_{i}\right)\left(\right.$ since $\left.\nu_{(x)}\left(f_{i}\right)=\nu_{(x)}\left(\bar{f}_{i}\right)\right)$, we find by Nakayama that the $f_{i}$ are an $(x)$ standard base of $J$. That the $f_{i}$ are an $M$-standard base then follows from the isomorphism

$$
\mathrm{Gr}_{M}(J, B)=\mathrm{Gr}_{(x)}(J, B) \otimes_{A} \operatorname{Gr}_{N}(A)
$$

which holds in the normally flat situation (see (2.4.2)). This completes the proof of (2.13).

(2.14) REMARK. An easy modification of (2.13) gives the criterion for normally flat deformations of order $j$ :

Theorem. Let $A, \bar{f}_{1}, \ldots, \bar{f}_{s}, \bar{J}, J$ be as in (2.13). Then the family $A[X] / J$ is normally flat of order $j$ if and only if there exist elements $f_{1}, \ldots, f_{s}$ in $J$ with $f_{i} \mapsto \bar{f}_{i}$ and

(i) $\nu_{(x)}\left(f_{i}\right)=\nu_{(x)}\left(\bar{f}_{i}\right)$ if $\nu_{(x)}\left(\bar{f}_{i}\right) \leqslant j$,

(ii) $\nu_{(x)}\left(f_{i}\right)>j$ if $\nu_{(x)}\left(\bar{f}_{i}\right)>j$.

Then those $f_{i}$ with $\nu_{(x)}\left(f_{i}\right) \leqslant j$ are both an $M$-standard base and an $(x)$-standard base for $J$ in degree $\leqslant j$.

Proof. Simply replace $J$ by $J+(x)^{j+1}$. Condition (ii) is equivalent to the assertion that $J+(x)^{j+1}$ is generated in degree $>j$ by the monomials of degree $j+1$ in $(x)$. Note that this is true of $\bar{J}+(x)^{j+1}$ in any case, and the degree of such a monomial is invariant under reduction mod the maximal 
ideal of $A$. Hence conditions (i) and (ii) together are equivalent to the normal flatness of $A[x] / J+(x)^{j+1}$ by $(2.13)$; and this is the same as the normal flatness of $A[x] / J$ of order $j$.

We now apply these results to that class of singularities which are the "nicest possible" from the standpoint of normally flat deformation theory:

(2.15) Definition. A local ring $\theta$ is called a "strict complete intersection" if $\mathrm{Gr}_{\mathrm{m}}(\theta)$ is a complete intersection, $\mathrm{m}=\max (\theta)$. Thus if $\theta=R / J$, where $R$ is a regular local ring with maximal ideal $M$, then $\theta$ is a strict complete intersection if and only if $\mathrm{Gr}_{M}(J, R)$ is generated by a regular sequence in $\mathrm{Gr}_{M}(R)$. A strict complete intersection is always a complete intersection (via (2.3)); the converse is false, as any of the examples in this section show.

We recall (1.4.3) that given any singularity $\theta, m$ there is a natural morphism $\mathbf{u}$ from the normally flat deformation functor of $\theta$ to the cone-deformation functor of $\mathrm{Gr}_{\mathrm{m}}(\theta)$ : given a normally flat deformation $X$ of $\theta$ over $A$, with section $\sigma, u(X, \sigma)==_{\text {def }} \mathrm{Gr}_{\sigma}(X)$, viewed as a deformation of $\mathrm{Gr}_{\mathrm{m}}(\theta)$.

(2.16) THEOREM. If $O$ is a strict complete intersection, $u$ is a smooth morphism.

PROof. The assertion means that the map of formal moduli induced by $u$ is a smooth morphism or equivalently, that the normally flat deformations are "relatively unobstructed" over the cone deformations. We must show that if $(X, \sigma)$ is a normally flat deformation of $\theta$ over $A$, with $\operatorname{Spec}\left(\mathrm{Gr}_{\sigma}(X)\right)=C$, and if $A^{\prime} \rightarrow A$ is an extension with a cone deformation $C^{\prime}$ over $A^{\prime}$ inducing $C$, then there is a normally flat deformation $\left(X^{\prime}, \sigma^{\prime}\right)$ over $A^{\prime}$ inducing $(X, \sigma)$ over $A$ and $\operatorname{Spec}\left(\mathrm{Gr}_{\left(\sigma^{\prime}\right)}\left(X^{\prime}\right)\right)=C^{\prime}$.

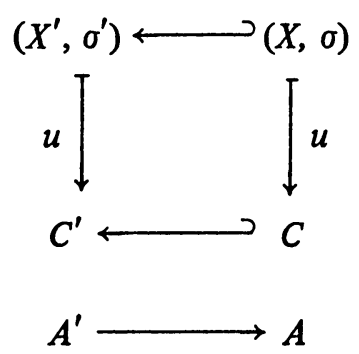

To see this, write $\theta=\bar{B} / \bar{J}, \bar{B}=k[[x]]$, and choose an $(x)$-standard base $\bar{f}_{1}, \ldots, \bar{f}_{s}$ whose initial forms $\bar{\varphi}_{1}, \ldots, \bar{\varphi}_{s}$ are a regular sequence in $\mathrm{Gr}_{(x)}(\bar{B})$. (Then $\bar{f}_{1}, \ldots, \bar{f}_{s}$ are a regular sequence in $\bar{B}$.) By a change of coordinate, we can assume the section $\sigma$ in the normally flat deformation $X$ over $A$ is $(x)=0$, where $X=\operatorname{Spec}(B / J), B=A[[x]]$. Then by Theorem (2.13),

$$
J=\left(f_{1}, \ldots, f_{s}\right) b \quad \text { where } f_{i} \mapsto \bar{f}_{i}, \nu_{(x)}\left(f_{i}\right)=\nu_{i}=\nu_{(x)}\left(\bar{f}_{i}\right),
$$

and the $f_{i}$ are an $(x)$-standard base. Hence if $\varphi_{i}=\operatorname{In}_{(x)}\left(f_{i}\right), \varphi_{i} \mapsto \bar{\varphi}_{i}$ and the cone deformation $C$ is $\operatorname{Spec}\left(A[x] /\left(\varphi_{1}, \ldots, \varphi_{s}\right)\right)$; then $C^{\prime}$ is given by 
$A^{\prime}[x] /\left(\varphi_{1}^{\prime}, \ldots, \varphi_{s}^{\prime}\right)$ where the $\varphi_{i}^{\prime}$ are forms of degree $\nu_{i}$ in $(x)$ and $\varphi_{i}^{\prime} \mapsto \varphi_{i}$.

Now to get $X^{\prime}$, first lift the $f_{i}$ to elements $g_{i}$ in $A^{\prime}[[x]]$ so that $\nu_{(x)}\left(g_{i}^{\prime}\right)$ $=\nu_{(x)}\left(f_{i}\right)$. Let $\eta_{i}=\operatorname{In}_{(x)}\left(g_{i}\right)$. Then $\eta_{i} \mapsto \varphi_{i}$, so $\eta_{i}-\varphi_{i}^{\prime}=\xi_{i}$, another form of degree $\nu_{i}$ in $(x)$, and $\xi_{i} \mapsto 0$ in $A[x]$. Then let $f_{i}^{\prime}=g_{i}^{\prime}-\xi_{i}$ (where we now view $\xi_{i}$ in $A^{\prime}[[x]]$ instead of $\left.A^{\prime}[x]\right)$. Thus $\operatorname{In}_{(x)}\left(f_{i}^{\prime}\right)=\varphi_{i}^{\prime}, f_{i}^{\prime} \mapsto f_{i}$, and of course we still have $\nu_{i}=\nu_{(x)}\left(f_{i}^{\prime}\right)=\nu_{(x)}\left(\bar{f}_{i}\right)$. Moreover, if $J^{\prime}=\left(f_{1}^{\prime}, \ldots, f_{s}^{\prime}\right) B^{\prime}\left(B^{\prime}\right.$ $\left.=A^{\prime}[[x]]\right)$, then $B^{\prime} / J^{\prime}$ is flat, since the $\left\{f_{i}^{\prime}\right\}$ yield the regular sequence $\left\{\bar{f}_{i}\right\}$ at the closed fibre. Hence we can apply (2.13) to deduce that $X^{\prime}=\operatorname{Spec}\left(B^{\prime} / J^{\prime}\right)$ is normally flat along $\sigma^{\prime}:(x)=0$ and the $f_{i}^{\prime}$ are an $(x)$-standard base. Therefore, since $\operatorname{In}_{\left(x^{\prime}\right)}\left(f_{i}^{\prime}\right)=\varphi_{i}^{\prime}$ by construction, $\operatorname{Spec}\left(\mathrm{Gr}_{\mathrm{o}^{\prime}}\left(X^{\prime}\right)\right)=C^{\prime}$, and we are done.

(2.17) THEOREM. The normally flat deformations of a strict complete intersection are unobstructed.

Proof. Here, given $A^{\prime} \rightarrow A$ and a normally flat deformation over $A$, we must simply lift it to one over $A^{\prime}$. Thus the proof is the same as above, except it is easier since we need not worry about compatibility with a $C^{\prime}$ given beforehand. (Alternatively we can get this as a corollary of (2.16), noting that the cone-deformations of a complete intersection are unobstructed.)

Similarly, noting the remark (2.14), the evident modification of the direct proof of (2.17) yields the generalization:

(2.18) For any $j$, the normally flat deformations of order $j$ of a strict complete intersection are unobstructed.

Note that in view of (1.2.4) (and with the terminology of $\$ 1),(2.18)$ is equivalent to the smoothness of the $N_{j}$. This was proved in the special case of plane curves for $j=\infty$ (i.e. for the equimultiple deformations) by J. Wahl [10], and for arbitrary $j$ in the case of hypersurfaces in characteristic 0 by B. Teissier $[9, \mathrm{I}]$.

\section{An example.}

(3.0) We now want to work out in detail one nontrivial example in which the normally flat deformations, the cone deformations, and the morphism $\mathbf{u}$ between them are computable. The singularity in question is the space-curve $X$ defined by the equations

$$
f_{1}=x^{2} y^{2}+z^{5}+x^{6}, \quad f_{2}=x^{2} z^{2}+y^{5} .
$$

Thus $X$ is a complete intersection, and its only singularity is at the origin, $P$. Let $J=\left(f_{1}, f_{2}\right) k[x, y, z]$ be the ideal of $X$ in $\mathbf{A}^{3}$, and let $M$ denote the maximal ideal of the origin in $A^{3}$. To get an $M$-standard base of $J$ we need to add the polynomial

$$
f_{3}=z^{2} f_{1}-y^{2} f_{2}=z^{7}-y^{7}+x^{6} z^{2}
$$


so that $X$ is not a strict complete intersection. We will find, however, that the morphism $\mathbf{u}$ is smooth (compare Theorem (2.16)), and that both the normally flat deformations of $X$ and the cone deformations of the tangent cone to $X$ at $P$ are (separately) unobstructed. Thus in these respects the singularity behaves like a strict complete intersection. However as the example shows, this fact lies fairly deep in its specific properties, and does not follow easily from general theory we have developed thus far.

I originally worked out the example in the hope that it might give a negative answer to the question: Is the morphism u smooth for arbitrary singularities? Nevertheless, this question remains open, and indeed the reader may find that the example suggests evidence of an affirmative answer!

(3.1) Now let $C$ denote the tangent cone to $X$ at the origin. Then $C$ is defined by the initial forms $\varphi_{i}$ of the $f_{i}(i=1,2,3)$ :

$$
\varphi_{1}=x^{2} y^{2}, \quad \varphi_{2}=x^{2} z^{2}, \quad \varphi_{3}=z^{7}-y^{7} .
$$

We will first study the cone-deformations of $C$. Over $k[\varepsilon] / \varepsilon^{2}$ these are given by equations $\varphi_{i}^{\prime}+\varepsilon h_{i}(i=1,2,3)$ where $h_{i}$ is a form in $x, y, z$ of the same degree as $\varphi_{i}$, and moreover the flatness condition is satisfied: If $g$ denotes the ideal generated by the $\varphi_{i}$, and if $r=\left(r_{1}, r_{2}, r_{3}\right)$ is a relation among the $\varphi_{i}$ (i.e. $\left.r \cdot \varphi=0, \varphi=\left(\varphi_{1}, \varphi_{2}, \varphi_{3}\right)\right)$, then $\left.r \cdot h \in g\left(h=h_{1}, h_{2}, h_{3}\right)\right)$. In other words the condition is

(3.1.1) For each relation $r$ among the $\varphi_{i}$, there exists $a_{i}(i=1,2,3)$ in $k[x, y, z]$ such that, if we denote $a=\left(a_{1}, a_{2}, a_{3}\right)$,

$$
-r \cdot h=a \cdot \varphi
$$

(the negative sign is for our later convenience).

More generally, suppose we have a flat family over $k[\varepsilon] / \varepsilon^{n+1}$, say given by

$$
\varphi_{(n)}=\varphi+\varepsilon h_{(1)}+\cdots+\varepsilon^{n} h_{(n)}
$$

(where $\varphi_{(n)}, \varphi, h_{(j)}$ denote 3-tuples). Every relation $r$ among the $\varphi_{i}$ extends to a relation $\tilde{r}$ among the $\varphi_{n(i)}$ of the form

$$
\tilde{r}=r+\varepsilon a_{(1)}+\cdots+\varepsilon^{n} a_{(n)} .
$$

It is easily checked that the condition for $\varphi_{(n)}$ to extend to a flat family over $k[\varepsilon] / \varepsilon^{n+2}$ is as follows:

(3.1.2) There exists $h_{(n+1)}$ such that for every relation $\tilde{r}$ among the $\varphi_{(n)}$ as above

$$
-r \cdot h_{(n+1)} \equiv a_{(1)} h_{(n)}+a_{(2)} h_{(n-1)}+\cdots+a_{(n)} h_{(1)}(\bmod g) .
$$

In fact if this is so, we can let 


$$
\varphi_{(n+1)}=\varphi_{(n)}+\varepsilon^{n+1} h_{(n+1)},
$$

and if the two sides of the congruence above differ by say $a_{(n+1)} \cdot \varphi$, then $\tilde{r}$ extends to the relation $\tilde{r}+\varepsilon^{n+1} a_{(n+1)}$ among the $\varphi_{(n+1) i} \bmod \varepsilon^{n+2}$.

(3.2) Remarks. (1) Note that (3.1.1) is just the case $n=1$ of (3.1.2), but it is much more convenient to write this case out explicitly without the subscripts.

(2) The obstruction to extending a family from $n=1$ to $n=2$ is called a "primary obstruction". Here it is also convenient to write down the condition without subscripts: the family $\varphi^{\prime}=\varphi+\varepsilon h$ over $k[\varepsilon] / \varepsilon^{2}$ extends over $k[\varepsilon] / \varepsilon^{3}$ if and only if there exists $\tilde{h}$ such that, for every relation $r$ among the $\varphi_{i}$, if $r \cdot h=a \cdot \varphi$, then

$$
a \cdot h \equiv r \cdot \tilde{h}(\bmod g) .
$$

(3) Our description of the conditions for extending these families is not a priori canonical, since the $a_{(i)}$ are not uniquely determined. However it is not hard to show that the condition is independent of the choice of the $a_{(i)}$, i.e. of the choice of the extension $\tilde{r}$ of $r$. For details, and in general for a complete and more functorial treatment of the obstruction theory, the reader is referred to [5].

(4) We will see below that every cone deformation of $C$ over $k[\varepsilon] / \varepsilon^{2}$ extends to a family over $k[[\varepsilon]]$. In other words, every tangent vector is represented by a formal nonsingular curve in the moduli. This is sufficient to give the unobstructedness of the deformation theory (although in the general case the investigation of this question is insufficient for a complete understanding of the obstructions). This fact is essentially the same as the following.

(3.2.1) Lemma. Suppose $R, M$ is a complete local $k$-algebra, with say $R / M=k$, and with the property: Every $k$-algebra homomorphism $R \rightarrow k[\varepsilon] / \varepsilon^{2}$ extends to $k[[\varepsilon]]$. Then $R$ is a formal power series ring over $k$.

Proof. Choose representations $x_{1}, \ldots, x_{n}$ in $M$ of a $k$-basis of $M / M^{2}$, so that $R=k\left[\left[x_{1}, \ldots, x_{n}\right]\right] / I$ for some ideal $I$. We want to show $I=(0)$. If $I \neq(0)$, let $f$ be an element of $I$ of minimal order $\nu$ (as a power series in $\left.x_{1}, \ldots, x_{n}\right) ; \nu$ is at least 2. Let $\varphi$ denote the initial form of $f$, so that $\varphi$ is a form of degree $\nu$. Choose $a_{1}, \ldots, a_{n} \in k$ so that $\varphi\left(a_{1}, \ldots, a_{n}\right) \in k\left({ }^{9}\right)$. Now since $I \subset\left(x_{1}, \ldots, x_{n}\right)^{\nu}$, the map $k\left[\left[x_{1}, \ldots, x_{n}\right]\right] \rightarrow k[\varepsilon] / \varepsilon^{\nu}$ defined by $x_{i} \mapsto a_{i} \varepsilon$ factors through $R$, say

$$
\rho: R \rightarrow k[\varepsilon] / \varepsilon^{\nu}, \quad x_{i} \mapsto a_{i} \varepsilon
$$

${ }^{(9}$ )Since base field extension $k \rightarrow L$ preserves the hypothesis and reflects the conclusion, we may assume $k$ is infinite. 
But clearly $\rho$ cannot be factored through $k[\varepsilon] / \varepsilon^{\nu+1}$, since if $\rho^{\prime}$ were such a factor, $\rho^{\prime}(f(x))$ would have to be $\varphi\left(a_{1}, \ldots, a_{n}\right) \varepsilon^{\nu} \neq 0$ in $k[\varepsilon] / \varepsilon^{\nu+1}$. This contradiction shows that $I=(0)$. Q.E.D.

(5) Since we are concerned with cone deformations, we require that at each stage (i.e. for each $n$ as in (3.1.2)), the $h_{(n) i}$ are homogeneous, of the same degree as $\varphi^{\prime}$. If we drop this requirement then (3.1.2) is the condition for extending a deformation of an arbitrary singularity defined by some ideal $\left\{\varphi_{i}\right\}$.

(3.3) With these preliminaries, we now compute the cone moduli of our tangent cone $C: \varphi_{1}=\varphi_{2}=\varphi_{3}=0$, where $\varphi_{1}=x^{2} y^{2}, \varphi_{2}=x^{2} z^{2}, \varphi_{3}=z^{7}$ $-y^{7}$. We denote by $g$ the ideal generated by the $\varphi_{i}$. One observes that the condition (3.1.2) is trivially satisfied for a relation $r=\left(r_{1}, r_{2}, r_{3}\right)$ with the $r_{i} \in g$. Thus in our case it is easy to check that there are only two significant relations, namely

$$
r^{(1)}=\left(z^{2},-y^{2}, 0\right) \text { and } r^{(2)}=\left(-y^{5}, z^{5},-x^{2}\right) .
$$

Then according to (3.1.1) the conditions that certain homogeneous polynomials $h_{1}, h_{2}, h_{3}$ (of degrees $4,4,7$ ) give a flat family over $k[\varepsilon] / \varepsilon^{2}$ are

$$
\begin{array}{ll}
z^{2} h_{1}-y^{2} h_{2} \in g & \left(\text { from } r^{(1)}\right), \\
-y^{5} h_{1}+z^{5} h_{2}-x^{2} h_{3} \in g & \text { (from } \left.r^{(2)}\right) .
\end{array}
$$

Since the only generators of $g$ in degree $\leqslant 6$ are $z^{2} x$ and $y^{2} x^{2}$, (1) implies that

$$
h_{1}=x^{2} u, \quad h_{2}=x^{2} v
$$

for some $u, v$ of degree 2 . We get, letting $h=\left(h_{1}, h_{2}, h_{3}\right)$

$$
r^{(1)} \cdot h=-v \varphi_{1}+u \varphi_{2} \text {. }
$$

In other words, the " $a$ " works for $r^{(1)}$ as in (3.1.1) is $a^{(1)}=(v,-u, 0)$. Note that

$$
a^{(1)} \cdot h=(v,-u, 0)\left(x^{2} u, x^{2} v, h_{3}\right)=0 .
$$

Now in view of what we know about $h_{1}, h_{2}$, equation (2) above becomes

$$
-y^{5} x^{2} u+z^{5} x^{2} v-x^{2} h_{3} \in g,
$$

i.e. simply $x^{2} h_{3} \in g$, which is equivalent to saying that $h_{3} \in\left(z^{2}, y^{2}\right) k[x, y, z]$. Hence, for some $w_{1}, w_{2}$ of degree 5 ,

$$
h_{3}=y^{2} w_{1}+z^{2} w_{2} \text {. }
$$

We get 


$$
r^{(2)} \cdot h=\left(-y^{3} u-w_{1}\right) \varphi_{1}+\left(z^{3} v-w_{2}\right) \varphi_{2},
$$

and the " $a$ " that works for $r^{(2)}$ as in (3.1.1) is

$$
a^{(2)}=\left(y^{3} u+w_{1},-z^{3} v+w_{2}, 0\right) \text {. }
$$

Note that

$$
a^{(2)} \cdot h \equiv x^{2} u w_{1}+x^{2} u w_{2} \quad(\bmod g) .
$$

Now, according to (3.1.2) or more specifically the remark (2) of (3.2), the vanishing of the primary obstruction is equivalent to the existence of $\tilde{h}$ $=\left(\tilde{h}_{1}, \tilde{h}_{2}, \tilde{h}_{3}\right)$ subject to the two conditions

$$
\begin{array}{ll}
r^{(1)} \cdot \tilde{h} \equiv a^{(1)} \cdot h & (\bmod g), \\
r^{(2)} \cdot \tilde{h} \equiv a^{(2)} \cdot h & (\bmod g) .
\end{array}
$$

In view of (3)', (4)' above, these conditions are respectively

$$
\begin{array}{ll}
z^{2} \tilde{h}_{1}-y^{2} \tilde{h}_{2} \equiv 0 & (\bmod g), \\
-y^{5} \tilde{h}_{1}+z^{5} \tilde{h}_{2}-x^{2} \tilde{h}_{3} \equiv x^{2} u w_{1}+x^{2} v w_{2} & (\bmod g) .
\end{array}
$$

Finally, we observe that these conditions can always be satisfied by letting

$$
\tilde{h}_{1}=\tilde{h}_{2}=0, \quad \tilde{h}_{3}=-u w_{1}-v w_{2}-v w_{2} .
$$

Thus: There are no primary obstructions to the cone deformations of $C$.

To check that there are no higher obstructions, we proceed similarly, using (3.1.2) ff. to define successively the $h_{(n)}$ 's and $a_{(n)}$ 's. We summarize the situation in the table below; the cases $n=1,2$ already worked out are included, but now with the general subscript notation of (3.1.2). The computations are not difficult, especially since the relation $r^{(1)}$ ceases to be relevant for $n>1$, and the entire procedure trivializes after $n>3$. The notation $a_{(n)}^{(k)}$ means the coefficient of $\varepsilon^{n}$ in the extension of the relation $r^{(k)}(k=1,2)$ to a relation among the $\varphi_{(n) i}$, where

$$
\varphi_{(n)}=\varphi+h_{(1)} \varepsilon+\cdots+h_{(n)} \varepsilon^{n}
$$

(everything is, of course, a 3-tuple).

Note that there is no restriction on $u, v, w_{1}, w_{2}$ in $P_{2} \times P_{2} \times P_{5} \times P_{5}$ (where $P_{v}$ denotes the space of homogeneous polynomials of degree $\nu$ ). Choose polynomials $\left\{u_{\alpha}\right\},\left\{v_{\beta}\right\},\left\{w_{1 \gamma}\right\},\left\{w_{2 \delta}\right\}$ so that the elements $\left(x^{2} u_{\alpha}, x^{2} v_{\beta}, y^{2} w_{1 \gamma}+z^{2} w_{2 \delta}\right)$ are linearly independent modulo $g$ and the ideal generated by the first partial derivatives of the triple $\left(\varphi_{1}, \varphi_{2}, \varphi_{3}\right)$. Let $\left\{s_{\alpha}\right\},\left\{t_{\beta}\right\},\left\{q_{\gamma}\right\},\left\{q_{\delta}\right\}$ be the dual basis. 


\begin{tabular}{|c|c|c|}
\hline$n=0$ & $\begin{array}{l}a\left(\begin{array}{l}1 \\
0\end{array}\right)=r^{(1)}=\left(z^{2},-y^{2}, 0\right) \\
a\left(\begin{array}{l}2 \\
0\end{array}\right)=r^{(2)}=\left(-y^{5}, z^{5},-x^{2}\right)\end{array}$ & $h_{(0)}=\varphi=\left(x^{2} y^{2}, x^{2} z^{2}, z^{7}-y^{7}\right)$ \\
\hline$n=1$ & $\begin{array}{l}a(1)=(v,-u, 0) \\
a\left(\gamma_{1}^{2}\right)=\left(y^{3} u+w_{1},-z^{3} v+w_{2}, 0\right)\end{array}$ & $h_{1}=\left(x^{2} u, x^{2} v, y^{2} w_{1}+z^{2} w_{2}\right)$ \\
\hline$n=2$ & $\begin{array}{l}a\left(\begin{array}{l}1 \\
2\end{array}\right)=(0,0,0) \\
a(2)=\left(-y u^{2}, z v^{2}, 0\right)\end{array}$ & $h_{(2)}=\left(0,0, u w_{1}+v w_{2}\right)$ \\
\hline$n=3$ & $\begin{array}{l}a\left(\begin{array}{l}1 \\
3\end{array}\right)=(0,0,0) \\
a\left(\begin{array}{l}2 \\
(\end{array}\right)=(0,0,0)\end{array}$ & $h_{(3)}=\left(0,0,-y u^{3}+z v^{3}\right)$ \\
\hline & : & \\
\hline
\end{tabular}

everything 0 thereafter.

TABLE (3.3.1)

Then

(3.3.2) The formal cone-moduli space of $C$ is Spec $(R)$, where

$$
\left.R=k\left[\left[s_{\alpha}\right\},\left\{t_{\beta}\right\},\left\{p_{\gamma}\right\},\left\{q_{\delta}\right\}\right]\right]
$$

the versal family of cones over $R$ is (according to Table (3.3.1) above) given by the ideal generated by

$$
\varphi_{1}+x^{2} u, \quad \varphi_{2}+x^{2} v, \quad \varphi_{3}+y^{2} w_{1}+z^{2} w_{2}+u w_{1}+v w_{2}-y u^{3}+z v^{3}
$$

where $u=\Sigma_{\alpha} s_{\alpha} u_{\alpha}, v=\Sigma_{\beta} t_{\beta} v_{\beta}, w_{1}=\Sigma_{\gamma} p_{\gamma} w_{1 \gamma}, w_{2}=\sum_{\delta} q_{\delta} w_{2 \delta}$.

(3.4) We now consider the normally flat deformations of $X$. Since $X \hookrightarrow A^{3}$ is the complete intersection $f_{1}=f_{2}=0$, a deformation of $X$ is given by arbitrary deformations of $f_{1}, f_{2}$. In particular, over $k[\varepsilon] / \varepsilon^{2}$ the deformations are given by

$$
f_{i}^{\prime}=f_{i}+\varepsilon g_{i} \quad(i=1,2),
$$

$g_{1}, g_{2}$ being arbitrary. We wish to determine when this family is normally flat along the 0 -section $x=y=z=0$. Let $J^{\prime}=\left(f_{1}^{\prime}, f_{2}^{\prime}\right) k[\varepsilon, x, y, z] / \varepsilon^{2}$. Since $f_{1}, f_{2}$ and $f_{3}=z^{2} f_{1}-y^{2} f_{2}$ are an $M$-standard base of $J($ see (3.0)), by Theorem (2.13) the condition is that there exist $\tilde{f}_{i} \in J^{\prime}(i=1,2,3)$ such that $\tilde{f}_{i}(\bmod \varepsilon)$ $=f_{i}$, and $\nu_{M}\left(\tilde{f}_{i}\right)=\nu_{M}\left(f_{i}\right)$.

Suppose this condition is satisfied, i.e. suppose in $J^{\prime}$ we have $\tilde{f}_{i}=f_{i}+\varepsilon \tilde{g}_{i}$, 
with $\tilde{g}_{1}, \tilde{g}_{2} \in M^{4}, \tilde{g}_{2} \in M^{7}$. Then by flatness, we must have $g_{i}-\tilde{g}_{i} \in J(i$ $=1,2)$, and $z^{2} g_{1}-y^{2} g_{2}-\tilde{g} 3 \in J$. Thus the normal flatness implies

$$
\begin{cases}g_{i} \in M^{4}+J, \quad i=1,2 & \text { (i.e. } \left.g_{i} \equiv \tilde{g}_{i}(\bmod J), \tilde{g}_{i} \in M^{4}\right), \\ z^{2} g_{1}-y^{2} g_{2} \in M^{7}+J & \text { (i.e. } \left.z^{2} g_{1}-y^{2} g_{2} \equiv \tilde{g}_{3}(\bmod J), \tilde{g}_{3} \in M^{7}\right) .\end{cases}
$$

Conversely, if these conditions hold for $g_{1}, g_{2}$ the deformation they define is normally flat among the 0 -section. In fact, given (3.4.1) suppose that

$$
g_{i}=\tilde{g}_{i}+a_{i 1} f_{1}+a_{i 2} f_{2}, \quad \tilde{g}_{i} \in M^{4}, i=1,2,
$$

and

$$
z^{2} g_{1}-y^{2} g^{2}=g_{3}+b_{1} f_{1}+b_{2} f_{2}, \quad \tilde{g}_{3} \in M^{7} .
$$

Then, letting

$$
\begin{aligned}
& \tilde{f}_{i}=f_{i}^{\prime}-\varepsilon\left(a_{i 1} f_{1}+a_{i 2} f_{2}\right), \quad i=1,2, \\
& \tilde{f}_{3}=z^{2} f_{1}^{\prime}-y^{2} f_{2}^{\prime}-\varepsilon\left(b_{1} f_{1}^{\prime}+b_{2} f_{2}^{\prime}\right),
\end{aligned}
$$

we see that $\tilde{f}_{i}=f_{i}+\varepsilon \tilde{g}_{i}$ and is in $J^{\prime}$ for $i=1,2,3$, so the conditions of Theorem (2.13) are satisfied. Moreover, since the $f_{i}$ are then an $(x, y, z)$ standard base of $J^{\prime}$, the cone-deformation associated to $J^{\prime}$ by the morphism $u$, i.e. the normal cone to the 0 -section viewed as a deformation of $C$, is given by the $\operatorname{In}_{(x, y, z)}\left(\tilde{f}_{i}\right)=\varphi_{i}+\varepsilon h_{i}$, where $h_{i}=\operatorname{In}_{(x, y, z)}\left(g_{i}\right), i=1,2,3$.

We now ask: what is the image of the map of tangent spaces corresponding to $\mathbf{u}$ ? In view of the above, this amounts to the following:Given homogeneous polynomials $\left(h_{1}, h_{2}, h_{3}\right)$ of degrees $(4,4,7)$ satisfying $(3.1 .1)$, does there exist $g_{1}, g_{2}$ so that (with the notation of (3.4)) the ideal $J^{\prime}$ contains $f_{i}+\varepsilon \tilde{g}_{i}$ with $h_{i}=\operatorname{In}_{(x, y, z)}\left(\tilde{g}_{i}\right), i=1,2,3$ ? To answer this question, we may clearly start by assuming that already $g_{1}=\tilde{g}_{1}, g_{2}=\tilde{g}_{2}$ are in $M^{4}$ with $\operatorname{In}_{(x, y, z)}\left(\tilde{g}_{i}\right)=h_{i}$ for $i=1,2$. Then by (3.4.1), the condition that $\left(h_{1}, h_{2}, h_{3}\right)$ be in the image of $\mathbf{u}$ is

$$
z^{2} g_{1}-y^{2} g_{2}-h_{3} \in J+M^{8}
$$

Now write $g_{1}=h_{1}+a, g_{2}=h_{2}+b$ with $a, b \in M^{5}$. We know from (3.3) that $h_{1}=x^{2} u, h_{2}=x^{2} v$ for some $u, v$ of degree 2 , so that $z^{2} h_{1}-y^{2} h_{2}$ $=-v \varphi_{1}+u \varphi_{2}$. Thus the expression on the left in (*) is

$$
\begin{aligned}
z^{2} h_{1}+z^{2} a-y^{2} h_{2}-y^{2} b-h_{3} & =-v \varphi_{1}+u \varphi_{2}+z^{2} a-y^{2} b-h_{3} \\
& =-v\left(f_{1}-z^{5}-x^{6}\right)+u\left(f_{2}-y^{5}\right)+z^{2} a-y^{2} b .
\end{aligned}
$$

Hence (*) is equivalent to 


$$
v z^{5}-u y^{5}+z^{2} a-y^{2} b-h_{3} \in J+M^{8} \text {. }
$$

Now, since $z^{2} a, y^{2} b$ are in $M^{7}$, the validity of the above is not affected if we replace $a, b$ by their initial forms. Assuming this done, the condition is

$$
u z^{5}-u y^{5}+z^{2} a+y^{2} b-h_{3} \in \mathcal{g},
$$

where the expression on the left is homogeneous of degree 7. Now recall that $h_{3}$ is necessarily of the form $y^{2} w_{1}+z^{2} w_{2}$, where $w_{1}, w_{2}$ are arbitrary forms of degree 5 , and in particular are independent of $u, v$. Thus if we identify a cone deformation over $k[\varepsilon] / \varepsilon^{2}$ with the data $(u, v, w)$ we see from $(* *)$ that it is in the image of $\mathbf{u}\left({ }^{10}\right)$ if and only if there exist forms $a$ and $b$ of degree 7 so that

$$
y^{2}\left(-u y^{3}-b-w_{1}\right)+z^{2}\left(v z^{3}+a-w_{2}\right) \in g .
$$

In particular, if we let $a=w_{2}-v z^{3}, b=-w_{1}-u y^{3}$, this condition is trivially satisfied. Thus

(3.4.2) The deformation of $X$ over $k[\varepsilon] / \varepsilon^{2}$ given by

$$
\tilde{f}_{1}=f_{1}+\varepsilon\left(x^{2} u+w_{2}-v z^{3}\right), \quad \tilde{f}_{2}=f_{2}+\varepsilon\left(x^{2} v-w_{1}-u y^{3}\right)
$$

is normally flat along the 0-section, and the corresponding cone deformation is $\left(u, v, w_{1}, w_{2}\right)$ (thus the morphism $\mathbf{u}$ is surjective on tangent spaces). In fact, we note that $\varphi_{3}+y^{2} w_{1}+z^{2} w_{2}$ is the initial form of the element

$$
\tilde{f}_{3}=\left(z^{2}+v\right) \tilde{f}_{1}-\left(y^{2}+u\right) \tilde{f}_{2} .
$$

This situation is perhaps better expressed if we view $u, v, w_{1}, w_{2}$ as depending on the parameters $s_{\alpha}, t_{\beta}, p_{\gamma}, q_{\delta}$ as in (3.3.2). We may then dispense with $\varepsilon$, and state simply that up to the first order in these parameters the versal cone deformation is the normal cone to the 0 -section of the family

$$
\tilde{f}_{1}=f_{1}+x^{2} u+w_{2}-v z^{3}, \tilde{f}_{2}=f_{2}+x^{2} v-w_{1}-u y^{3} \text {. }
$$

Let us extend this family by adding some second-order terms: we now let $\tilde{f}_{1}, \tilde{f}_{2}$ be defined by

$$
\begin{aligned}
& \tilde{f}_{1}=f_{1}+x^{2} u+w_{2}-v z^{3}+v^{2} z, \\
& \tilde{f}_{2}=f_{2}+x^{2} v-w_{1}-u y^{3}+u^{2} y .
\end{aligned}
$$

Then we check that the element $\tilde{f}_{3}=\left(z^{2}+v\right) \tilde{f}_{1}-\left(y^{2}+u\right) \tilde{f}_{2}$ viewed, say in $k\left[\left[s_{\alpha}, t_{\beta}, p_{\gamma}, q_{\delta}\right]\right][x, y, z]$ has as its initial form

(10) The symbols $\mathbf{u}$ and $u$ should not be confused. The former is a morphism of functors, the latter is a form of degree 2. 


$$
\varphi_{3}+y^{2} w_{1}+z^{2} w_{2}+u w_{1}+u w_{2}-y u^{3}+z v^{3} .
$$

Note also that the initial forms of $\tilde{f}_{1}$ and $\tilde{f}_{2}$ are $\varphi_{1}+x^{2} u$ and $\varphi_{1}+x^{2} v$. Thus we deduce from (3.3.2) that

(3.4.4) The normal cone to the 0-section of the deformation of $X$ given over $k\left[\left[s_{\alpha}, t_{\beta}, p_{\gamma}, q_{\delta}\right]\right]$ by $\tilde{f}_{1}, \tilde{f}_{2}$ in (3.4.3) above is the versal cone deformation of $C$.

Now suppose we denote by $\omega_{1}$ and $\omega_{2}$ two sets of parameters for the general form of degree 5 in $x, y, z$, i.e.

$$
F_{i}=\sum_{\tau} \omega_{i \tau} M_{\tau}, \quad i=1 \text { or } 2,
$$

where $\left\{M_{\tau}\right\}_{\tau}$ runs over the district monomials. Then we observe that the deformation (3.4.3) can be rewritten as the family

$$
\tilde{f}_{1}=f_{1}+x^{2} u+F_{1}, \quad \tilde{f}_{2}=f_{2}+x^{2} v+F_{2}
$$

over the parameter space $T$ with the coordinate functions $s_{\alpha}, t_{\beta}, \omega_{1 \tau}, \omega_{2 \tau}$; we simply collect all the degree 5 terms in (3.4.3) together. Now let us preserve the notation $s_{\alpha}, t_{\beta}, p_{\gamma}, q_{\delta}$ for the coordinate functions on the parameter space $U$ of the versal family $\mathcal{C}$ of cones. The identification of $\mathcal{C}$ with the normal cone to the 0 -section of (3.4.5) is accomplished via the isomorphism $T \stackrel{\approx}{\longrightarrow} U$ of the parameter spaces given by

$$
\begin{aligned}
& s_{\alpha} \mapsto s_{\alpha}, \quad t_{\beta} \mapsto t_{\beta}, \\
& p_{\gamma} \mapsto \sigma_{\gamma}^{\prime}\left(\omega_{2 \tau, s_{\alpha}}\right), \quad q_{\delta} \mapsto \sigma_{\delta}^{\prime \prime}\left(\omega_{1 \tau, t_{\beta}}\right),
\end{aligned}
$$

where $\sigma_{\gamma}^{\prime}\left(\omega_{2 \tau, s_{\alpha}}\right)$ and $\sigma_{\delta}^{\prime \prime}\left(\omega_{1 \tau, t_{\beta}}\right)$ are the coefficients of $w_{1 \gamma}$ and $w_{2 \delta}$, respectively, in the forms

$$
-F_{2}-u y^{3}+u^{2} y \text { and }-F_{1}+v z^{3}-v^{2} z .
$$

This is of course essentially a tautology since we are viewing $F_{i}$ as the total part of degree 5 in $\tilde{f}_{i}(i=1,2)$; we can then use (3.4.3) to express $w_{i}$ in terms of $u$, $v$ and the $F_{i}$.

Now let $G_{1}$ and $G_{2}$ denote arbitrary polynomials in $x, y, z$ with $\nu_{(x, y, z)}\left(G_{i}\right)$ $>5, i=1,2$. If we add $G_{i}$ on to $\tilde{f}_{i}$ in (3.4.5) we still get a normally flat deformation; for the effect of the $G_{i}$ on $\tilde{f}_{3}$, defined say as above to be $\left(z^{2}+v\right) \tilde{f}_{1}-\left(y^{2}+u\right) \tilde{f}_{2}$, is to add terms of order at least 8 . Thus the $G_{i}$ do not effect the initial forms of $\tilde{f}_{1}, \tilde{f}_{2}, \tilde{f}_{3}$ at all. It follows that if we let $N$ denote the formal space of choices $\left(u, v, F_{1}, F_{2}, G_{1}, G_{2}\right)$, then the normal cone to the 0 section of our new big family $\mathfrak{X}$ over $N$ defined by

$$
\tilde{f}_{1}=f_{1}+x^{2} u+F_{1}+G_{1}, \quad \tilde{f}_{2}=f_{2}+x^{2} v+F_{2}+G_{2}
$$


is just $N \times_{T} \mathrm{e}$. Here $N \rightarrow T$ is the projection onto $u, v, F_{1}, F_{2}$ and $\mathrm{C}$ is the normal cone to the 0 -section of the family (3.4.5) over $T$ (or the versal family of cones via the identification induced by the isomorphism $T \rightarrow U$ described above).

More precisely, let

$$
N=\operatorname{Spec} k\left[\left[s, t, \omega_{1}, \omega_{2}, \eta\right]\right]
$$

where $s, t, \omega_{1}, \omega_{2}$ are the coordinate functions on $T$, and $\eta$ represents a new set of functions corresponding to a parametrization of the space of pairs of polynomials $G_{1}, G_{2}$ (modulo equivalence of deformations, which insures finiteness). Thus we may view the family $\mathfrak{X}$ of (3.4.6) as defined over $N$. Let the morphism $\rho: N \rightarrow U$ denote the composition of the projection $N \rightarrow T$ and the isomorphism $T \stackrel{\longrightarrow}{\longrightarrow}$ described earlier. Then

(3.4.7) If $\mathcal{C}$ denotes the versal family of cones, the pull-back of $\mathcal{C}$ to $N$ via $\rho$ is the normal cone to the 0-section of (3.4.6) (i.e. $N \times_{U}$ C is $\mathbf{u}$ applied to the family (3.4.6) together with its normally-flat 0-section).

(3.5) Now we want to show that the family $\mathfrak{X}$ given by (3.4.6) over $N$ is, once we suitably interpret the notation, the versal normally flat family. It will then follow from (3.4.7) that the morphism of functors $u$ is represented by $\rho$, so that $\mathbf{u}$ is smooth in this case. In order to proceed, we will have to assume a result will be proved in Chapter 4, (4.49): The tangent space to the normally flat deformations of $X$ at $P$ is a direct sum $T_{X, P} \oplus 干_{0}^{1}$, where:

(1) $T_{X, P}$ is the "strict tangent space to $X$ at $P$ ", i.e. the largest linear subspace (of the Zariski tangent space) which splits off the tangent cone $C_{X, P}$.

(2) $T_{0}^{1}$ is the filtered piece of degree 0 of the tangent space $T^{1}$ to the ordinary deformation theory of $X$ at $P$, with respect to a certain filtration which we now describe: Let $R, M$ and $\theta, \mathrm{m}$ be the local rings at $P$ of the ambient space and of $X$ respectively. Let $\theta=R / J$. Then we know $T^{l}$ is a quotient of $\operatorname{Hom}_{\theta}\left(J / J^{2}, \theta\right)$ by the canonical image of $\operatorname{Der}_{k}(\theta, \theta)$; If $\left\{f_{i}\right\}$ is an ideal base of $J$ then a first-order deformation $f_{i}+\varepsilon g_{i}$ corresponds to the homomorphism which sends the class of $f_{i}\left(\bmod J^{2}\right)$ to $g_{i}$. Now if $\left\{f_{i}\right\}$ is an $M$-standard base of $J$, with $\nu_{i}=\nu_{M}\left(f_{i}\right)$, we will say a homomorphism $h$ has degree $d$ if $h$ (class of $\left.f_{i}\right)$ is in $\mathbf{m}^{v_{i}+d}$ for all $i$. This defines a filtration on $\operatorname{Hom}_{\mathcal{O}}\left(J / J^{2}, \mathcal{O}\right)$, and hence on $T^{l}$.

To see the plausibility of this definition, note that $T_{0}^{1}$ as defined above should essentially be viewed as those deformations which (modulo coordinate change) are given by $\left\{f_{i}+\varepsilon g_{i}\right\}$ with $\nu_{M}\left(g_{i}\right) \geqslant \nu_{i}$; this coincides with the space of deformations which are normally flat along the 0 -section.

Now to return to our situation, one first checks that in our case $\Psi_{X, P}=(0)$ (in other words all of the variables are "necessary" to give the equations of the tangent cone $C$ ). Thus the tangent space to the normally flat deformations is 
just $T_{0}^{1}$. Now we have seen that the only choices of $g_{1}, g_{2}$ for which $f_{1}+\varepsilon g_{1}, f_{2}+\varepsilon g_{2}$ is normally flat along the 0 -section are $g_{1}=x^{2} u+$ higherorder terms, $g_{2}=x^{2} v+$ higher-order terms, where $u$ and $v$ are forms of degree 2 ; in fact, it turned out (3.4) that this property of $g_{1}, g_{2}$ is the same as saying that $z^{2} g_{2}-y^{2} g_{1}$ is in $M^{7}+J$. On the other hand, given any homomorphism $h: J / J^{2} \rightarrow \mathcal{O}$, with $h\left(f_{1}\right)=g_{1}, h\left(f_{2}\right)=g_{2}$, then $h\left(f_{3}\right)$ must be $z^{2} g_{2}$ $-y^{2} g_{1}(\bmod J)$. Hence $T_{0}^{1}$ is a quotient of the space of $h \in \operatorname{Hom}\left(J / J^{2}, \theta\right)$ with $h\left(f_{1}\right)=g_{1}, h\left(f_{2}\right)=g_{2}$ where $g_{1}, g_{2}$ are as above.

Now if we recall the definition of $N$ and the family $\mathfrak{X}$ of (3.4.6), we see that the conclusion of the previous paragraph is the same as the statement: $T_{0}^{1}$ is a quotient of the Zariski tangent space $V$ of $N$; the projection $V \rightarrow T_{0}^{1}$ is obtained by viewing the restriction of $\mathfrak{X}$ to a tangent vector of $N$ as a family over $k[\varepsilon] / \varepsilon^{2}$ which is normally flat along the 0 -section. Thus if we restrict ourselves to a suitable subspace of the space of coordinate functions $s, t, \omega_{1}$, $\omega_{2}, \eta$ of $N$, and correspondingly restrict the parameters of variation of (3.4.6), we find that (preserving the notation) we still have a normally flat family $\mathfrak{X}$ over a smooth space $N$, but now it is the versal one up to first order. It follows that it must be a versal one (of all orders); for this one can use the same abstract argument used by Schlessinger [4] to construct versal families; the point here is that $N$ is smooth, so the family $\mathfrak{X}$ can be viewed as the limit of successive "maximal" infinitesimal extensions of the versal first order family.

A word must be said about the meaningfulness of (3.4.7) in view of this "smaller" sense of $N$ that we adopted in the paragraph above. One first observes that the original morphism $\rho: N \rightarrow U$ must factor through $T_{0}^{1}$ (on the level of tangent spaces) by functoriality. It is then not hard to interpret $\rho$ as being well defined in the new sense of $N$, in such a way that (3.4.7) is still valid. We leave the details here to the reader.

\section{Tangent spaces.}

(4.0) In this section we study the tangent spaces to the functors $\tau_{j}$ of (infinitesimal) normally flat deformations of order $j$ of an isolated singularity $(X, x)$. We denote by $F$ the "ordinary" deformation functor of $X$ over $k$-we assume $x$ is a $k$-rational point of $X$ as usual. In particular, the tangent space $t_{F}$ of $F$ is $F\left(k[\varepsilon] / \varepsilon^{2}\right)$, the set of isomorphism classes of (flat) deformations of $X$ over the dual numbers. We will obtain a decomposition of the tangent space $t_{\varkappa_{j}}$ to $\varkappa_{j}$ into the sums of a certain subspace of $t_{F}$ and a space of certain sections of the trivial deformation; the precise definitions depend on the results of $\$ 2$. This decomposition is useful (especially in the strict complete intersection case and in particular the hypersurface case) to interpret the singularities of "higher discriminant loci" in the formal moduli.

To begin, we recall briefly the vector space structure on $t_{F}$ (for details the reader is referred to [4],): Let $D=\operatorname{Spec}\left(k[\varepsilon] / \varepsilon^{2}\right)$. We have $k$-morphisms 


$$
\alpha: D \rightarrow D \oplus_{k} D \quad\left(=\operatorname{Spec}\left(k[\varepsilon] / \varepsilon^{2} \times_{k} k[\varepsilon] / \varepsilon^{2}\right)\right)
$$

and

$$
\mu_{b}: D \rightarrow D \quad(b \in k)
$$

$\alpha$ is given (on rings) by $(\varepsilon, 0) \mapsto \varepsilon$ and $(0, \varepsilon) \mapsto \varepsilon$, and $\mu_{b}$ by $\varepsilon \mapsto b \varepsilon$. These morphisms induce the addition and scalar multiplication on $t_{F}$ as follows: If $Z_{1}, Z_{2} \in t_{F}$, we define $Z_{1}+Z_{2}$ to be $\alpha^{*}\left(Z_{1} \oplus_{X} Z_{2}\right)$ (note $Z_{1} \oplus_{X} Z_{2}$ lies naturally over $D \oplus_{k} D$, since $X$ is the fibre of $Z_{i}$ over Spec $(k)$, the closed point); similarly for $Z \in t_{F}, b F$ is $\mu_{b}^{*}(Z)$.

Concretely, suppose $X=\operatorname{Spec}\left(k\left[x_{1}, \ldots, x_{n}\right] / J\right)$, and say $J=\left(f_{1}, \ldots, f_{s}\right)$ is any ideal base. Then we may assume that for $i=1,2$,

$$
Z_{i}=\operatorname{Spec} k[\varepsilon, x] /\left(f_{1}+\varepsilon g_{1}^{(i)}, \ldots, f_{s}+\varepsilon g_{s}^{(i)}\right)
$$

with the $g_{j}^{(i)} \in k[x]$. One checks easily that then

$$
\text { (4.0.1) } Z_{1}+Z_{2}=\operatorname{Spec}\left(k[\varepsilon, x] /\left(f_{1}+\varepsilon\left(g_{1}^{(1)}+g_{1}^{(2)}\right), \ldots, f_{s}+\varepsilon\left(g_{s}^{(1)}+g_{s}^{(2)}\right)\right)\right) \text {. }
$$

Similarly, if $Z$ is given by $f_{j}+\varepsilon g_{j}, j=1, \ldots, s$, then for $b \in k, b Z$ is given by $f_{j}+\varepsilon b g_{j}$.

Now suppose $Z_{1}$ and $Z_{2}$ are given in $t_{F}$ together with sections $\sigma_{i}: D \rightarrow Z_{i}$, which pass through the singular point $x$ of the closed fibre $X$. Then we define $\left(\sigma_{1}+\sigma_{2}\right): D \rightarrow Z_{1}+Z_{2}$ to be $\alpha^{*}\left(\sigma_{1} \oplus \sigma_{2}\right)$, where $\sigma_{1} \oplus \sigma_{2}: D \oplus_{k} D \rightarrow Z_{1}$ $\oplus_{X} Z_{2}$ is the natural morphism determined by $\sigma_{i}$ on the $i$ th summand-thus:

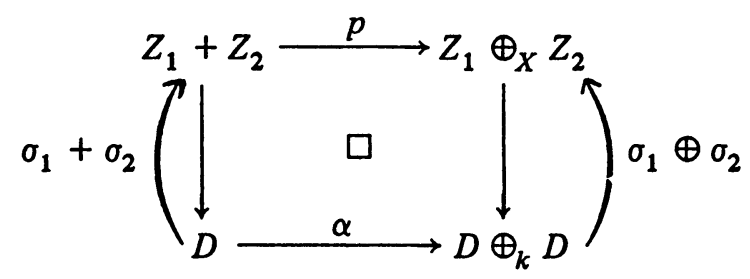

Similarly we define $b \sigma$ to be $\mu_{b}^{*}(\sigma)$ for $b$ in $k$, and $Z \in t_{F}$ with section $\sigma$.

Explicitly, given $Z_{1}, Z_{2}$ as above, let $I$ be the ideal in $\theta_{Z_{1} \oplus_{X}} Z_{2}$ which defines $\sigma_{1} \oplus \sigma_{2}$. Then the ideal of $\sigma_{1}+\sigma_{2}$ is obtained by lifting $I$ to $Z_{1}+Z_{2}$ via $p$ (see the diagram above). Now if $B_{i}$ is the affine ring of $Z_{i}$, and if $I_{i}$ is the ideal of $\sigma_{i}$ in $B_{i}$, then $I=I_{1} \times_{B} I_{2} \subset \theta_{Z_{1} \oplus_{X} Z_{2}}$ (say $X=\operatorname{Spec}(B)=\operatorname{Spec}\left(B_{i} / \varepsilon B_{i}\right)$ ). Assume that $Z_{i} \hookrightarrow A_{D}^{n}=\operatorname{Spec}\left(k\left[\varepsilon, x_{1}, \ldots, x_{n}\right]\right)$, and that $x$ is the origin of the closed fibre. Then, in the ambient space,

$$
I_{i}=\left\{x_{1}-a_{1}^{(i)} \varepsilon, \ldots, x_{n}-a_{n}^{(i)} \varepsilon\right\} k\left[\varepsilon, x_{1}, \ldots, x_{n}\right],
$$

for some $a_{j}^{(i)} \in k$, so that 


$$
I_{1} \times I_{2}=\left\{\left(x_{j}-a_{j}^{(1)} \varepsilon^{(1)}, x_{j}-a_{j}^{(2)} \varepsilon^{(2)}\right)\right\}_{j=1}^{n} k\left[\varepsilon^{(1)}, \varepsilon^{(2)}, x_{1}, \ldots, x_{n}\right] .
$$

(Here we view

$$
Z_{1} \oplus_{X} Z_{2} \hookrightarrow \mathbf{A}_{D}^{n} \oplus_{A_{k}^{n}} \mathbf{A}_{D}^{n}=\operatorname{Spec} k\left[\varepsilon^{(1)}, \varepsilon^{(2)}, x_{1}, \ldots, x_{n}\right]
$$

$\varepsilon^{(1)}, \varepsilon^{(2)}$ correspond to $(\varepsilon, 0)$ and $(0, \varepsilon)$.) Consequently, the ideal of $\sigma_{1}+\sigma_{2}$ is (ambiently):

$$
\left\{x_{j}-\left(a_{j}^{(1)}+a_{j}^{(2)}\right) \varepsilon\right\}_{j=1}^{n} k\left[\varepsilon, x_{1}, \ldots, x_{n}\right]
$$

(4.1) Now let $\pi: \mathfrak{X} \rightarrow S$ be the versal formal deformation of $X$ in the sense of Schlessinger [4]. (We will view $S$ as the spectrum of a complete local ring $R$, rather than a formal scheme.) Let $N_{j}$ denote the intersection of the components through $x$ (the distinguished singular point of $X$ now viewed as the closed fibre of $\pi$ ) of the flat strata of $P_{\mathfrak{X} / S}^{n}, n \leqslant j$. We have seen in $\S 1$, (1.2.4), that $N_{j}$ is the "prorepresentable hull" of the functor $\Re_{j}$ for $(X, x)$, and that the versal formal normally flat deformation of order $j$ is

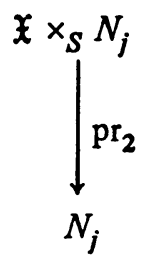

(The versal section is $\left(i, 1_{N_{j}}\right), i$ being the inclusion of the closed subscheme $N_{j} \hookrightarrow \mathfrak{X}$.) In particular the tangent space to the functor $\Re_{j}$, i.e. $\Re_{j}\left(k[\varepsilon] / \varepsilon^{2}\right)$ is $T_{N_{j, x}}$, the tangent space to the $k$-scheme $N_{j}$ at the closed point $x$. Recall $(\$ 1,1.1 .1)$ that for an artin local $k$-algebra $A$ with residue field $k$,

$$
\begin{aligned}
& \mathscr{\vartheta}_{j}(A)={ }_{\text {def }}\{(Z, \sigma) \mid Z \in F(A), \sigma: \text { Spec }(A) \\
&\left.\rightarrow Z \text { a section such that } \operatorname{Gr}_{\sigma}(Z) \text { is flat } / A \text { through degree } j\right\} .
\end{aligned}
$$

We note that the forgetful morphism of functors $\varkappa_{j} \rightarrow F$ which forgets the section $\sigma$ is represented by $\pi \circ i: N_{j} \rightarrow S$. Let $S_{j}$ denote the (scheme-theoretic) image of this map $\left({ }^{11}\right)$ :

(11)N.B.: The functor represented by $S_{j}$ contains, but is not necessarily a prorepresentable hull of the image of the morphism of functors. The point is that $N_{j}$ is finite, but not in general etale-or even flat-over $S_{j}$; for example in the case when $(X, x)$ is a strict complete intersection $N_{j} \rightarrow S_{j}$ is the normalization (char 0 ). 


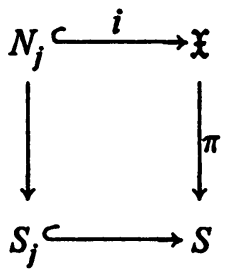

Thus we have a diagram of tangent spaces

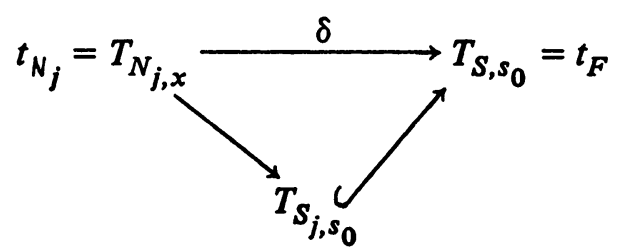

where $s_{0}$ denotes the closed point of $S$, and $\delta$ is the map on tangent spaces induced by $\left.\pi\right|_{N_{j}}$. Let $K_{j}$ denote the kernel of $\delta$.

(4.2) Our first step is to study $K_{j}$. It is clear from the definition that $K_{j}$ is the space of $j$-sections of the trivial deformation (over $k[\varepsilon] / \varepsilon^{2}$ ), i.e. of $X \times_{k} D$, which pass through $x$, the origin of the closed fibre. Now if $X \hookrightarrow A_{k}^{n}$, so that $X \times_{k} D \hookrightarrow \operatorname{Spec}\left(k\left[\varepsilon, x_{1}, \ldots, x_{n}\right]\right)=\mathbf{A}_{D}^{n}$, any section $\sigma$ through the origin corresponds uniquely to a tangent vector $a: D \rightarrow X$ at $x$. The image of $\sigma$ is then of the form $z_{1}=\cdots=z_{n}=0$, where $z_{i}=x_{i}-a_{i} \varepsilon$; we think of $a$ $=\left(a_{1}, \ldots, a_{n}\right) \in k^{n}=T_{X, x}$.

Let $J$ denote the ideal of $X$ in $\mathbf{A}_{k}^{n}$, and choose a standard base $f_{1}, \ldots, f_{s}$ of $J$ with respect to $M$, the maximal ideal of the origin ((2.2)). Then in terms of the new coordinate system $z_{i}$ in $\mathbf{A}_{D}^{n}$, the trivial family is defined by the ideal

$$
J^{\prime}=\left(f_{1}(z+a \varepsilon), \ldots, f_{s}(z+a \varepsilon)\right) k[\varepsilon, z]
$$

where $z=\left(z_{1}, \ldots, z_{n}\right), a=\left(a_{1}, \ldots, a_{n}\right)$. Let $\nu_{i}=\nu_{M}\left(f_{i}\right)$.

(4.2.1) Proposition. The section $\sigma$ of the trivial family given by $x_{i} \mapsto a_{i} \varepsilon$ is in $K_{j}$ if and only if

$$
a \cdot \nabla f_{i} \in M^{v_{i}}+J \text { for } i \text { such that } \nu_{i} \leqslant j
$$

and

$$
a \cdot \nabla f_{i} \in M^{j+1}+J \text { for the remaining } i .
$$

(Here $\nabla f_{i}=\left(\partial f_{i} / \partial x_{1}, \ldots, \partial f_{i} / \partial x_{n}\right)$ is the "gradient" of $f_{i}$ )

Proof. We note that the ideal $I$ of $\sigma$ is generated by $z_{1}, \ldots, z_{n}$ where $z_{i}=x_{i}-a_{i} \varepsilon$. Hence by (2.14), $\sigma \in K_{j}$-i.e., $\sigma$ is normally flat of order $j$-if and only if there exist $g_{i} \in J^{\prime}, i=1, \ldots, s$, with $g_{i}(\bmod \varepsilon)=f_{i}$ and such that: 


$$
\begin{array}{ll}
\nu_{(z)}\left(g_{i}\right) \geqslant \nu_{i} & \text { for those } i \text { with } \nu_{i}<j, \\
\nu_{(z)}\left(g_{i}\right)>j & \text { for the remaining } i .
\end{array}
$$

Now such a $g_{i}$ is of the form $f_{i}(z)+\varepsilon h_{i}(z)$ where $\nu_{M}\left(h_{i}\right) \geqslant \nu_{i}$ (resp. $\nu_{M}\left(h_{i}\right)$ $>j)$. On the other hand, by the description of $J^{\prime}$ above, $f_{i}(z+a \varepsilon)=f_{i}(z)$ $+\varepsilon a \cdot \nabla f_{i}(z)$ is also in $J^{\prime}$, so that $\varepsilon\left(h_{i}-a \cdot \nabla f_{i}\right) \in J^{\prime}$, i.e. $h_{i}-a \cdot \nabla f_{i} \in J$. Thus the existence of the $g_{i}$ is equivalent to the existence of $h_{i}$ with $\nu_{M}\left(h_{i}\right) \geqslant \nu_{i}$ $(\operatorname{resp}>j)$ and $a \cdot \nabla f_{i} \equiv h_{i}(\bmod J)$; this is evidently the same as the assertion of (4.2.1). Q.E.D.

(4.2.2) REMARK. Suppose $X$ is a hypersurface, so that $J=(f)$, with, say $\nu_{M}(f)=\nu$. Then (4.2.1) may be stated more simply: $\sigma$ is normally flat of order $j(\leqslant \nu-1)$ if and only if

$$
\nu_{M}(a \cdot \nabla f)>j
$$

(with notations as above). In fact, by (2.6.1), the condition is that $J^{\prime}$ $=(f(z)+\varepsilon h(z))$ with $\nu_{(z)}(h)>j$ if $j \leqslant \nu-1$ (normal flatness of order $j$ $\geqslant \nu-1$ is equivalent to normal flatness of all orders, so the restriction on $j$ is only apparent). Now just as in (4.2.1), if $f+\varepsilon h \in J^{\prime}$, then $a \cdot \nabla f-h \in J$, i.e. $a \cdot \nabla f \in M^{j+1}+J$. However, since $J \subset M^{\nu}$, and $j \leqslant \nu-1$, the condition is simply (*).

(4.2.3) ExAmple. Let $X$ be the cusp $f(x, y)=y^{2}+x^{3}=0$. Then $\nabla f$ $=\left(3 x^{2}, y\right)$, and the vectors $a=\left(a_{1}, a_{2}\right)$ which satisfy $(*)$ above for $j=1$ (normal flatness of all orders) are the multiples of $(1,0)$.

Returning to the general situation, it will be clarifying to interpret the conditions of (4.2.1) as conditions on initial forms. For this, let $\varphi_{i}=\operatorname{In}_{M}\left(f_{i}\right)$, $\nu_{i}=\nu_{M}\left(f_{i}\right)$, and everything is as in (4.2.1). In addition we will denote $g=\mathrm{Gr}_{M}(J, R)$. Now given $a \in k^{n}=T_{X, x}$, notice that the condition $a \cdot \nabla f_{i}$ $\in M^{\nu_{i}}+J$ is equivalent to the condition $a \cdot \nabla \varphi_{i} \in \mathcal{g}$, since the only possible terms of degree $\left\langle\nu_{i}\right.$ in $a \cdot \nabla f_{i}$ are those of degree $\nu_{i}-1$ coming from $a \cdot \nabla \varphi_{i}\left(f_{i}=\varphi_{i}+\right.$ higher order terms $)$. Similarly, if $\nu_{i}>j$, the condition $a \cdot \nabla f_{i} \in M^{j+1}+J$ is equivalent to $a \cdot \nabla \varphi_{i} \in \Re^{j+1}+g$ where $\Re$ denotes the ideal of the vertex in $\mathrm{Gr}_{M}(R)$. Thus (4.2.1) becomes:

(4.2.4) If we identify sections $\sigma$ of the trivial deformation with their corresponding $a \in T_{X, x}=k^{n}$ (as in the beginning of (4.2)), then

$$
K_{j}=\left\{a \in T_{X, x} \mid a \cdot \nabla \varphi_{i} \in\left\{\begin{array}{ll}
g & \text { if } \nu_{i} \leqslant j, \\
g+\pi^{j+1} & \text { otherwise }
\end{array}\right\} .\right.
$$

$\left(\right.$ Recall $\theta_{X, x}=R / J, R=\theta_{A_{k, 0}^{n}}, M=\max (R), f_{1}, \ldots, f_{s}$ an $M$-standard base 
for $J, \nu_{i}=\nu_{M}\left(f_{i}\right), \varphi_{i}=\operatorname{In}_{M}\left(f_{i}\right), g=\operatorname{Gr}_{M}(J, R)$, $\Re$ the ideal of the vertex in $\operatorname{Gr}_{M}(R)$.)

(4.2.5) We have seen that $K_{j}$ may be identified with a subspace of $T_{X, x}$ via $\sigma \mapsto a$ as above. On the other hand, $K_{j}$ is the kernel of $T_{N_{j, x}} \rightarrow T_{S_{j, x}}$ (3.1). Therefore

$$
K_{j}=T_{N_{j, x}} \cap T_{\mathfrak{X}_{s_{0}, x}}
$$

where $s_{0}$ is the closed point of $S$. Hence, since $\mathfrak{X}_{s_{0}}=X$ we get another embedding $K_{j} \hookrightarrow T_{X, x}$. We want to check that these two embeddings are the same. To see this, let $\sigma \in K_{j} \subset \Re_{j}(D)=t_{\varkappa_{j}}$ (more precisely we should think of the element of $\varkappa_{j}(D)$ as the pair consisting of the trivial deformation over $D$ together with the section $\sigma$ ). Now since $t_{\Re_{j}}=T_{N_{j, x}}(1.2 .4) \sigma$ corresponds to a unique $\partial: D \rightarrow N_{j}$ :

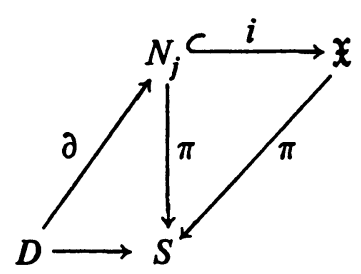

In particular the pullback via $\partial$ of

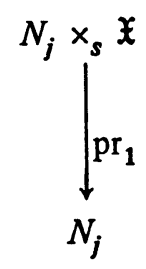

is the trivial deformation. This means that $\operatorname{Im}(i \cdot \partial) \hookrightarrow \mathfrak{X}_{s_{0}}$, and so the identification of $\sigma \in K_{j}$ with a tangent vector of $\mathfrak{X}_{s_{0}}$ at $x$ is made explicit: $\sigma \mapsto(i \cdot \partial)$. On the other hand, we know that $\sigma$ may be recovered as the pullback by $\partial$ of the versal section $\left(1_{N_{j}}, i\right): N_{j} \rightarrow N_{j} \times_{S} \mathfrak{X}$. Thus $\sigma$ is $\left(1_{D}, i \cdot \partial\right)$ : $D \rightarrow D \times_{k} \mathfrak{X}_{s_{0}}=D \times_{k} X$, so that if $\sigma$ corresponds to $a \in T_{X, x}$ (first embedding), $i \cdot \partial$ is given on coordinate rings by $x_{i} \mapsto \varepsilon a_{i}$, in other words, $i \cdot \partial$ is also the tangent vector $a$. We get the result: The functorial expression of either embedding is $\sigma \mapsto i \cdot \partial$. As a consequence,

(4.2.6) The subspace $K_{j} \subset T_{X, x}$ described explicitly in (4.2.4) is the tangent space of $N_{j} \cap X$ at $x$ (we view $X=\mathfrak{X}_{s_{0}}$ ). 


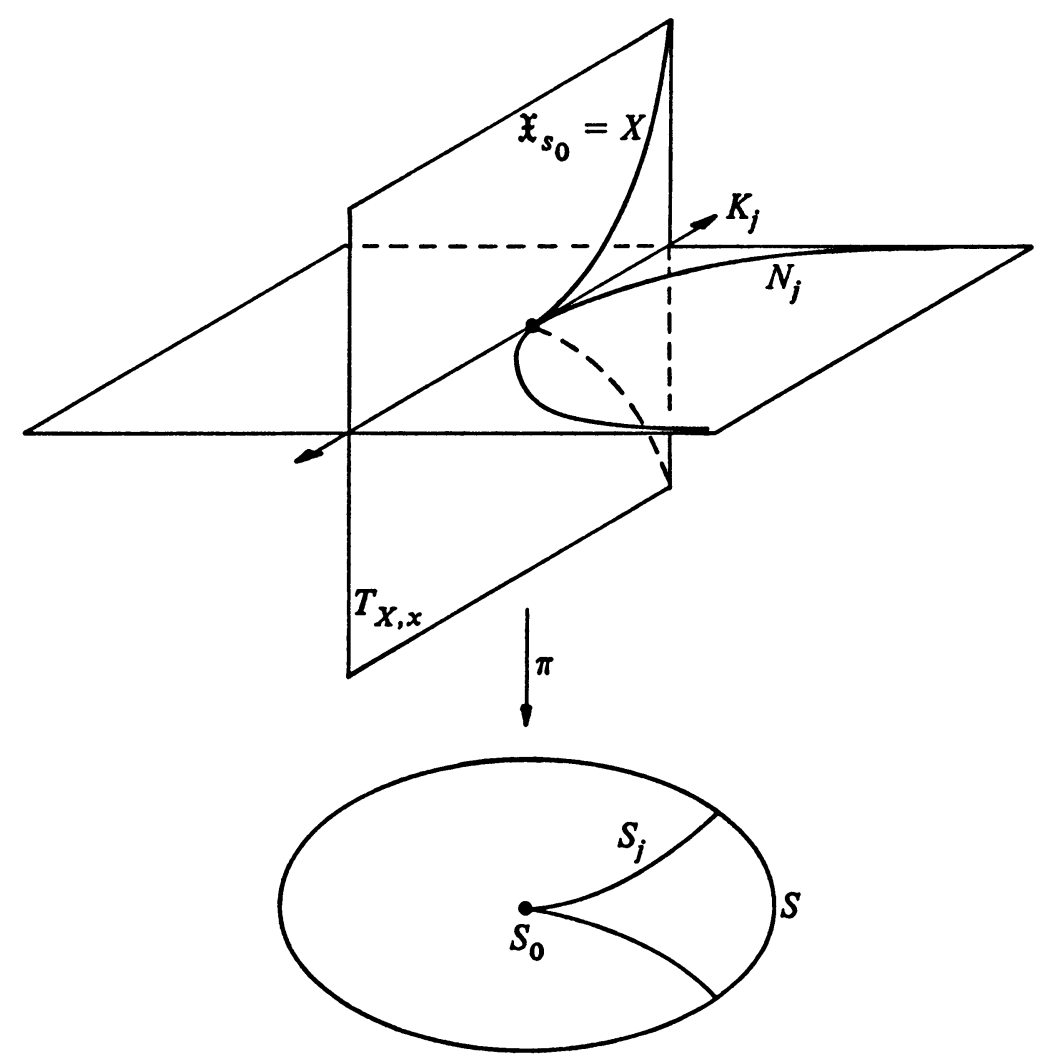

(In the above figure, the horizontal plane is included solely for spatial reference; only $X$ and $N_{j}$ are actually contained in $\mathfrak{X}$.)

\section{FIGURE (4.2.7)}

(4.2.8) REMARK. We note that $K_{0}$ is the space of all possible sections of the trivial deformation, so that $K_{0}=T_{X, x}$. On the other extreme, let $j$ be an integer $\geqslant \sup _{i}\left(\nu_{(x)}\left(f_{i}\right)\right)$, where the $f_{i}$ are an $(x)$-standard base of $J$ as usual. Then $\Re_{j+r}=\Re_{j}$ for all $r \geqslant 0$ (so the same is true of the $K$ s). Namely, normally flat of order $j$ means that each $f_{i}$ has been deformed without lowering $v_{i}$ (2.14). But then the family is normally flat (i.e. of all orders) by (2.13).

(4.3) Relationship of the $K_{j}$ with the tangent additive spaces. Let $X \hookrightarrow \mathrm{A}_{k}^{n}$ (in either the geometric or formal sense), and let $U$ be any subscheme of $A_{k}^{n}$. We can ask whether translation by $U$-in the sense of the additive vector group structure on $\mathrm{A}^{n}$-leaves $X$ invariant. More precisely, if $a: \mathrm{A}^{n} \times \mathrm{A}^{n} \rightarrow \mathrm{A}^{n}$ is the addition of vectors, we ask if the restriction of $a$ to $U \times X$ factors through $X$. More generally, let $X_{j}$ denote the $j$ th infinitesimal neighborhood of the origin $x \in X$; as usual, we think of $x$ as our distinguished singular point of $X$. Then, given $U$ as above, we ask if 
(*) $U$ leaves $X_{j}$ inside $X$, i.e. the restriction of $a$ to $U \times X_{j}$ factors through $X$. (This is of course much weaker than leaving $X_{j}$ invariant.)

(4.3.1) Given $X \hookrightarrow \mathbf{A}_{k}^{n}$, there is a maximum $U$ with the property (*), which we denote by $U_{j}(X, x)$, and which is a subscheme of $X$.

Proof. The map $a$ is given on rings by $z_{i} \mapsto z_{i}+u_{i}$, where we view $\mathbf{A}_{k}^{n} \times \mathbf{A}_{k}^{n}=\operatorname{Spec}\left(k\left[u_{1}, \ldots, u_{n}, z_{1}, \ldots, z_{n}\right]\right)$. If $X$ is defined by the ideal $J$ in $\mathbf{A}^{n}$, the condition that $U=\operatorname{Spec}\left(k\left[u_{1}, \ldots, u_{n}\right] / \mathfrak{X}\right)$ satisfies $(*)$ is then that

$$
f(z+u) \in\left(\mathfrak{A}+J+(z)^{j+1}\right) k[u, z] \text { for all } f \in J .
$$

Now choose a $k$-basis of $k[z] / J+(z)^{j+1}$ consisting, say, of monomials $z^{r}=z_{1}^{n}, \ldots, z_{n}^{r_{n}}$, as $r$ ranges over a certain finite set of multi-indices. We can think of $f(z+u)$ as the Taylor series

$$
f(z+u)=f(u)+z \cdot \nabla f(u)+\cdots,
$$

in which the coefficient of $z_{1}^{n}, \ldots, z_{n}^{r_{n}}$ is

$$
D_{r}(f(u))=1 / r ! \partial^{|r|} f(u) / \partial u_{1}^{n}, \ldots, \partial u_{n}^{r_{n}}
$$

where $|r|=r_{1}+\cdots+r_{n}, r !=r_{1} ! \cdots r_{n} !\left(^{12}\right)$. Then (4.3.2) is satisfied if and only if $D_{r}(f(u)) \in \mathfrak{A}$ for each $r$ and each $f$ in $J$. Hence the ideal generated by these $D_{r}(f(u))$ defines the largest $U$ with the desired property. Moreover, since the ideal in question contains $J$ (i.e. $\left.D_{0}(f(u))\right), U_{j}(X, x) \hookrightarrow X$.

(4.3.3) $U_{j}(X, x)$ represents the functor of $j$-sections of the trivial deformation of $X$, through $x$, which induce a trivial deformation of $X_{j}$. In fact, let $\tau$ denote the 0 -section of the projection $U_{j} x_{k} X_{j} \rightarrow U_{j}$ (where $U_{j}=U_{j}(X, x)$ ). By hypothesis, from the addition map we have $U_{j} \times X_{j} \stackrel{a}{\rightarrow} X$; thus we get an embedding $i=\left(\mathrm{pr}_{1}, a\right): U_{j} \times X_{j} \hookrightarrow U_{j} \times X$, which fits into a commutative diagram

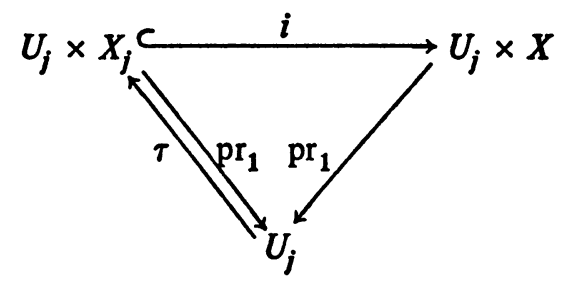

It is clear that $i \circ \tau$ gives a section of $U_{j} \times X$, and that the $j$ th infinitesimal neighborhood of this section is just the image of $i$ (and is therefore the trivial deformation of $X_{j}$ over $U_{j}$ ).

Now suppose, for some scheme $T$, we have a j-section $\sigma$ of $T \times_{k} X \rightarrow T$,

(12) In characteristic $p$ we take $D_{r} f(u)$ to mean the appropriate Hasse derivative, i.e. so that it will be the desired coefficient in the Taylor expansion. 
along which the $j$ th infinitesimal neighborhood varies trivially (and of course, $\sigma$ passes through $x$ in the special fibre). This means that we have a commutative diagram

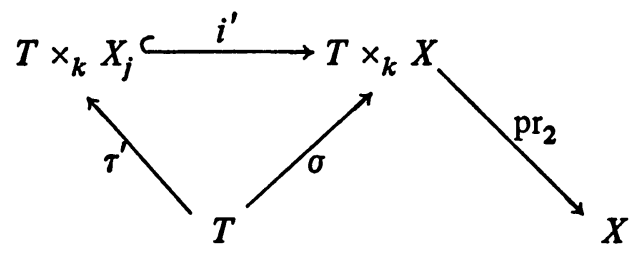

where $\tau^{\prime}$ is the 0 -section and $i^{\prime}$ is an embedding, which reduces to the embedding of $X_{j}$ in $X$ on the special fibre over $T$. Moreover, the composition $c=\mathrm{pr}_{2} \circ i^{\prime} \circ \tau^{\prime}$ maps $T$ into $X$, and hence into $\mathrm{A}_{k}^{n}$. It is then easy to check that $T \times_{k} X_{j} \stackrel{\mathrm{pr}_{2}{ }^{\circ}{ }^{\prime}}{\longrightarrow} X$ is induced by the addition in $A^{n}$, i.e. that the diagram

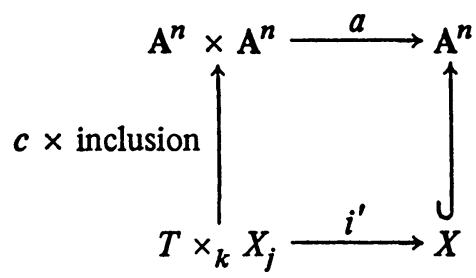

commutes; the result follows from the maximality of $U_{j}$. Moreover, we get

(4.3.4) The various $U_{j}(X, x)$ that are a priori associated to different embeddings of $X$ in affine space (of any dimension) are canonically isomorphic.

What is the relevance of this to our functors $\Re_{j}$, and in particular to our study of their tangent spaces? Let $\bar{N}_{j}$ denote the fibre of $N_{j}$ over the special point $s_{0}$ of $S$, so $\bar{N}_{j}=N_{j} \cap X$ where $X=\mathfrak{X}_{s_{0}}$, and $K_{j}=T_{\bar{N}_{j, x}}(4.2 .6)\left({ }^{3}\right)$. Then $\bar{N}_{j}$ represents the functor of $j$-sections through $x$ of the trivial deformation of $X$. This follows immediately from our basic set-up of (1.2), and is one way of expressing the fact that the map $N_{j} \rightarrow S$ induced by projection $\pi: \mathfrak{X} \rightarrow S$ represents the morphism of functors from $\Re_{j}$ to the ordinary deformation theory of $X$ which "forgets" the section. Thus it follows from (4.3.3) that

(4.3.5) $U_{j}(X, x)$ is canonically contained in $\bar{N}_{j}$.

However, it is easy to see that $U_{j}(X, x) \neq \bar{N}_{j}$ in general (even on the level of tangent spaces), or in other words, that a $j$-section of the trivial deformation does not necessarily induce a trivial deformation of $X_{j}$ (even to first order). Indeed, suppose $X$ is defined by an ideal $J$ in $\mathbf{A}_{k}^{n}$, and $f \in J$ with $\nu_{(x)}(f)=\nu$. Let $j \geqslant \nu$, and suppose that for some coordinate function $z$, the section $z=u, y_{1}=\cdots=y_{n-1}=0$ (where the $y i$ 's are the other coordinates on $\mathbf{A}^{n}$ )

(13) $N_{j}$ can also be described as the component through $x \in X$ of the simultaneous flat strata of the sheaves $P_{x / k}^{1}, \ldots, P_{x / k}^{j}$. 
of the trivial deformation over $k[u] / u^{2}$ is a $j$-section. Thus, if we change coordinates by using $z^{\prime}=z-u$ instead of $z$, the family is then given by an ideal $J^{\prime}$ of $k\left[u, z^{\prime}, y\right]$ which contains $f\left(z^{\prime}, y\right)+u \partial f / \partial z^{\prime}$, and the section is the 0 section. Assuming that $f$ is part of a standard base for $J$, the fact that $\sigma: z^{\prime}=y_{1}=\cdots=y_{n-1}=0$ is a $j$-section implies in particular that $\nu_{(x)} \partial f / \partial z^{\prime}$ $\geqslant v$; by suitable choice of $f$ we may also suppose that

$$
\partial f / \partial z^{\prime} \notin J^{\prime}+\left(z^{\prime}, y\right)^{j+1} .
$$

Now in order for the $j$ th infinitesimal neighborhood of $\sigma$ to be a trivial deformation of $X$, we must be able to eliminate $u$ from the generators of the ideal $J^{\prime}+\left(z^{\prime}, y\right)^{j+1}$ by a suitable change of coordinates. In view of $(\dagger)$, this change must be back to the original coordinates $(z, y)$. But this change must necessarily introduce $u$ as the coefficient of the derivative with respect to $z$ of any monomial of degree $j+1$. Thus, if we have chosen $J$ so that not all such derivatives are initial forms of elements of $J$, we see that the trivialization cannot be accomplished. For example, we may take $J=(f, g) k\left[z, y_{1}, y_{2}\right]$, where $f=y_{1}^{2}-z^{3}, g=y_{2}^{4}-z^{5}$, and $j \geqslant 2$.

However, it is easy to see that

(4.3.6) If $X: f=0$ is a hypersurface, with $\nu_{(x)}(f)=\nu$, then $U_{j}(X, x)=\bar{N}_{j}$ for $j \leqslant \nu-1$, i.e. for those $j$ which can give rise to distinct $N_{j}$ 's (recall that the $N_{j}$ 's stabilize after $j=\nu-1$ in the hypersurface case). Here $U_{j}=\bar{N}_{j}$ is the subscheme of $\mathbf{A}_{k}^{n}$ defined by the partial derivatives of $f$ of orders 0 through $j$.

As a corollary, we get

(4.3.7) If $X: f=0$ is a hypersurface with $\nu_{(x)}(f)=\nu$,

$$
K_{j}=T_{U_{j}(X, x), 0}
$$

for $j \leqslant \nu-1$, where 0 denotes the origin.

As we have seen, (4.3.7) is false for general singularities. However, we will find below that for arbitrary $X, x, K_{j}$ coincides with the tangent space at the origin of $U_{j}\left(C_{X, x}, 0\right)$ where $C_{X, x}$ is the tangent cone to $X$ at $x$.

(4.3.8) Definition. Let $X, x$ be an arbitrary singularity, and let $C_{X, x}$ denote its tangent cone. Then $U_{j}\left(C_{X, x}, 0\right)$ as called the jth tangent additive space of $X$ at $x$, and is denoted $A_{j, X, x}$ (or just $A_{j}$, if there is no danger of confusion).

(4.3.9) REMARK. We clearly have

$$
C_{X, x}=A_{0} \supset \cdots \supset A_{j} \supset A_{j+1} \supset \cdots \supset A=\bigcap_{j} A_{j},
$$

where $A$ is the largest subscheme of $A^{n}=T_{X, x}$ which leaves $C_{X, x}$ invariant by translation; $A$ is thus a subgroup-scheme of $T_{X, x}$ (this is false for $A_{j}$ when $j$ is small). In characteristic $0, A$ is a linear subspace of $A^{n}=T_{X, x}$, which can also be described as the largest linear subspace which splits off $C_{X, x}$, i.e. $C_{X, x}$ 
$=\left(C_{X, x} / A\right) \times_{k} A$. This linear subspace is sometimes called the strict tangent space to $X$ at $X$, denoted $T_{X, x}$. In characteristic $p, A$ involves finite subgroupschemes of $T_{X, x}$ which arise from $p$ th powers in the equations of this cone; this is related to the phenomena studied by Hironaka [12].

(4.3.10) THEOREM. For any $X, x$, and any $j \geqslant 0$,

$$
K_{j}=T_{A_{j, x, x}, 0}
$$

where 0 is the origin, and $K_{j}$ is the subspace of $t_{\sigma_{j, X, x}}$ introduced in (4.2), i.e. the space of $j$-sections through $x$ of the trivial first-order deformation of $X$.

Proof. Let $z_{1}, \ldots, z_{n}$ denote the coordinates on $A^{n}$, and let $D$ $=\operatorname{Spec}\left(k[\varepsilon] / \varepsilon^{2}\right)$. We will identify a tangent vector $\alpha=\left(\alpha_{1}, \ldots, \alpha_{n}\right)$ in $T_{A^{n}, 0}$ with the map $\alpha: D \rightarrow A^{n}$ defined by $z_{i} \mapsto \alpha_{i} \varepsilon$. Then $\alpha$ is in $T_{A_{j}, 0} \hookrightarrow T_{A^{n}, 0}$ if and only if the composition

$$
D \times_{k} C_{X, x, j} \stackrel{\alpha \times i}{\rightarrow} \mathrm{A}^{n} \times \times_{k} \mathrm{~A}^{n} \stackrel{a}{\rightarrow} \mathrm{A}^{n}
$$

factors through $C_{X, x}$ (where $C_{X, x, j}$ denotes the $j$ th infinitesimal neighborhood of the vertex in $C_{X, x}$, and $i: C_{X, x, j} \hookrightarrow \mathrm{A}^{n}$ is the inclusion; a denotes vector addition as usual). Now the image of this mapping is defined by the ideal

$$
\left\{\psi \in k[z] \mid \psi(z+\varepsilon \alpha) \subset g k[\varepsilon, z]+(z)^{j+1} k[\varepsilon, z]\right\}
$$

where $z=\left(z_{1}, \ldots, z_{n}\right), \alpha=\left(\alpha_{1}, \ldots, \alpha_{n}\right)$ and $g$ is the ideal of $C_{X, x}$ in $\mathbf{A}^{n}$. Hence $\alpha \in T_{A_{j}, 0}$ if and only if $g$ itself is in this ideal, i.e.

$$
\varphi_{i}(z+\varepsilon \alpha) \in g k[\varepsilon, z]+(z)^{j+1} k[\varepsilon, z]
$$

where the $\varphi_{i}$ generate $g_{\text {; }}$ we may assume for example that the $\varphi_{i}$ are the initial forms of the elements of an $(x)$-standard base for $J$, where $J$ is the ideal of $X$ in $A^{n}$. Noting that $\varphi_{i}(z+\varepsilon \alpha)=\varphi_{i}+\varepsilon \alpha \cdot \nabla \varphi_{i}$, the condition above is equivalent to $\alpha \cdot \nabla \varphi_{i} \in g+(z)^{j+1}$ for each $i$. Now for those $i$ such that $\nu_{i}=\operatorname{deg} \varphi_{i}$ $\leqslant j, \operatorname{deg}\left(\alpha \cdot \nabla \varphi_{i}\right)=\nu_{i}-1<j$, so that for these $i, \alpha \cdot \nabla \varphi_{i} \in g+(z)^{j+1} \Leftrightarrow \alpha$ - $\nabla \varphi_{i} \in g$. We get:

$$
T_{A_{j}, 0}=\left\{\alpha \in T_{A^{n}, 0} \mid \alpha \cdot \nabla \varphi_{i} \in\left\{\begin{array}{ll}
g & \text { if } \nu_{i} \leqslant j, \\
g+(z)^{j+1} & \text { otherwise }
\end{array}\right\},\right.
$$

and the result follows from (4.2.4).

(4.3.11) REMARK. Even though $K_{j}=T_{\bar{N}, x}$ agrees with $T_{A_{j, 0}}$ for any $X, x$, it is not true that $\bar{N}_{j}=A_{j, X, x}$; in fact when $X$ is an isolated singularity $\bar{N}_{j}$ is always artinian, but $A_{j}$ need not be $(j>0)$. When $X$ is a cone, however, (with or without isolated singularities) and $x$ is the vertex, then one can show 
$\bar{N}_{j}=A_{j}$; this case, together with the hypersurface case (4.3.6) are essentially the only situations where this is true. A study of the higher order structure of the $\bar{N}_{j}$ for general singularities will be the subject of a sequel to this work; even in the hypersurface case much more can be said. At any rate, it is shown in [14] that $N_{j}$ is the normalization of its image in $S_{j}$ when $X$ is a strict complete intersection at $x$ in characteristic 0 , and in characteristic $p$ is a purely inseparable cover thereof.

(4.4) The image of $\delta$. Up to now we have been concerned with the kernel $K_{j}$ of the map

$$
\delta: t_{\Im,}=T_{N_{j, x}} \rightarrow T_{S, s_{0}}=t_{F}
$$

of tangent spaces induced by

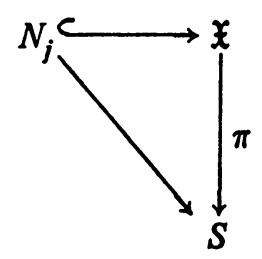

where $S$ is the space of formal moduli of $X$, i.e. the prorepresentable hull of the ordinary deformation functor $F$ of $X$, and $\mathfrak{X} / S$ is the versal formal deformation with special fibre $X=\mathfrak{X}_{s_{0}}$. We have seen that $K_{j}$ can be interpreted either as the space of $j$-sections of the trivial first-order deformation of $X$, or as the first-order " $j$ th tangent additive space" to $X$ at $x$ (4.3). We now want to study the image of $\delta$ in $T_{S, s_{0}}$. (Of course, we can view the image as lying in $T_{S_{j}, s_{0}}$, where $S_{j}$ is the image of $N_{j}$ by $\pi_{j}$; however this will not be so important in the present content.)

The image of $\delta$ can be described in terms of a certain filtration of $T_{S, s_{0}}$. Indeed, although we think of $T_{S, s_{0}}$ as a vector space, it also has a canonical $\theta$ module structure, where $\theta=\theta_{X, x}$. In fact, let $\theta=R / J$, where $R$ is the local ring at $x$ in some smooth space $W$ over $k$ in which $X$ is embedded (locally at $x$ ). Then, according to the theory of Schlessinger we have an exact sequence of $\theta$-modules which gives an explicit realization of $T_{S, s_{0}}$ :

$$
0 \rightarrow \operatorname{Der}_{k}(\theta, \theta) \rightarrow \operatorname{Der}_{k}(R, \theta) \rightarrow \operatorname{Hom}_{\Theta}\left(J / J^{2}, \theta\right) \rightarrow T_{S, s_{0}} \rightarrow 0
$$

which comes from dualizing the exact sequence of differentials $\mathrm{J} / \mathrm{J}^{2} \rightarrow \Omega_{R / k}^{1}$ $\otimes_{k} \theta \rightarrow \Omega_{\theta / k}^{1} \rightarrow 0$. Equivalently, in the language of the cotangent complex of Schlessinger-Lichtenbaum [5], [13] the sequence (4.4.1) may be written

$$
0 \rightarrow T_{X, x}^{0} \rightarrow i^{*} T_{W, x}^{0} \rightarrow N_{X / W, x} \rightarrow T_{X, x}^{1} \rightarrow 0
$$


where $i: X \hookrightarrow W$ is the inclusion, and $N_{X / W}$ is the normal sheaf to $X$ in $W$. Thus $T_{S, s_{0}}, t_{F}, T_{X, x}^{1}$ all stand for the same object. In this section, where we wish to stress the $\theta$-module structure, we will henceforth use the notation $T_{X, x}^{1}$, or just $T^{1}$ for short, instead of $T_{S, s_{0}}$ or $t_{F}$ We have, from (4.4.1):

$$
T^{1}=\left(J / J^{2}\right)^{*} / \Delta
$$

where ${ }^{*}$ denotes $\theta$-dual, and $\Delta$ is the submodule consisting of those homomorphisms, which, for some $k$ derivation $D$ of $R$ to itself, have the form $f\left(\bmod J^{2}\right) \mapsto D(f)(\bmod J)$.

Now we describe the filtration: Let $f_{1}, \ldots, f_{r}$ be an $(x)$-standard base of $J$ (i.e. an $M$-standard base where $M=\max (R)$ ), and let $\nu_{i}=\nu_{M}\left(f_{i}\right)$. Suppose the $f_{i}$ are arranged so that $\nu_{1} \leqslant \nu_{2} \leqslant \cdots \leqslant \nu_{r}$; for any positive integer $j$ we let $i(j)$ denote the largest index such that $\nu_{i(j)} \leqslant j$. Let $F=\theta^{r}$ denote the free $\theta$ module of rank $r$, with the standard basis $e_{1}, \ldots, e_{r}$, and let $\Re=\max (\theta)$. We filter $F^{*}=\operatorname{Hom}_{\theta}(F, \theta)$ by letting

$$
F_{j}^{*}=\text { The submodule consisting of those homomorphisms } \alpha
$$$$
\text { for which } \alpha\left(e_{i}\right) \in \mathscr{N}^{\nu_{i}} \text { for } i \leqslant i(j) \text {, and } \alpha\left(e_{i}\right) \in \mathscr{N}^{j+1}, i>i(j) \text {. }
$$

Equivalently, if we identify $F^{*}$ with $\theta^{r}$ via the dual basis $e_{1}^{*}, \ldots, e_{r}^{*}$, then

$$
F_{j}^{*}=\pi^{\nu_{1}} \oplus \cdots \oplus \Re^{\nu_{i}(j)} \oplus \Re^{j+1} \oplus \cdots \oplus \Re^{j+1} \subset \boldsymbol{\theta}^{r} .
$$

Note that this filtration is not exhaustive, in fact (in the sense $n \mathfrak{f}(4.4 .3)$ ), for $j \geqslant \sup \left(v_{i}\right)$

$$
F_{j}^{*}=\Re^{\nu_{1}} \oplus \cdots \oplus \mathfrak{N}^{\nu_{r}} .
$$

Now $F$ maps surjectively onto $J / J^{2}$ via $e_{i} \mapsto$ class of $f_{i}$, and hence we get an injection $\left(J / J^{2}\right)^{*} \hookrightarrow F^{*}$. The filtration described above on $F^{*}$ induces one on $\left(J / J^{2}\right)^{*}$, i.e.

$$
\left(J / J^{2}\right)_{j}^{*}=_{\text {def }}\left(J / J^{2}\right)^{*} \cap F_{j}^{*} .
$$

(4.4.4) Definition. $T_{j}^{1}$ is the image of $\left(J / J^{2}\right)_{j}^{*}$ in $T^{1}$ (in the sense of (4.4.2)). That this definition is independent of the embedding $\theta=R / J$, of the choice of standard base is a corollary of the theorem below:

(4.4.5) THEOREM. $T_{j}^{1}$ is the image of $T_{N_{j}, x}$ in $T^{1}=T_{S, s_{0}}$ by $\delta$.

Proof. The image of $T_{N_{j}, x}$ in $T^{1}$ is the space of first-order deformations of $X$, say over $k[\varepsilon] / \varepsilon^{1}$, which admit a $j$-section. If $Z$ is such, with $j$-section say $z_{1}=\cdots=z_{n}=0$, then in terms of our standard base $f_{1}, \ldots, f_{r}$ it is given by 
equations $f_{i}(z)+\varepsilon g_{i}(z), i=1, \ldots, r$, where we view the $g_{i}$ as being in $R$; by our criterion (2.14) we may assume that they satisfy: $\nu_{M}\left(g_{i}\right) \geqslant \nu_{i}$ for $i \leqslant i(j)$, and $\nu_{M}\left(g_{i}\right)>j$ for $i>i(j)$. Moreover, since $Z$ is flat over $k[\varepsilon] / \varepsilon^{2}$, we know that the $g_{i}$ are representatives in $R$ of elements $\alpha\left(\bar{f}_{i}\right) \in \mathcal{O}$, where $\bar{f}_{i}$ denotes the class of $f_{i}$ in $J / J^{2}$, and $\alpha \in\left(J / J^{2}\right)^{*}$. Now the conditions above on the $\nu_{(z)}\left(g_{i}(z)\right)$ imply a fortiori that $\nu_{\mathscr{N}}\left(\alpha\left(\bar{f}_{i}\right)\right) \geqslant \nu_{i}$ if $i \leqslant i(j)$, and $\nu_{\mathscr{N}}\left(\alpha\left(\bar{f}_{i}\right)\right)>j$ otherwise. We have shown, therefore, that the image of $\delta$ is contained in $T_{j}^{1}$.

Conversely, suppose a deformation $Z$ over $k[\varepsilon] / \varepsilon^{2}$ is in $T_{j}^{1} . Z$ is defined by equations $f_{i}(z)+\varepsilon g_{i}(z), i=1, \ldots, r$, in $R[\varepsilon] / \varepsilon^{2}$ (where now $z_{1}, \ldots, z_{n}$ denote a regular system of parameters of $R$ ). Moreover, if $\bar{g}_{i}$ is the image of $g_{i}$ in $\theta$, then by definition of $T_{j}^{1},\left(\bar{g}_{1}, \ldots, \bar{g}_{r}\right)$ is in $\mathfrak{N}^{\nu_{1}} \oplus \cdots \oplus \mathfrak{N}^{v_{i}(j)} \oplus \mathfrak{N}^{j+1} \oplus \cdots$ $\oplus \Re^{j+1}$ modulo the submodule generated by $\left(\overline{\partial f_{1} / \partial z_{l}}, \ldots, \overline{\partial f_{r} / \partial z_{l}}\right), l=1$, $\ldots, n$. This means that for some choice of

$$
\begin{gathered}
\left(h_{1}, \ldots, h_{n}\right) \in R^{n}, \quad\left(s_{1}, \ldots, s_{r}\right) \in J^{r}, \\
\left(\tilde{g}_{1}, \ldots, \tilde{g}_{r}\right) \in M^{v_{1}} \oplus \cdots \oplus M^{\nu_{i}(j)} \oplus M^{j+1} \oplus \cdots \oplus M^{j+1},
\end{gathered}
$$

we have

$$
\left(g_{1}, \ldots, g_{r}\right)=\left(\tilde{g}_{1}+\sum_{l=1}^{n} h_{l} \frac{\partial f_{1}}{\partial z_{l}}+s_{1}, \ldots, \tilde{g}_{r}+\sum_{l=1}^{n} h_{l} \frac{\partial f_{r}}{\partial z_{l}}+s_{r}\right)
$$

Now we want to show that the deformation $Z$ admits a j-section); in fact we claim that $z_{l}=-\varepsilon h_{l}, l=1, \ldots, n$, is such a section. To see this, let $\tilde{z}_{l}$ $=z_{l}+\varepsilon h_{l}$ (so the section is $\left.\sigma: z_{l}=0, l=1, \ldots, n\right)$. Then in terms of the coordinates $\tilde{z}_{l}$, for each $i$ :

$$
\begin{aligned}
f_{i}(z)+\varepsilon g_{i}(z) & =f_{i}(\tilde{z}-\varepsilon h)+\varepsilon g_{i}(\tilde{z}-\varepsilon h) \\
& =f_{i}(\tilde{z})+\varepsilon\left(-\sum_{l=1}^{n} h_{l}(\tilde{z}) \frac{\partial f_{i}(\tilde{z})}{\partial \tilde{z}_{l}}+g_{i}(\tilde{z})\right) .
\end{aligned}
$$

Now by (4.4.6) the expression in parentheses is just $\tilde{g}_{i}(\tilde{z})+s_{i}$, so we get: For each $i=1, \ldots, r, f_{i}(z)+\varepsilon g_{i}(z)=f_{i}(\tilde{z})+\varepsilon \tilde{g}_{i}(\tilde{z})+\varepsilon s_{i}$, with $\nu_{(\tilde{z})}\left(\tilde{g}_{i}(\tilde{z})\right)$ $\geqslant \nu_{i}$ for $i \leqslant i(j)$, and $\nu_{(\bar{z})}\left(\tilde{g}_{i}(\tilde{z})\right)>j$ otherwise. However, since $s_{i}$ is in $J$ $=\left(f_{1}, \ldots, f_{r}\right) R$, and $\varepsilon^{2}=0$, it is easy to check that the ideal generated by the $f_{i}+\varepsilon \tilde{g}_{i}+\varepsilon s_{i}$ is the same as that generated simply by the $f_{i}+\varepsilon \tilde{g}_{i}$. Hence we conclude from (2.14) that $\sigma$ is a $j$-section. Q.E.D.

(4.4.7) REMARK. Since the image of $\delta$ is functorial, it follows that the filtration $T_{j}^{1}$ is intrinsic, and is independent of any choice of embedding or standard base.

(4.4.8) Example. If $X: f=0$ is a hypersurface, $T^{1}$ can be identified with $k[[z]] /\left(f, \partial f / \partial z_{i}\right)$, where we view $x=$ origin. Suppose $\nu_{(z)}(f)=\nu$. If $j \leqslant \nu$ -1 , then $T_{j}^{1}$ is just $(z)^{j+1} k[[z]] /\left(f, \partial f / \partial z_{i}\right)$. If $j \geqslant \nu-1, T_{j}^{1}$ is 


$$
(z)^{\nu} k[[z]] /\left(f, \partial f / \partial z_{i}\right) \text {. }
$$

The following theorem summarizes the main results of $\S 4$ :

(4.4.9) Theorem. Let $\varkappa_{j}$ denote, as usual, the functor of normally flat deformations of order $j$ of $X$ at $x$, and let $F$ denote the ordinary deformation functor of $X$. Let $\delta: t_{\vartheta_{j}} \rightarrow t_{F}$ be the map on tangent spaces induced by the morphism of functors which forgets the section. Then (noncanonically)

$$
t_{\Re_{j}} \cong K_{j} \oplus T_{j}^{1}
$$

where $K_{j}$ may be canonically identified with

(i) $\operatorname{ker} \delta$,

(ii) the space of $j$-sections through $x$ of the trivial first-order deformation of $X$ ((4.3.4) ff.),

(iii) the tangent space at the origin to the " $j$ th tangent additive space $\mathbf{A}_{j, X, x}$ of $X$ at $x$ " (4.3.10),

And $T_{j}^{1}$ is

(i) the image of $\delta$ in $T_{F}\left(=T^{1}\right.$ in the notation of Schlessinger),

(ii) the jth piece in a certain filtration of $T^{1}$, induced by the $\Re$-adic one (via the canonical structure of $T^{1}$ as $\theta$-module (4.4.5); here $\theta=\theta_{X, x}, \Re=\max (\theta)$.

REMARK. It is interesting to inquire whether the splitíing in (4.4.10) is in any sense canonical. This is equivalent to asking whether each element of $T_{j}^{1}$ (viewed as a first order deformation of $X$ ) has a canonical $j$-section. Note that for the proof of (4.4.5) we only have to produce some $j$-section.

\section{BIBLIOGRAPHY}

1. A. Grothendieck and J. Dieudonné, Éléments de géométrie algébrique, Inst. Hautes Études Sci. Publ. Math. No. 32 (1967). MR 39 \#220.

2. D. Mumford, Lectures on curves on an algebraic surface, Ann. of Math. Studies, no. 59, Princeton Univ. Press, Princeton, N. J., 1966. MR 35 \# 187.

3. - Further pathologies in algebraic geometry, Amer. J. Math. 84 (1962), 642-648. MR 26 \#6177.

4. M. Schlessinger, Functors of Artin rings, Trans. Amer. Math. Soc. 130 (1968), 208-222. MR 36 \# 184.

5. - Thesis, Harvard Univ., Cambridge, Mass., 1964.

6. B. M. Bennett, On the characteristic functions of a local ring, Ann. of Math. (2) 91 (1970), 25-87. MR 40 \#5608.

7. M. Lejeune-Jalabert and B. Teissier, Normal cones and sheaves of relative jets, Thèses, Ecole Polytechnique.

8. H. Hironaka, Resolution of singularities of an algebraic variety over a field of characteristic $\mathbf{0 .}$ I, II, Ann. of Math. (2) 79 (1964), 109-203, 205-326. MR 33 \# 7333.

9. B. Teissier, Deformations d type topologique constant. I, II, Séminaire Douady-Verdier, Secrétariat Mathématique, Paris, 1972.

10. J. Wahl, Deformations of branched covers and equisingularity, Thesis, Harvard Univ., Cambridge, Mass., 1971.

11. F. Pham, Classification des singularités, Université de Nice (mimeographed notes). 
12. H. Hironaka, Additive groups associated with points of a projective space, Ann. of Math. (2) 92 (1970), 327-334. MR 42 \#4553.

13. S. Lichtenbaum and $M$. Schlessinger, The cotangent complex of a morphism, Trans. Amer. Math. Soc. 128 (1967), 41-70. MR 35 \#237.

14. B. Bennett, Normalization theorems for certain modular discriminantal loci, Compositio Math. 32 (1976), fasc. 1, 13-32.

15. J. Giraud, Sur la théorie du contact maximal, Math. Z. 137 (1974), 285-310.

16. - Contact maximal en caracteristique positive (preprint).

Department of Mathematics, Stanford University, Stanford, California 94305 